\title{
Influência do comportamento marginal na densidade conjunta bivariada
}

\author{
Mariela Fernández
}

TESE APRESENTADA

$\mathrm{AO}$

INSTITUTO DE MATEMÁTICA E ESTATÍSTICA

DA

UNIVERSIDADE DE SÃO PAULO

PARA

OBTENÇÃO DO GRAU

DE

DOUTOR EM CIÊNCIAS

Área de Concentração: Matemática Aplicada

Orientador: Prof. Dr. Nikolai Valtchev Kolev

Durante a elaboração deste trabalho a autora recebeu apoio financeiro do CNPQ

São Paulo - junho - 2007 



\title{
Influência do comportamento marginal na densidade conjunta bivariada
}

\author{
Este exemplar corresponde à redação final da tese \\ devidamente corrigida e defendida por Mariela \\ Fernández e aprovada pela Comissão Julgadora.
}

São Paulo, julho de 2007.

Banca examinadora:

- Prof. Dr. Nikolai Valtchev Kolev (Orientador) - IME-USP

- Prof. Dr. Henrique Von Dreifus - IME-USP

- Prof. Dr. Francisco Louzada Neto - UFSCar

- Prof. Dr. Dani Gamerman - UFRJ

- Prof. Dr. Cristiano Fernandes - PUC-RIO 

Ao Leonardo.

"As mais lindas frases de amor são ditas no silêncio de um olhar." 



\section{Agradecimentos}

A minha família, principalmente a minha mãe, avó e irmão porque sem eles não teria atingido nenhum dos meus objetivos. Também fico muito grata ao Joaquín, meu primeiro sobrinho e afilhado, saber que ele viria ao mundo foi uma grande motivação para continuar.

Ao Leonardo por ser um ótimo companheiro e tornar a minha fase de doutorando e a minha vida mais agradável.

Aos meus colegas e amigos Silvina, Florencia, Julieta, Cristian, Sandra e Marcelo.

Ao professor Nikolai Kolev por sua orientação.

À banca examinadora pelas sugestões expostas. Em especial ao professor Cristiano Fernandes pelos aprimoramentos sugeridos tanto no exame de qualificação quanto na defesa da tese. 



\title{
Resumo
}

Neste trabalho propomos a representação de uma densidade bivariada segundo a natureza geométrica das marginais. Uma densidade contínua pode ser aproximada pela exponencial de um polinômio num domínio limitado. Tal aproximação permite estudar a influência do comportamento marginal e condicional sobre a densidade conjunta. Esse estudo é realizado encontrando os conjuntos dos possíveis valores dos coeficientes do polinômio segundo a informação dada sobre as densidades marginais ou as densidades condicionais. Também analisamos os coeficientes segundo algumas medidas de dependência. Concluímos mostrando a influência do comportamento marginal e condicional sobre variáveis aleatórias discretas.

\begin{abstract}
In this work we propose a representation of a bivariate density according to the geometrical nature of the marginals. A continuous density can be approximated by the exponencial of a polynomial in a finite domain. Such approximation let us study the influence of the marginal and conditional behavior on the joint density. This study is done by finding the possible values of the polynomial coefficients upon the given information about the marginal or the conditional densities. We also analyze the coefficients according some dependence measures. We conclude showing the influence of the marginal and conditional behavior on discrete random variables.
\end{abstract}





\section{Índice}

$\begin{array}{lr}\text { Tabela de notação } & 3\end{array}$

1 Introdução $\quad 5$

2 Aproximação de uma densidade pela exponencial de um polinômio $\quad 7$

2.1 Domínio limitado . . . . . . . . . . . . . . . . . . . . . . . 7

2.2 Domínio ilimitado . . . . . . . . . . . . . . . . . . . . . . . . 10

3 Comportamento geométrico das densidades marginais $\quad 18$

3.1 Polinômio de grau um . . . . . . . . . . . . . . . . . . . . . . . . 19

3.2 Polinômio de grau dois . . . . . . . . . . . . . . . . . . 20

3.2.1 Família de três parâmetros . . . . . . . . . . . . . . . . . 20

3.2 .2 Família de quatro parâmetros . . . . . . . . . . . . . . . 30

3.2 .3 Família de cinco parâmetros . . . . . . . . . . . . . . . . . . . 37

3.3 Polinômio de grau três . . . . . . . . . . . . . . . . . . . . . . . 40

4 Comportamento geométrico das densidades condicionais 47

4.1 Polinômio de grau dois . . . . . . . . . . . . . . . . . . . . . 47

4.1 .1 Família de três parâmetros . . . . . . . . . . . . . . . . 47

4.1 .2 Família de quatro parâmetros . . . . . . . . . . . . . . . . 54

$4.1 .3 \quad$ Família de cinco parâmetros . . . . . . . . . . . . . . . . . . . 59

4.2 Polinômio de grau três $\ldots \ldots \ldots$. . . . . . . . . . . . . . 61 
5 Medidas de dependência $\quad 66$

5.1 Função de dependência local . . . . . . . . . . . . . . . . . . . . . . . 66

5.2 Dependência positiva . . . . . . . . . . . . . . . . . . 69

5.3 Dependência negativa . . . . . . . . . . . . . . . . . . . . . 72

6 Variáveis discretas $\quad 76$

6.1 Tabelas $2 \times 2 \ldots \ldots \ldots \ldots \ldots$

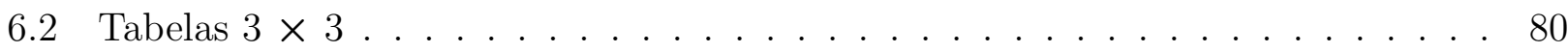

7 Conclusões e estudos futuros $\quad 86$

$\begin{array}{ll}\text { Referências Bibliográficas } & 90\end{array}$

$\begin{array}{ll}\text { A Demonstrações de resultados auxiliares } & 91\end{array}$

$\begin{array}{lll}\text { B Demonstrações dos resultados do capítulo } 2 & 98\end{array}$

$\begin{array}{lll}\text { C } & \text { Demonstrações dos resultados do capítulo } 3 & 108\end{array}$

D Demonstrações dos resultados do capítulo $4 \quad 128$ 


\section{Tabela de notação}

$[a, b]$ Intervalo fechado de extremo inferior $a$ e superior $b$.

]$a, b[\quad$ Intervalo aberto de extremo inferior $a$ e superior $b$.

$\gamma_{h} \quad$ Função de dependência local dada na equação (5.1).

$\psi \quad$ Função definida na equação (3.3).

AN Associação negativa, definição 5.3.3.

AP Associação positiva, definição 5.2.3.

$f_{X} \quad$ Função de densidade da variável $X$.

$f_{X}^{c} \quad$ Função marginal $f_{X}$ constante.

$f_{X}^{d} \quad$ Função marginal $f_{X}$ decrescente.

$f_{X}^{i} \quad$ Função marginal $f_{X}$ crescente.

$f_{X}^{m} \quad$ Função marginal $f_{X}$ com um ponto mínimo.

$f d l$ Função de dependência local dada na equação (5.1).

$g_{Y} \quad$ Função de densidade da variável $Y$.

$g_{Y}^{c} \quad$ Função marginal $g_{Y}$ constante.

$g_{Y}^{d} \quad$ Função marginal $g_{Y}$ decrescente.

$g_{Y}^{i} \quad$ Função marginal $g_{Y}$ crescente.

$g_{Y}^{m} \quad$ Função marginal $g_{Y}$ com um ponto mínimo.

$h \quad$ Função de densidade conjunta.

$K \quad$ Função auxiliar definida na Proposição A.1.

$L \quad$ Função definida na equação (3.6).

LTD Decrescente na cauda esquerda, definição 5.2.2.

LTI Crescente na cauda esquerda, definição 5.3.5.

$\mathbb{N} \quad$ Conjunto dos números naturais.

NDQ Negativamente dependente em quadrante, definição 5.3.1. 


\section{Tabela de notação (continuação)}

$P_{f} \quad$ Polinômio definido por $P_{f}(x, y)=\lambda_{10}+\lambda_{11} y+2 \lambda_{20} x+2 \lambda_{21} x y$.

$P_{g} \quad$ Polinômio definido por $P_{g}(x, y)=\lambda_{01}+\lambda_{11} x+2 \lambda_{02} y+\lambda_{21} x^{2}$.

$p_{i} \bullet \quad$ Probabilidade $\mathbb{P}(X=i)$, sendo $X$ uma variável aleatória discreta.

$p_{\bullet j} \quad$ Probabilidade $\mathbb{P}(Y=j)$, sendo $Y$ uma variável aleatória discreta.

$p_{i j} \quad$ Probabilidade $\mathbb{P}(X=i, Y=j)$, sendo $X$ e $Y$ variáveis aleatórias discretas.

PQD Positivamente dependente em quadrante, definição 5.2.1.

$\mathbb{R} \quad$ Conjunto dos números reais.

RTD Decrescente na cauda direita, definição 5.3.5.

RTI Crescente na cauda direita, definição 5.2.2.

SD Estocasticamente decrescente, definição 5.3.5.

sgn Função sinal definida na equação (3.7).

SI Estocasticamente crescente, definição 5.2.2.

TP Densidade totalmente positiva, definição 5.2.4.

TN Densidade totalmente negativa, definição 5.3.7.

$y_{a} \quad$ Ponto definido em (4.4).

$y_{b} \quad$ Ponto definido em (4.5). 


\section{Capítulo 1}

\section{Introdução}

$\mathrm{Na}$ grande maioria das análises estatísticas aplicadas necessitamos modelar populações multidimensionais. Usualmente conhecemos mais e melhor os processos marginais do que a estrutura de dependência. Por isso é comum na prática preferir implementar os modelos marginais em sistemas de informação e usá-los para realizar a análise de modelos multivaridos mais complexos. Levando em conta tal tecnologia, no presente trabalho propomos encontrar as possíveis formas de uma densidade bivariada assumindo o conhecimento das marginais. A necessidade de construir distribuições multivariadas supondo que as marginais são dadas surge com muita frequiência em problemas de diversas índoles. Dada a importância deste tema em aspectos tanto de pesquisa quanto de aplicação, uma série de conferências denominadas "Distributions with Given Marginals" foram organizadas em Roma (1990), Seattle (1993), Praga (1996), Barcelona (2000), Quebec (2004) e Tartu (2007). Em Cuadras et al (2002) pode ser vista uma resenha desses eventos. Como outro exemplo citamos o trabalho de Kotz e van Dorp (2002) no qual estudaram a família Fairlie-Gumbel-Morgenstern considerando que as distribuições marginais são do tipo "Two-Sided Power". Aqui sugerimos uma classificação de densidades bivariadas segundo o comportamento crescente ou decrescente das correspondentes marginais.

Existe muita bibliografia em relação a análise ou construção de densidades bivariadas via expansão em séries, porém a maioria dos trabalhos utiliza a informação de medidas de dependência como os momentos. Como exemplos temos as expansões de Gram-Charlier e de Edgeworth; ver Johnsons e Kotz (1972), e a expansão em variáveis canônicas ou autofunções 
dada por Lancaster (1958). Também é um outro exemplo a solução do Problema dos Momentos de Hausdorff; ver Shohat e Tamarkin (1943), Knill (1997) e Ang et al (1999). No nosso estudo também trabalhamos com expansão em séries da densidade conjunta mas determinada pela informação do comportamento marginal. Uma densidade contínua pode ser representada como segue

$$
h(x, y)=\exp \left(\sum_{i, j=0}^{\infty} \lambda_{i j} x^{i} y^{j}\right)
$$

sendo $\lambda_{i j}(i, j=0,1, \cdots)$ coeficientes reais e a igualdade como limite uniforme (a prova é apresentada no capítulo 2). Truncando esta última relação, e tomando em conta o conhecimento da natureza das marginais, pretendemos determinar uma possível expressão para a densidade conjunta $h(x, y)$. Especificamente, para as densidades

$$
h(x, y)=\exp \left(\lambda_{0}+\lambda_{10} x+\lambda_{01} y+\lambda_{11} x y+\lambda_{20} x^{2}+\lambda_{02} y^{2}\right), \quad(x, y) \in[a, b] \times[a, b]
$$

e

$$
h(x, y)=\exp \left(\lambda_{0}+\lambda_{10} x+\lambda_{01} y+\lambda_{11} x y+\lambda_{21} x^{2} y\right), \quad(x, y) \in[a, b] \times[a, b]
$$

sendo $[a, b] \subset \mathbb{R}$, determinamos os possíveis valores dos coeficientes $\lambda_{i j}, i, j=0,1,2$, segundo o comportamento geométrico das marginais (por exemplo funções crescentes, decrescentes, unimodais). Observemos que cada elemento da série, ou seja, cada expressão exp $\left(\sum_{i, j=0}^{n} \lambda_{i j} x^{i} y^{j}\right)$ é um elemento de uma família de exponenciais. Tal família é muito utilizada na prática pelas propriedades estatísticas que possui. No presente trabalho não expomos detalhes sobre a família de exponenciais. Estas podem ser consultadas, por exemplo, em Barndorff-Nielsen (1978) e em Nguyen e Rogers (1989).

No capítulo 2 apresentamos a demonstração da aproximação de uma densidade contínua por uma seqüência de densidades da forma de exponencias de polinômios. Continuamos, no capítulo 3, analisando como o comportamento geométrico das marginais influencia nos coeficientes de duas aproximações específicas. Uma vez concluída essa análise, passamos ao capítulo 4 onde aplicamos a metodologia anterior para o caso em que conhecemos a natureza geométrica de uma das densidades condicionais. No capítulo 5 obtemos as expressões de algumas medidas de dependência para as aproximações estudadas. Finalmente, no capítulo 6, exemplificamos como pode ser aplicada a ferramente aqui proposta para o caso de variáveis discretas. 


\section{Capítulo 2}

\section{Aproximação de uma densidade pela exponencial de um polinômio}

Começamos o presente trabalho mostrando o resultado no qual se baseia o nosso estudo. Especificamente, provamos que uma densidade pode ser aproximada pela exponencial de um polinômio num domínio limitado.

Primeiramente, na seção 2.1, estudamos o caso em que a densidade conjunta não é zero num conjunto limitado. Como segundo passo, na seção 2.2 , provamos a aproximação quando a densidade é positiva num conjunto ilimitado.

\subsection{Domínio limitado}

A seguir definimos o conjunto que entendemos como domínio de uma densidade.

Definição 2.1.1. Definimos como domínio de uma densidade o conjunto de pontos para os quais a densidade não é nula.

Durante todo o trabalho, assumimos que a densidade bivariada é contínua no domínio. A densidade marginal da variavel $X$ é denotada por $f_{X}$ e a densidade de $Y$ por $g_{Y}$.

Neste caso de domínio limitado, a aproximação é conseqüência do Teorema de StoneWeierstrass; ver, por exemplo, Rudin (1976) ou Lages Lima (2003). Em particular, a apro- 
ximação é obtida a partir de um dos seus corolários que afirma que uma função contínua com suporte compacto é aproximada uniformemente por uma seqüência de polinômios.

Proposição 2.1.2. Se $h:[a, b] \times[a, b] \mapsto] 0,+\infty[$ é uma função contínua, então pode ser aproximada uniformemente por uma seqüência de exponencias de polinômios nas variáveis $x$ e $y$, isto é, para todo $\epsilon>0$ existe um polinômio $P_{n}(x, y)=\sum_{i, j=0}^{n} \lambda_{i j} x^{i} y^{j}$ tal que

$$
\left|h(x, y)-\exp \left(P_{n}(x, y)\right)\right|<\epsilon \quad \text { para todo } \quad(x, y) \in[a, b] \times[a, b]
$$

sendo $\lambda_{i j}$, para $i, j=0, \cdots, n$, números reais.

Prova: Ver a demonstração no Apêndice B.

No seguinte resultado vemos que a seqüência da proposição anterior pode ser modificada para uma seqüência de densidades no caso em que a própria função aproximada seja uma densidade, ou seja, que tenha integral total um.

Lema 2.1.3. Se h é uma densidade com domínio $[a, b] \times[a, b]$, contínua no domínio, então pode ser aproximada uniformemente por uma seqüência de densidades definidas por exponenciais de polinômios nas variáveis $x$ e $y$.

Prova: Ver a demonstração no Apêndice B.

Os resultados anteriores podem ser resumidos no seguinte corolário, no qual também analisamos a convergência das correspondentes densidades marginais.

Corolário 2.1.4. Seja $h$ uma densidade contínua no domínio $[a, b] \times[a, b]$ com marginais $f_{X}$ e $g_{Y}$. Para todo $\epsilon>0$, existe um polinômio $P_{n}(x, y)=\sum_{i, j=0}^{n} \lambda_{i j} x^{i} y^{j}$ tal que

$$
\begin{aligned}
& \max _{(x, y) \in[a, b] \times[a, b]}\left|h(x, y)-\exp \left(P_{n}(x, y)\right)\right|<\epsilon, \\
& \max _{x \in[a, b]}\left|f_{X}(x)-\int_{a}^{b} \exp \left(P_{n}(x, y)\right) d y\right|<\epsilon \quad e \\
& \max _{y \in[a, b]}\left|g_{Y}(y)-\int_{a}^{b} \exp \left(P_{n}(x, y)\right) d x\right|<\epsilon .
\end{aligned}
$$


Prova: Conseqüência do Lema 2.1.3. As desigualdades das marginais seguem do fato de $h$ ter domínio limitado e a aproximação por exponenciais de polinômios ser uniforme.

Assim como no corolário anterior, também podemos concluir que se $h \approx \exp \left(P_{n}\right)$, então para quaisquer $r, s \in \mathbb{N}$ (N denota o conjunto dos números naturais)

$$
\int_{a}^{b} \int_{a}^{b} x^{r} y^{s} \exp (P(x, y)) d x d y
$$

é próxima de

$$
\mathbb{E}\left[X^{r} Y^{s}\right]=\int_{a}^{b} \int_{a}^{b} x^{r} y^{s} h(x, y) d x d y
$$

Este fato segue do domínio de $h$ ser limitado e da aproximação por $\exp \left(P_{n}\right)$ ser uniforme.

É útil observar que quando $h$ tem domínio limitado também podemos definir a seguinte igualdade

$$
h(x, y)=M-\tilde{h}(x, y) \quad \text { para } \quad(x, y) \in[a, b] \times[a, b]
$$

sendo

$$
M=\max _{(x, y) \in[a, b] \times[a, b]} h(x, y)+\epsilon, \quad \epsilon>0 \quad \text { arbitrário e } \quad \tilde{h}(x, y)=M-h(x, y)
$$

Antes de prosseguir, observamos que a constante $M$ está bem definida pois a densidade conjunta $h$ é uma função contínua num conjunto limitado, então possui máximo. Essa constante implica que $\tilde{h}=M-h$ é uma função contínua e positiva para $(x, y) \in[a, b] \times[a, b]$ e portanto pode ser uniformemente aproximada pela exponencial de um polinômio nas variáveis $x$ e $y$ (Proposição 2.1.2). Consideramos então

$$
\tilde{h}(x, y) \approx \exp \left(\lambda_{0}+\sum_{\substack{i, j=0 \\ i+j \neq 0}}^{n} \lambda_{i j} x^{i} y^{j}\right)
$$

para algum $n \in \mathbb{N}$ sendo

$$
\lambda_{0}=\ln \left(\frac{M(b-a)^{2}-1}{\int_{a}^{b} \int_{a}^{b} \exp \left(\sum_{\substack{i, j=0 \\ i+j \neq 0}}^{n} \lambda_{i j} x^{i} y^{j}\right)}\right) .
$$


O coeficiente $\lambda_{0}$ está bem definido pois $M(b-a)^{2}-1>0$ já que

$$
1=\int_{a}^{b} \int_{a}^{b} h(x, y) d x d y \leq(b-a)^{2} \max _{(x, y) \in[a, b] \times[a, b]} h(x, y)<M(b-a)^{2} .
$$

Denotamos as densidades marginais de $\tilde{h}$ por $\tilde{f}_{X}$ e $\tilde{g}_{Y}$, isto é

$$
\tilde{f}_{X}(x)=\int_{a}^{b} \tilde{h}(x, y) d y \quad \text { e } \quad \tilde{g_{Y}}(y)=\int_{a}^{b} \tilde{h}(x, y) d x
$$

A vantagem de trabalhar com esta outra expressão de $h$, radica na determinação dos coeficientes $\lambda_{i j}, i, j=0, \ldots, n$ segundo a convexidade das marginais. Nas aproximações estudadas no capítulo 3, principalmente na seção §3.2.1, resulta mais simples obter os coeficientes que reproduzem uma marginal convexa do que uma côncava. Porém, na modelagem aplicada, são mais usadas as densidades unimodais, ou seja côncavas, do que as convexas. Essa aparente desvantagem da metodologia proposta pode ser superada utilizando a relação $h=M-\tilde{h}$. Logo, a partir do comportamento geométrico das marginais, determinamos os coeficientes de $\tilde{h}$, sendo que as marginais de $\tilde{h}$ são convexas se, e somente se, as correspondentes de $h$ são côncavas. Por exemplo, se a densidade $f_{X}$ (correspondente a $h$ ) for unimodal, determinamos os coeficientes de $\tilde{h}$ de tal forma que a densidade $\tilde{f}_{X}$ (correspondente a $\tilde{h}$ ) seja uma função com um ponto mínimo. Um exemplo numérico pode ser visto na Figura 3.2.6. Ao aplicar a relação $h=M-\tilde{h}$ pode acontecer que não tenhamos informação prévia do valor da constante $M$, ou seja, que não conheçamos o valor máximo da densidade conjunta que estamos aproximando. Nesse caso, pode ser utilizado qualquer valor para $M$ desde que $M>\frac{1}{(b-a)^{2}}$, por exemplo $M=\frac{2}{(b-a)^{2}}$.

Na seguinte seção, analisamos o caso em que o domínio de $h$ é ilimitado.

\subsection{Domínio ilimitado}

O objetivo desta seção é provar a aproximação uniforme de uma densidade bivariada contínua com domínio ilimitado por uma seqüência de exponenciais de polinômios. Algumas hipóteses sobre a densidade são necessárias, mas salientamos que são hipóteses simples que excluem casos raros. Denotamos por $\mathbb{R}$ o conjunto dos números reais. 
Definição 2.2.1. Seja $h: \mathbb{R} \times \mathbb{R} \mapsto] 0,+\infty[$ uma densidade. Dizemos que $h \in \mathcal{H}$ se $h$ é contínua, possui densidades marginais contínuas e as seguintes condições são satisfeitas

i) $\lim _{\|(x, y)\| \rightarrow+\infty} h(x, y)=0$,

ii) $\lim _{|x| \rightarrow+\infty} f_{X}(x)=0$,

iii) $\lim _{|y| \rightarrow+\infty} g_{Y}(y)=0$,

onde $\|(x, y)\|$ denota a norma euclideana de $(x, y)$, isto é, $\|(x, y)\|=\sqrt{x^{2}+y^{2}}$.

Um exemplo de densidade conjunta em $\mathcal{H}$ é a densidade normal bivariada. Por outro lado, podemos ver que as condições i)-iii) não são redundantes já que em Romano and Siegel (1986) $\S 2.7$ é dado o seguinte exemplo de uma densidade contínua que não tende assintoticamente a zero. Seja $Z$ uma variável com distribuição geométrica, ou seja $\mathbb{P}(Z=n)=2^{-n}$, para $n=1,2, \ldots$, e seja $W$ uma outra variável com distribuição condicionada a $Z$, Gaussiana com média $Z$ e variância $n^{-2} 2^{-2 n}$. Então a distribuição, não condicionada, de $W$ é uma mistura de tais distribuições Gaussianas e a densidade é a soma delas, isto é,

$$
f_{W}(w)=\sum_{i=1}^{\infty} \frac{n 2^{n}}{\sqrt{2 \pi}} \exp \left(-\frac{n 2^{n}(w-n)^{2}}{2}\right) .
$$

A densidade possui domínio ilimitado, é contínua mas não tende para zero quando $w$ tende para infinito. Na Figura 2.2.1 mostramos uma aproximação da densidade $f_{W}$.

Uma outra observação interessante, acerca da definição do conjunto de densidades $\mathcal{H}$, é que a condição das densidades conjuntas contínuas terem marginais contínuas também não é redundante. Novamente, em Romano and Siegel (1986) §2.15, é apresentado um exemplo de densidade bivariada com domínio ilimitado e contínua que possui uma das marginais descontínua. A densidade

$$
h(w, z)= \begin{cases}\frac{1}{\sqrt{2 \pi}} \exp \left(-w-\frac{\left(z-\left(\frac{1}{w}\right)^{2}\right)}{2}\right), & \text { se } w>0 \\ 0, & \text { se } w \leq 0\end{cases}
$$

é contínua em $\mathbb{R} \times \mathbb{R}$, porém a densidade marginal $f_{W}(w)=\exp (-w) \mathbf{1}_{\{w>0\}}$ é descontínua na origem. 
Figura 2.2.1

Densidade $f_{W}(w)=\sum_{i=1}^{\infty} \frac{n 2^{n}}{\sqrt{2 \pi}} \exp \left(-\frac{n 2^{n}(w-n)^{2}}{2}\right)$.

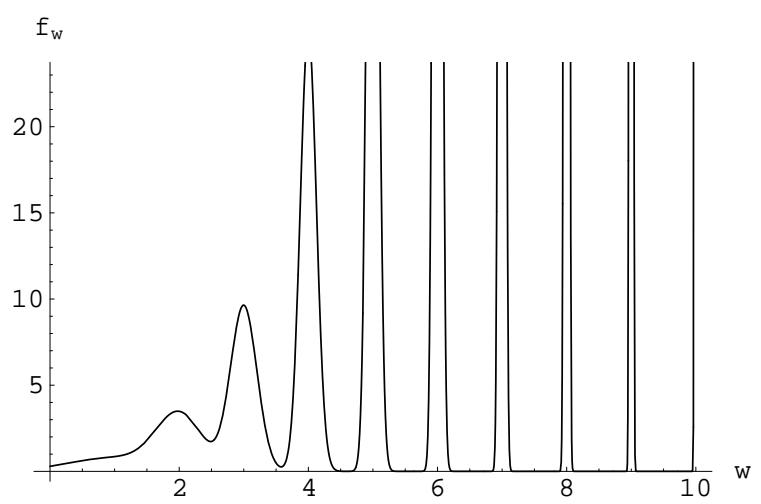

Uma vez estabelecidas as características das densidades que podem ser aproximadas, começamos a dar os resultados necessários para provar a aproximação. A seguir, fornecemos um roteiro da apresentação de tais resultados. Primeiramente, no Lema 2.2.2 e na Proposição 2.2.3 descrevemos algumas das propriedades das densidades do conjunto $\mathcal{H}$. Dada uma densidade conjunta $h \in \mathcal{H}$, estudamos a convergência da seqüência definida por truncamentos de $h$, especificamente, $\lim _{n \rightarrow+\infty} h_{n}=h$. Como segundo passo, aplicamos os resultados da seção anterior e concluímos que cada densidade $h_{n}$, obtida por um truncamento de $h$, pode ser aproximada por uma seqüência de exponenciais de polinômios $\left(\exp \left(P_{m}^{n}\right)\right)_{m \in \mathbb{N}}$, sendo $\lim _{m \rightarrow+\infty} \exp \left(P_{m}^{n}\right)=h_{n}$. O terceiro passo é o Teorema 2.2.6, no qual mostramos que a seqüência definida pela diagonal, ou seja $\left(\exp \left(P_{n}^{n}\right)\right)_{n \in \mathbb{N}}$, aproxima $h$. Finalmente, no Corolário 2.2.8 e na Proposição 2.2.9, provamos que as probabilidades e momentos calculados com a seqüência aproximante convergem aos calculados com $h$.

Definimos a seqüência de funções $\left(h_{n}\right)_{n \in \mathbb{N}}$ como segue

$$
\begin{aligned}
h_{n} & : \mathbb{R} \times \mathbb{R} \mapsto \mathbb{R} \\
h_{n}(x, y) & =h(x, y) \mathbf{1}_{[-n, n]}(x) \mathbf{1}_{[-n, n]}(y)
\end{aligned}
$$

denotando por 1 a função indicadora usual. A partir de $\left(h_{n}\right)_{n \in \mathbb{N}}$ obtemos as correspondentes 
seqüências de funções marginais $\left(f_{X}^{n}\right)_{n \in \mathbb{N}}$ and $\left(g_{Y}^{n}\right)_{n \in \mathbb{N}}$ sendo

$$
\begin{aligned}
& f_{X}^{n}(x)=\int_{-\infty}^{+\infty} h_{n}(x, y) d y=\mathbf{1}_{[-n, n]}(x) \int_{-n}^{n} h(x, y) d y \\
& g_{Y}^{n}(y)=\int_{-\infty}^{+\infty} h_{n}(x, y) d x=\mathbf{1}_{[-n, n]}(y) \int_{-n}^{n} h(x, y) d x .
\end{aligned}
$$

Denominamos por seqüência truncada de $h$ a seqüência $\left(h_{n}\right)_{n \in \mathbb{N}}$ junto com as correspondentes seqüências $\left(f_{X}^{n}\right)_{n \in \mathbb{N}}$ e $\left(g_{Y}^{n}\right)_{n \in \mathbb{N}}$.

O nosso primeiro resultado é uma propriedade das densidades em $\mathcal{H}$.

Lema 2.2.2. Seja $h: \mathbb{R} \times \mathbb{R} \mapsto] 0,+\infty[$ uma densidade em $\mathcal{H}$. Então valem as seguintes convergências

1. $\lim _{n \rightarrow+\infty}\left(\int_{-\infty}^{-n} h(x, y) d y+\int_{n}^{+\infty} h(x, y) d y\right)=0$, uniformemente em $x$ para $x \in \mathbb{R}$;

2. $\lim _{n \rightarrow+\infty}\left(\int_{-\infty}^{-n} h(x, y) d x+\int_{n}^{+\infty} h(x, y) d x\right)=0$, uniformemente em $y$ para $y \in \mathbb{R}$.

Prova: Ver a demonstração no Apêndice B.

A seguinte proposição é a principal propriedade das densidades em $\mathcal{H}$.

Proposição 2.2.3. Seja $h: \mathbb{R} \times \mathbb{R} \mapsto] 0,+\infty[$ uma densidade em $\mathcal{H}$. Para a seqüência truncada de $h$ e qualquer $\epsilon>0$, existe um $n_{0} \in \mathbb{N}$ tal que para $n \geq n_{0}$

$$
\begin{aligned}
\sup _{(x, y) \in \mathbb{R} \times \mathbb{R}}\left|h(x, y)-h_{n}(x, y)\right| & <\epsilon, \\
& \sup _{x \in \mathbb{R}}\left|f_{X}(x)-f_{X}^{n}(x)\right|<\epsilon e \\
& \sup _{y \in \mathbb{R}}\left|g_{Y}(y)-g_{Y}^{n}(y)\right|<\epsilon .
\end{aligned}
$$

Prova: Ver a demonstração no Apêndice B.

Agora aplicamos em cada elemento da seqüência truncada de $h$ os resultados da seção anterior para densidades de domínio limitado. 
Lema 2.2.4. Seja $\left(h_{n}\right)_{n \in \mathbb{N}}$ a seqüência truncada de $h$, com $h \in \mathcal{H}$. Para cada $n \in \mathbb{N}$, existe uma seqüência $\left(\mathcal{E}_{m}^{n}\right)_{m \in \mathbb{N}}$ tal que

$$
\begin{aligned}
\sup _{(x, y) \in[-n, n] \times[-n, n]}\left|h_{n}(x, y)-\mathcal{E}_{m}^{n}(x, y)\right| & <\frac{1}{m^{3}}, \\
\sup _{x \in[-n, n]}\left|f_{X}^{n}(x)-f_{X}^{\mathcal{E}_{m}^{n}}(x)\right| & <\frac{1}{m^{3}} e \\
\sup _{y \in[-n, n]}\left|g_{Y}^{n}(y)-g_{Y}^{\mathcal{E}_{m}^{n}}(y)\right| & <\frac{1}{m^{3}},
\end{aligned}
$$

sendo $\mathcal{E}_{m}^{n}$ uma densidade dada pela exponencial de um polinômio em $x$ e $y$.

Prova: Conseqüência da Proposição 2.1.2.

Lema 2.2.5 (Seqüência diagonal). A seqüência $\left(\overline{\mathcal{E}}_{n}\right)_{n \in \mathbb{N}}$ definida por $\overline{\mathcal{E}}_{n} \equiv \mathcal{E}_{n}^{n}$ satisfaz as seguintes desigualdades

$$
\begin{aligned}
\sup _{(x, y) \in[-n, n] \times[-n, n]}\left|h_{n}(x, y)-\overline{\mathcal{E}}_{n}(x, y)\right| & <\frac{1}{n^{3}}, \\
\sup _{x \in[-n, n]}\left|f_{X}^{n}(x)-f_{X}^{\overline{\mathcal{E}}_{n}}(x)\right| & <\frac{1}{n^{3}} e \\
\sup _{y \in[-n, n]}\left|g_{Y}^{n}(y)-g_{Y}^{\overline{\mathcal{E}}_{n}}(y)\right| & <\frac{1}{n^{3}} .
\end{aligned}
$$

Prova: Segue da definição de $\overline{\mathcal{E}}_{n}$ e do Lema 2.2.4.

A seqüência diagonal $\left(\overline{\mathcal{E}}_{n}\right)_{n \in \mathbb{N}}$ definida no Lema 2.2 .5 é construída com o limitante $\frac{1}{n^{3}}$ para garantir a convergência das probabilidades (ver Corolário 2.2.8). Agora podemos enunciar o principal resultado da presente seção, o qual estabelece que a seqüência $\left(\overline{\mathcal{E}}_{n}\right)_{n \in \mathbb{N}}$ converge para $h \in \mathcal{H}$. 
Teorema 2.2.6. Seja $h: \mathbb{R} \times \mathbb{R} \mapsto] 0,+\infty\left[\right.$ uma densidade em $\mathcal{H}$, então a seqüência $\left(\overline{\mathcal{E}}_{n}\right)_{n \in \mathbb{N}}$ satisfaz que para todo $\epsilon>0$, existe um $n_{0} \in \mathbb{N}$ tal que se $n \geq n_{0}$, então

$$
\begin{aligned}
\sup _{(x, y) \in \mathbb{R} \times \mathbb{R}}\left|h(x, y)-\overline{\mathcal{E}}_{n}(x, y)\right| & <\frac{1}{n}, \\
\sup _{x \in \mathbb{R}}\left|f_{X}(x)-f_{X}^{\overline{\mathcal{E}}_{n}}(x)\right| & <\frac{1}{n} e \\
\sup _{y \in \mathbb{R}}\left|g_{Y}(y)-g_{Y}^{\overline{\mathcal{E}}_{n}}(y)\right| & <\frac{1}{n} .
\end{aligned}
$$

Prova: Ver a demonstração no Apêndice B.

O Lema 2.2.5 e o Teorema 2.2.6 continuam valendo se consideramos $\left(h_{n}\right)_{n \in \mathbb{N}}$ e $\left(\overline{\mathcal{E}}_{n}\right)_{n \in \mathbb{N}}$ funções de densidades, isto é, funções com integral total 1.

Finalmente verificamos que a seqüência aproximante construída está bem definida no sentido de que as probabilidades calculadas com $\left(\overline{\mathcal{E}}_{n}\right)_{n \in \mathbb{N}}$ convergem para a probabilidade correspondente de $h$. Mais especificamente, provamos que para quaisquer $a_{x}, b_{x}, a_{y}, b_{y}$ tais que $-\infty<a_{x}<b_{x}<+\infty$ e $-\infty<a_{y}<b_{y}<+\infty$, a seqüência

$$
\mathbb{P}_{\overline{\mathcal{E}}_{n}}\left(a_{x}<X<b_{x}, a_{y}<Y<b_{y}\right)=\int_{a_{x}}^{b_{x}} \int_{a_{y}}^{b_{y}} \overline{\mathcal{E}}_{n}(x, y) d y d x
$$

converge para

$$
\mathbb{P}\left(a_{x}<X<b_{x}, a_{y}<Y<b_{y}\right)=\int_{a_{x}}^{b_{x}} \int_{a_{y}}^{b_{y}} h(x, y) d y d x .
$$

Observemos que

- Se $a_{x}, b_{x}, a_{y}, b_{y} \in \mathbb{R}$, então a convergência de (2.7) para (2.8) é conseqüência da convergência uniforme de $\overline{\mathcal{E}}_{n}$ para $h$;

- Se $a_{x}=-\infty, b_{x}=+\infty$ e $a_{y}, b_{y} \in \mathbb{R}$, então a convergência de (2.7) para (2.8) é consequiência da convergência uniforme de $g_{Y}^{\overline{\mathcal{E}}_{n}}$ para $g_{Y}$;

- Se $a_{y}=-\infty, b_{y}=+\infty$ e $a_{x}, b_{x} \in \mathbb{R}$, então a convergência de (2.7) para (2.8) é conseqüência da convergência uniforme de $f_{X}^{\overline{\mathcal{E}}_{n}}$ para $f_{X}$.

$\mathrm{Na}$ seguinte proposição enunciamos a convergência das probabilidades de alguns dos casos restantes. 
Proposição 2.2.7. A convergência

$$
\mathbb{P}_{\overline{\mathcal{E}}_{n}}\left(a_{x}<X<b_{x}, a_{y}<Y<b_{y}\right) \stackrel{n \rightarrow+\infty}{\longrightarrow} \mathbb{P}\left(a_{x}<X<b_{x}, a_{y}<Y<b_{y}\right)
$$

é satisfeita para os seguintes casos

1. $a_{x}=-\infty$ e $b_{x}, a_{y}, b_{y} \in \mathbb{R}$;

2. $a_{x}=a_{y}=-\infty$ e $b_{x}, b_{y} \in \mathbb{R}$;

3. $a_{x}=a_{y}=-\infty, b_{y}=+\infty$ e $b_{x} \in \mathbb{R}$.

Prova: Ver a demonstração no Apêndice B.

Pela proposição anterior, concluímos que a convergência das probabilidades é válida para todos os possíveis valores de $a_{x}, b_{x}, a_{y}$ e $b_{y}$.

Corolário 2.2.8. A convergência

$$
\mathbb{P}_{\overline{\mathcal{E}}_{n}}\left(a_{x}<X<b_{x}, a_{y}<Y<b_{y}\right) \stackrel{n \rightarrow+\infty}{\longrightarrow} \mathbb{P}\left(a_{x}<X<b_{x}, a_{y}<Y<b_{y}\right)
$$

é válida para quaisquer $a_{x}, b_{x}, a_{y}, b_{y}$ tais que $-\infty \leq a_{x}<b_{x} \leq+\infty e-\infty \leq a_{y}<b_{y} \leq+\infty$.

Prova: Conseqüência da Proposição 2.2.7 e das observações anteriores.

O último resultado do presente capítulo é sobre a convergência dos momentos. Observamos que no resultado da Proposição 2.2 .9 a seguir, a seqüência aproximante $\left(\overline{\mathcal{E}}_{n}\right)_{n \in \mathbb{N}}$ é construída com novos limitantes. Tais limitantes dependem do valor máximo da ordem dos momentos calculados, ou seja, depende do valor máximo de $r_{i}$ e $s_{i}$ para $i=1,2, \cdots, M$ (conforme à notação usada em dita proposição).

Proposição 2.2.9. Seja $h: \mathbb{R} \times \mathbb{R} \mapsto] 0,+\infty\left[\right.$ uma densidade em $\mathcal{H}$. Se $\mathbb{E}\left[X^{r_{i}} Y^{s_{i}}\right]<\infty$ para $s_{i}, r_{i} \in \mathbb{N} \cup\{0\}$ e $i=1,2, \cdots, M$ sendo $M \in \mathbb{N}$, então existe uma seqüência diagonal $\left(\overline{\mathcal{E}}_{n}\right)_{n \in \mathbb{N}}$ que converge uniformemente para $h$, assim como as correspondentes marginais e probabilidades, $e$

converge para

$$
\mathbb{E}_{\overline{\mathcal{E}}_{n}}\left[X^{r_{i}} Y^{s_{i}}\right]=\int_{-n}^{n} \int_{-n}^{n} x^{r_{i}} y^{s_{i}} \overline{\mathcal{E}}_{n}(x, y) d y d x
$$

$$
\mathbb{E}\left[X^{r_{i}} Y^{s_{i}}\right]=\int_{-\infty}^{+\infty} \int_{-\infty}^{+\infty} x^{r_{i}} y^{s_{i}} h(x, y) d y d x
$$

para todo $i=1,2, \cdots, M$. 
Prova: Ver a demonstração no Apêndice B.

Concluímos a análise da aproximação para domínio ilimitado afirmando que os resultados do presente capítulo também são válidos quando o domínio da densidade conjunta é da forma $[a,+\infty[\times[a,+\infty[$ ou da forma $]-\infty, a] \times]-\infty, a]$, sendo $a$ um número real qualquer.

As propriedades enunciadas e demonstradas neste capítulo são originais, salvo aquelas nas quais damos a referência da bibliografía consultada. Salientamos que as técnicas de aproximação expostas são muito utilizadas em outras áreas de pesquisa, por exemplo, em análise. A maioria dos resultados do presente capítulo foi apresentada em um dos seminários de Séries Temporais, Análise de Depêndencia e Aplicações em Atuária e Finanças realizados no Instituto de Matemática e Estatística da Universidade de São Paulo.

Nos seguintes capítulos assumimos que a densidade conjunta possui domínio limitado. Com o exposto na seção 2.2 vemos que tal suposição não é restritiva, pois em caso da densidade ser de domínio ilimitado, aplicamos os resultados para a densidade aproximante que possui domínio limitado. 


\section{Capítulo 3}

\section{Comportamento geométrico das densidades marginais}

O objetivo deste capítulo é mostrar os conjuntos de possíveis valores dos coeficientes da aproximação de uma densidade conjunta segundo a natureza geométrica das marginais.

Especificamente, consideramos os casos quando a densidade bivarida $h(x, y)$ pode ser descrita por

$$
h(x, y)=\exp \left(\lambda_{0}+\lambda_{10} x+\lambda_{01} y+\lambda_{11} x y+\lambda_{20} x^{2}+\lambda_{02} y^{2}\right), \quad(x, y) \in[a, b] \times[a, b]
$$

ou por

$$
h(x, y)=\exp \left(\lambda_{0}+\lambda_{10} x+\lambda_{01} y+\lambda_{11} x y+\lambda_{21} x^{2} y\right), \quad(x, y) \in[a, b] \times[a, b]
$$

sendo $[a, b] \subset \mathbb{R}$. Pela informação dada sobre o comportamento geométrico das marginais, determinamos os intervalos onde os coeficientes $\lambda_{10}, \lambda_{01}, \lambda_{11}, \lambda_{20}, \lambda_{02}$ e $\lambda_{21}$ podem ser definidos ( $\lambda_{0}$ é simplesmente uma constante normalizadora). Dividimos a análise do problema em questão segundo a nulidade dos coeficientes $\lambda_{11}, \lambda_{20}, \lambda_{02}$ e $\lambda_{21}$.

Usamos a seguinte notação para as densidades marginais

$$
f_{X}(x)=\int_{a}^{b} h(x, y) d y, \quad g_{Y}(y)=\int_{a}^{b} h(x, y) d x
$$

e denotamos 
- $f_{X}^{c}$ e $g_{Y}^{c}$, as densidades constantes;

- $f_{X}^{i}$ e $g_{Y}^{i}$, as densidades crescentes;

- $f_{X}^{d}$ e $g_{Y}^{d}$, as densidades decrescentes;

- $f_{X}^{m}$ e $g_{Y}^{m}$, as densidades com um ponto mínimo.

Observemos que por ser a densidade conjunta uma função exponencial num domínio limitado, as correspondentes densidades marginais $f_{X}$ e $g_{Y}$ são funções contínuas e positivas nos respectivos domínios. Quando falarmos de serem crescentes, decrescentes, etc., tais propriedades serão consideradas nos correspondentes domínios.

\subsection{Polinômio de grau um}

Começamos a nossa abordagem do problema com o caso simples da densidade dada pela equação (3.1) quando $\lambda_{11}=\lambda_{20}=0=\lambda_{02}$, ou seja $h(x, y)=\exp \left(\lambda_{0}+\lambda_{10} x+\lambda_{01} y\right)$ para $(x, y) \in[a, b] \times[a, b]$. É fácil obter os intervalos nos quais devem estar os coeficientes $\lambda_{10}$ e $\lambda_{01}$ segundo a possível natureza das marginais. A Tabela 3.1.1 apresenta os resultados obtidos.

Por exemplo, podemos ver na Tabela 3.1.1 que para o caso de densidades crescentes, isto é $f_{X}^{i}$ e $g_{Y}^{i}$, os únicos valores possíveis para os coeficientes $\lambda_{10}$ e $\lambda_{01}$ são os números reais positivos.

\section{Tabela 3.1.1}

Condições encontradas para os coeficientes $\lambda_{10}$ e $\lambda_{01}$ dadas as diferentes densidades marginais de $h(x, y)=\exp \left(\lambda_{0}+\lambda_{10} x+\lambda_{01} y\right)$.

\begin{tabular}{|c|c|c|c|}
\hline & $g_{Y}^{c}$ & $g_{Y}^{i}$ & $g_{Y}^{d}$ \\
\hline \hline$f_{X}^{c}$ & $\lambda_{10}=0$ e $\lambda_{01}=0$ & $\lambda_{10}=0$ e $\lambda_{01}>0$ & $\lambda_{10}=0$ e $\lambda_{01}<0$ \\
\hline$f_{X}^{i}$ & $\lambda_{10}>0$ e $\lambda_{01}=0$ & $\boldsymbol{\lambda}_{\mathbf{1 0}}>\mathbf{0}$ e $\boldsymbol{\lambda}_{\mathbf{0 1}}>\mathbf{0}$ & $\lambda_{10}>0$ e $\lambda_{01}<0$ \\
\hline$f_{X}^{d}$ & $\lambda_{10}<0$ e $\lambda_{01}=0$ & $\lambda_{10}<0$ e $\lambda_{01}>0$ & $\lambda_{10}<0$ e $\lambda_{01}<0$ \\
\hline
\end{tabular}




\subsection{Polinômio de grau dois}

\subsubsection{Família de três parâmetros}

Analisamos a seguir a representação (3.1) quando $\lambda_{11} \neq 0$ e $\lambda_{20}=0=\lambda_{02}$. Neste caso a densidade conjunta é dada por $h(x, y)=\exp \left(\lambda_{0}+\lambda_{10} x+\lambda_{01} y+\lambda_{11} x y\right)$, para $(x, y) \in[a, b] \times[a, b]$ e as marginais correspondentes são

$$
f_{X}(x)= \begin{cases}\exp \left(\lambda_{0}+\lambda_{10} x\right)\left(\frac{\exp \left(\left(\lambda_{01}+\lambda_{11} x\right) b\right)-\exp \left(\left(\lambda_{01}+\lambda_{11} x\right) a\right)}{\lambda_{01}+\lambda_{11} x}\right), & \text { se } \lambda_{01}+\lambda_{11} x \neq 0 \\ \exp \left(\lambda_{0}+\lambda_{10} x\right)(b-a), & \text { se } \lambda_{01}+\lambda_{11} x=0\end{cases}
$$

e

$$
g_{Y}(y)= \begin{cases}\exp \left(\lambda_{0}+\lambda_{01} y\right)\left(\frac{\exp \left(\left(\lambda_{10}+\lambda_{11} y\right) b\right)-\exp \left(\left(\lambda_{10}+\lambda_{11} y\right) a\right)}{\lambda_{10}+\lambda_{11} y}\right), & \text { se } \lambda_{10}+\lambda_{11} y \neq 0 \\ \exp \left(\lambda_{0}+\lambda_{01} y\right)(b-a), & \text { se } \lambda_{10}+\lambda_{11} y=0 .\end{cases}
$$

Estamos interessados no sinal da derivada de $f_{X}$ e $g_{Y}$, pois a derivada positiva está associada à função crescente, a derivada negativa à função decrescente e a derivada nula à função constante ou a possibilidade de função com ponto máximo ou mínimo. As derivadas podem ser estudadas usando a aplicação seguinte

$$
\psi(z) \stackrel{\text { def }}{=} \begin{cases}\frac{(b-a) \exp ((b-a) z)}{\exp ((b-a) z)-1}-\frac{1}{z}, & \text { se } z \neq 0 \\ \frac{b-a}{2}, & \text { se } z=0\end{cases}
$$

pois temos as relações

$$
\frac{d f_{X}}{d x}(x)=f_{X}(x)\left(\lambda_{11} \psi\left(\lambda_{01}+\lambda_{11} x\right)+\lambda_{10}+a \lambda_{11}\right)
$$

e

$$
\frac{d g_{Y}}{d y}(y)=g_{Y}(y)\left(\lambda_{11} \psi\left(\lambda_{10}+\lambda_{11} y\right)+\lambda_{01}+a \lambda_{11}\right)
$$


Tais relações são provadas na Proposição C.1 do Apêndice C. Com isso obtemos por exemplo

$$
\frac{d f_{X}}{d x}(x)>0 \Leftrightarrow-\lambda_{11} \psi\left(\lambda_{01}+\lambda_{11} x\right)-a \lambda_{11}<\lambda_{10} .
$$

A seguinte proposição enuncia as principais propriedades da função $\psi$.

Proposição 3.2.1. A função $\psi$ é contínua, estritamente crescente e limitada como segue

$$
0<\psi(z)<b-a \quad \text { para } \quad z \in \mathbb{R} .
$$

Prova: A demonstração pode ser vista nos Corolários A.2 e A.3 do Apêndice A, substituindo a constante $c$ dos ditos corolários por $b-a$.

Observemos que a expressão $\psi\left(\lambda_{01}+\lambda_{11} x\right)$ é uma função crescente em $x$ para $\lambda_{11}>0 \mathrm{e}$ decrescente para $\lambda_{11}<0$. Analogamente, $\psi\left(\lambda_{10}+\lambda_{11} y\right)$ é uma função crescente em $y$ para $\lambda_{11}>0$ e decrescente para $\lambda_{11}<0$. Isto nos permite concluir as seguintes relações válidas para todo $x, y \in] a, b\left[\right.$ e $\lambda_{10}, \lambda_{01}, \lambda_{11} \in \mathbb{R} \operatorname{com} \lambda_{11} \neq 0$,

$$
\lambda_{11} \psi\left(\lambda_{01}+\lambda_{11} a\right)<\lambda_{11} \psi\left(\lambda_{01}+\lambda_{11} x\right)<\lambda_{11} \psi\left(\lambda_{01}+\lambda_{11} b\right)
$$

$\mathrm{e}$

$$
\lambda_{11} \psi\left(\lambda_{10}+\lambda_{11} a\right)<\lambda_{11} \psi\left(\lambda_{10}+\lambda_{11} y\right)<\lambda_{11} \psi\left(\lambda_{10}+\lambda_{11} b\right) .
$$

Com o intuito de simplificar a notação na hora de estabelecer as relações entre os coeficientes $\lambda_{10}, \lambda_{01}$ e $\lambda_{11}$ definimos, para cada $\lambda_{11}$ não nulo, a seguinte função

$$
L\left(z, \lambda ; \lambda_{11}\right) \stackrel{\text { def }}{=}-\lambda_{11}\left(\psi\left(\lambda+z \lambda_{11}\right)+a\right) \quad \text { para } z, \lambda \in \mathbb{R} .
$$

A partir das propriedades de $\psi$ obtemos as propriedades de $L\left(z, \lambda ; \lambda_{11}\right)$ enunciadas na seguinte proposição.

Proposição 3.2.2. A função $L\left(z, \lambda ; \lambda_{11}\right)$ é contínua. É decrescente na variável $z$ e, na variável $\lambda$, é decrescente para $\lambda_{11}>0$ e crescente para $\lambda_{11}<0$. Também é limitada como segue

$$
\begin{cases}-b \lambda_{11}<L\left(z, \lambda ; \lambda_{11}\right)<-a \lambda_{11}, & \text { se } \lambda_{11}>0 \\ -a \lambda_{11}<L\left(z, \lambda ; \lambda_{11}\right)<-b \lambda_{11}, & \text { se } \lambda_{11}<0 .\end{cases}
$$


Prova: Ver a demonstração no Apêndice C.

Escrevendo as relações (3.4) e (3.5) para a função $L$ obtemos

$$
L\left(a, \lambda_{01} ; \lambda_{11}\right)>L\left(x, \lambda_{01} ; \lambda_{11}\right)>L\left(b, \lambda_{01} ; \lambda_{11}\right)
$$

$\mathrm{e}$

$$
L\left(a, \lambda_{10} ; \lambda_{11}\right)>L\left(x, \lambda_{10} ; \lambda_{11}\right)>L\left(b, \lambda_{10} ; \lambda_{11}\right) .
$$

Definimos $L\left(z, \lambda ; \lambda_{11}\right)$ pois nos permite escrever de forma sucinta as relações entre os coeficientes segundo os diferentes comportamentos geométricos das marginais. Os casos de marginais crescentes e decrescentes estão resumidos na Tabela 3.2.1. Como exemplo do procedimento para obter as relações existentes, apresentamos o caso da marginal $f_{X}$ ser crescente. Para isto precisamos enunciar a seguinte propriedade da segunda derivada das marginais, na qual vemos que as marginais da densidade conjunta que estamos considerando, $h(x, y)=\exp \left(\lambda_{0}+\lambda_{10} x+\lambda_{01} y+\lambda_{11} x y\right)$, são funções estritamente convexas. A convexidade é equivalente à segunda derivada ser não negativa. Se a segunda derivada for positiva então obtemos convexidade estrita, fato detalhado, por exemplo, em Lages Lima (2002).

Proposição 3.2.3. As densidades marginais $f_{X}$ e $g_{Y}$ são funções estritamente convexas.

Prova: Ver a demonstração no Apêndice C.

Por serem funções com segunda derivada positiva em todo ponto do intervalo ] $a, b[$, podemos afirmar que as densidades marginais são crescentes se, e somente se, a derivada primeira é positiva. Obtendo assim a relação seguinte

$$
\begin{array}{rlr}
f_{X}^{i} & \Leftrightarrow \frac{d f_{X}}{d x}(x)>0 & \text { para todo } x \in] a, b[ \\
& \Leftrightarrow f_{X}(x)\left(\lambda_{11} \psi\left(\lambda_{01}+\lambda_{11} x\right)+\lambda_{10}+a \lambda_{11}\right)>0 \\
& \Leftrightarrow\left(\lambda_{11} \psi\left(\lambda_{01}+\lambda_{11} x\right)+\lambda_{10}+a \lambda_{11}\right)>0 \\
& \Leftrightarrow \lambda_{10}>-\lambda_{11} \psi\left(\lambda_{01}+\lambda_{11} x\right)-a \lambda_{11} \\
& \left.\Leftrightarrow \lambda_{10}>L\left(x, \lambda_{01} ; \lambda_{11}\right) \quad \text { para todo } x \in\right] a, b[.
\end{array}
$$


Como a função $L$ é contínua, decrescente em $x$ e satisfaz $L\left(a, \lambda_{01} ; \lambda_{11}\right)>L\left(x, \lambda_{01} ; \lambda_{11}\right)$ para qualquer $x \in] a, b\left[\right.$ podemos concluir que $f_{X}^{i} \Leftrightarrow L\left(a, \lambda_{01} ; \lambda_{11}\right) \leq \lambda_{10}$.

\section{Tabela 3.2 .1}

Condições encontradas para os coeficientes $\lambda_{10}, \quad \lambda_{01}$ e $\lambda_{11}$ dadas marginais crescentes ou decrescentes de $h(x, y)=\exp \left(\lambda_{0}+\lambda_{10} x+\lambda_{01} y+\lambda_{11} x y\right)$.

\begin{tabular}{|c|c|c|}
\hline & $g_{Y}^{i}$ & $g_{Y}^{d}$ \\
\hline \hline & $L\left(a, \lambda_{01} ; \lambda_{11}\right) \leq \lambda_{10}$ & $L\left(a, \lambda_{01} ; \lambda_{11}\right) \leq \lambda_{10}$ \\
$f_{X}^{i}$ & $\mathrm{e}$ & $\mathrm{e}$ \\
& $L\left(a, \lambda_{10} ; \lambda_{11}\right) \leq \lambda_{01}$ & $L\left(b, \lambda_{10} ; \lambda_{11}\right) \geq \lambda_{01}$ \\
\hline & $L\left(b, \lambda_{01} ; \lambda_{11}\right) \geq \lambda_{10}$ & $L\left(b, \lambda_{01} ; \lambda_{11}\right) \geq \lambda_{10}$ \\
$f_{X}^{d}$ & $\mathrm{e}$ & $\mathrm{e}$ \\
& $L\left(a, \lambda_{10} ; \lambda_{11}\right) \leq \lambda_{01}$ & $L\left(b, \lambda_{10} ; \lambda_{11}\right) \geq \lambda_{01}$ \\
\hline
\end{tabular}

As relações apresentadas na Tabela 3.2.1, junto com as desigualdades da Proposição 3.2.2, permitem inferir condições mais simples sobre os coeficientes para os diferentes comportamentos das marginais. Tais condições são dadas na seguinte proposição.

Proposição 3.2.4. Sejam $\lambda_{10}, \lambda_{01}$ e $\lambda_{11}$ números reais.

1. Para $\lambda_{11}>0$ temos que

(a) $-a \lambda_{11} \leq \lambda_{10}$ implica que a marginal $f_{X}$ é uma função crescente;

(b) $-b \lambda_{11} \geq \lambda_{10}$ implica que a marginal $f_{X}$ é uma função decrescente;

(c) $-a \lambda_{11} \leq \lambda_{01}$ implica que a marginal $g_{Y}$ é uma função crescente;

(d) $-b \lambda_{11} \geq \lambda_{01}$ implica que a marginal $g_{Y}$ é uma função decrescente. 
2. Para $\lambda_{11}<0$ temos que

(a) $-b \lambda_{11} \leq \lambda_{10}$ implica que a marginal $f_{X}$ é uma função crescente;

(b) $-a \lambda_{11} \geq \lambda_{10}$ implica que a marginal $f_{X}$ é uma função decrescente;

(c) $-b \lambda_{11} \leq \lambda_{01}$ implica que a marginal $g_{Y}$ é uma função crescente;

(d) $-a \lambda_{11} \geq \lambda_{01}$ implica que a marginal $g_{Y}$ é uma função decrescente.

Prova: Ver a demonstração no Apêndice C.

Na Figura 3.2.2 apresentamos uma possível representação de uma densidade conjunta com ambas marginais crescentes.

Figura 3.2.2

Densidade $h(x, y)=\exp (-2,927+2 x+2 y+x y)$ para $(x, y) \in[-1,1] \times[-1,1]$.

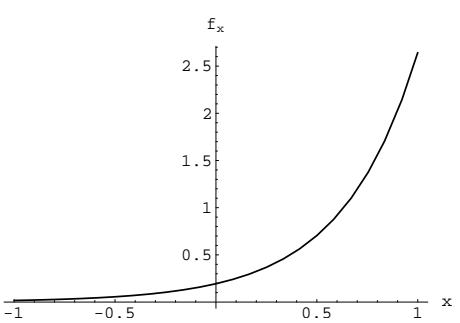

(a) Densidade marginal $f_{X}$.

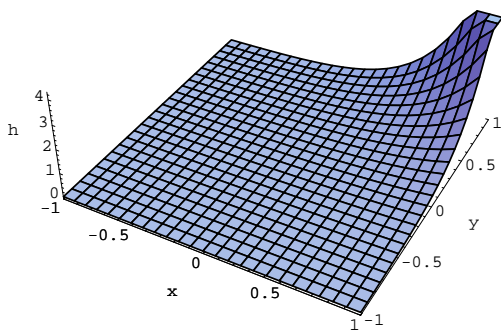

(c) Densidade conjunta $h$.

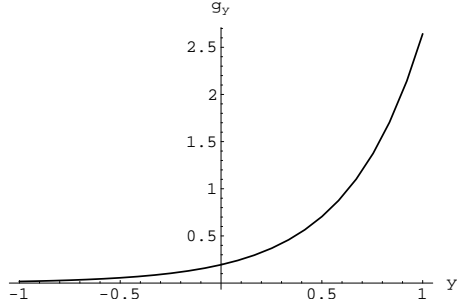

(b) Densidade marginal $g_{Y}$.

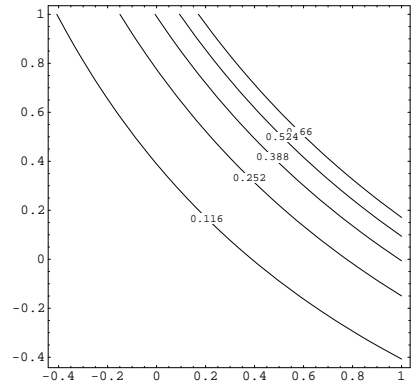

(d) Curvas de nível da densidade conjunta. 
Sendo ambas marginais convexas, é natural se perguntar quais as condições nos coeficientes para representar marginais com pontos mínimos. A resposta é dada na proposição a seguir.

Proposição 3.2.5. Sejam $\left.x_{0}, y_{0} \in\right] a, b[$.

1. $f_{X}$ possui um mínimo em $x_{0}$ se, e somente se, $L\left(x_{0}, \lambda_{01} ; \lambda_{11}\right)=\lambda_{10}$.

2. $g_{Y}$ possui um minimo em $y_{0}$ se, e somente se, $L\left(y_{0}, \lambda_{10} ; \lambda_{11}\right)=\lambda_{01}$.

Prova: Ver a demonstração no Apêndice C.

A partir do conjunto das propriedades enunciadas anteriormente, encontramos relações entre os coeficientes $\lambda_{10}, \lambda_{01}$ e $\lambda_{11}$ correspondentes aos comportamentos geométricos das marginais. Os resultados são resumidos na Tabela 3.2.3, que por sua vez contém a Tabela 3.2.1.

\section{Tabela 3.2.3}

Condições encontradas para os coeficientes $\lambda_{10}, \lambda_{01}$ e $\lambda_{11}$ dadas as diferentes marginais de $h(x, y)=\exp \left(\lambda_{0}+\lambda_{10} x+\lambda_{01} y+\lambda_{11} x y\right)$, para $\left.x_{0}, y_{0} \in\right] a, b[$.

\begin{tabular}{|c|c|c|c|}
\hline & $g_{Y}^{i}$ & $g_{Y}^{d}$ & $g_{Y}^{m}$ \\
\hline \hline & $L\left(a, \lambda_{01} ; \lambda_{11}\right) \leq \lambda_{10}$ & $L\left(a, \lambda_{01} ; \lambda_{11}\right) \leq \lambda_{10}$ & $L\left(a, \lambda_{01} ; \lambda_{11}\right) \leq \lambda_{10}$ \\
$f_{X}^{i}$ & $\mathrm{e}$ & $\mathrm{e}$ & $\mathrm{e}$ \\
& $L\left(a, \lambda_{10} ; \lambda_{11}\right) \leq \lambda_{01}$ & $L\left(b, \lambda_{10} ; \lambda_{11}\right) \geq \lambda_{01}$ & $L\left(y_{0}, \lambda_{10} ; \lambda_{11}\right)=\lambda_{01}$ \\
\hline & $L\left(b, \lambda_{01} ; \lambda_{11}\right) \geq \lambda_{10}$ & $L\left(b, \lambda_{01} ; \lambda_{11}\right) \geq \lambda_{10}$ & $L\left(b, \lambda_{01} ; \lambda_{11}\right) \geq \lambda_{10}$ \\
$f_{X}^{d}$ & $\mathrm{e}$ & $\mathrm{e}$ & $\mathrm{e}$ \\
& $L\left(a, \lambda_{10} ; \lambda_{11}\right) \leq \lambda_{01}$ & $L\left(b, \lambda_{10} ; \lambda_{11}\right) \geq \lambda_{01}$ & $L\left(y_{0}, \lambda_{10} ; \lambda_{11}\right)=\lambda_{01}$ \\
\hline & $L\left(x_{0}, \lambda_{01} ; \lambda_{11}\right)=\lambda_{10}$ & $\boldsymbol{L}\left(\boldsymbol{x}_{0}, \boldsymbol{\lambda}_{01} ; \boldsymbol{\lambda}_{11}\right)=\boldsymbol{\lambda}_{10}$ & $L\left(x_{0}, \lambda_{01} ; \lambda_{11}\right)=\lambda_{10}$ \\
$f_{X}^{m}$ & $\mathrm{e}$ & $\mathrm{e}$ & $\mathrm{e}$ \\
& $L\left(a, \lambda_{10} ; \lambda_{11}\right) \leq \lambda_{01}$ & $\boldsymbol{L}\left(\boldsymbol{b}, \boldsymbol{\lambda}_{10} ; \boldsymbol{\lambda}_{11}\right) \geq \boldsymbol{\lambda}_{01}$ & $L\left(y_{0}, \lambda_{10} ; \lambda_{11}\right)=\lambda_{01}$ \\
\hline
\end{tabular}

Pode ser visto na Tabela 3.2.3 que, quando $f_{X}$ possui um mínimo em $x_{0}$ e $g_{Y}$ é decrescente, ou seja $f_{X}^{m}$ e $g_{Y}^{d}$, os coeficientes $\lambda_{10}, \lambda_{01}$ e $\lambda_{11}$ devem satisfazer as seguintes relações

$$
L\left(x_{0}, \lambda_{01} ; \lambda_{11}\right)=\lambda_{10} \quad \text { e } \quad L\left(b, \lambda_{10} ; \lambda_{11}\right) \geq \lambda_{01} .
$$


A seguir consideramos $\lambda_{11}$ um número real fixo não nulo. Com o intuito de mostrar graficamente as regiões do plano $\left(\lambda_{10}, \lambda_{01}\right)$ correspondentes aos comportamentos geométricos possíveis das marginais, enunciamos as seguintes propriedades da função $L$.

Proposição 3.2.6. Sejam $\lambda, \lambda_{11} \in \mathbb{R} \operatorname{com} \lambda_{11} \neq 0$.

1. $L\left(a, \lambda+(b-a) \lambda_{11} ; \lambda_{11}\right)=L\left(b, \lambda ; \lambda_{11}\right)$;

2. Existem números reais $\lambda_{a}^{0}$ e $\lambda_{b}^{0}$ tais que $L\left(a, \lambda_{a}^{0} ; \lambda_{11}\right)=\lambda_{a}^{0}$ e $L\left(b, \lambda_{b}^{0} ; \lambda_{11}\right)=\lambda_{b}^{0}$, sendo $\lambda_{a}^{0}$ e $\lambda_{b}^{0}$ os únicos valores que satisfazem essas igualdades;

3. Existem números reais $\lambda_{a}^{*}$ e $\lambda_{b}^{*}$ tais que $L\left(a, \lambda_{b}^{*} ; \lambda_{11}\right)=\lambda_{a}^{*}$ e $L\left(b, \lambda_{a}^{*} ; \lambda_{11}\right)=\lambda_{b}^{*}$, sendo $\lambda_{a}^{*}$ $e \lambda_{b}^{*}$ os únicos valores que satisfazem essas igualdades.

Prova: Ver a demonstração no Apêndice C.

A proposição anterior é bastante útil na determinação das regiões do plano $\left(\lambda_{10}, \lambda_{01}\right)$ segundo as diferentes densidades marginais. Isto pode ser visto no seguinte corolário.

Corolário 3.2.7. Seja $\lambda_{11} \in \mathbb{R}, \lambda_{11} \neq 0$.

1. Os gráficos $\left(\lambda_{10}, L\left(a, \lambda_{10} ; \lambda_{11}\right)\right)$ e $\left(L\left(a, \lambda_{01} ; \lambda_{11}\right), \lambda_{01}\right)$ se cruzam unicamente no ponto $\left(\lambda_{a}^{0}, \lambda_{a}^{0}\right)$

2. Os gráficos $\left(\lambda_{10}, L\left(b, \lambda_{10} ; \lambda_{11}\right)\right)$ e $\left(L\left(b, \lambda_{01} ; \lambda_{11}\right), \lambda_{01}\right)$ se cruzam unicamente no ponto $\left(\lambda_{b}^{0}, \lambda_{b}^{0}\right)$;

3. Os gráficos $\left(\lambda_{10}, L\left(a, \lambda_{10} ; \lambda_{11}\right)\right)$ e $\left(L\left(b, \lambda_{01} ; \lambda_{11}\right), \lambda_{01}\right)$ se cruzam unicamente no ponto $\left(\lambda_{b}^{*}, \lambda_{a}^{*}\right)$

4. Os gráficos $\left(\lambda_{10}, L\left(b, \lambda_{10} ; \lambda_{11}\right)\right)$ e $\left(L\left(a, \lambda_{01} ; \lambda_{11}\right), \lambda_{01}\right)$ se cruzam unicamente no ponto $\left(\lambda_{a}^{*}, \lambda_{b}^{*}\right)$.

Prova: A prova é conseqüência imediata da Proposição 3.2.6.

Outra conseqüência da Proposição 3.2.6, que auxilia na especificação das diferentes regiões do plano $\left(\lambda_{10}, \lambda_{01}\right)$, é dada no corolário a seguir. 
Corolário 3.2.8. As quantidades definidas na Proposição 3.2.6 satisfazem as seguintes desigualdades

$$
\begin{cases}-b \lambda_{11}<\lambda_{b}^{*}<\lambda_{b}^{0}<\lambda_{a}^{0}<\lambda_{a}^{*}<-a \lambda_{11}, & \text { se } \lambda_{11}>0 \\ -a \lambda_{11}<\lambda_{b}^{0}<\lambda_{b}^{*}<\lambda_{a}^{*}<\lambda_{a}^{0}<-b \lambda_{11}, & \text { se } \lambda_{11}<0 .\end{cases}
$$

Prova: Ver a demonstração no Apêndice C.

Por exemplo, para $a=1, b=2$ e $\lambda_{11}=-1$ temos que

$$
\lambda_{a}^{0}=1,545, \quad \lambda_{a}^{*}=1,538, \quad \lambda_{b}^{*}=1,462 \quad \text { e } \quad \lambda_{b}^{0}=1,454
$$

Consideremos a seguir $\lambda_{11}>0$. Utilizando as relações dadas na Tabela 3.2.3, assim como os resultados apresentados na Proposição 3.2.6 e nos seus corolários, podemos concluir que

1. Se ambas marginais possuem um mínimo em $] a, b\left[\right.$ então $\lambda_{10}$ e $\lambda_{01}$ devem satisfazer $\min \left[\lambda_{b}^{0}, L\left(b, \lambda_{01} ; \lambda_{11}\right)\right]<\lambda_{10}<\max \left[\lambda_{a}^{0}, L\left(a, \lambda_{01} ; \lambda_{11}\right)\right] \quad$ e $\min \left[\lambda_{b}^{0}, L\left(b, \lambda_{10} ; \lambda_{11}\right)\right]<\lambda_{01}<\max \left[\lambda_{a}^{0}, L\left(a, \lambda_{10} ; \lambda_{11}\right)\right]$;

2. Se ambas marginais são funções decrescentes então $\lambda_{10}$ e $\lambda_{01}$ devem satisfazer $\lambda_{10} \leq \max \left[\lambda_{b}^{0}, L\left(b, \lambda_{01} ; \lambda_{11}\right)\right]$ e $\lambda_{01} \leq \max \left[\lambda_{b}^{0}, L\left(b, \lambda_{10} ; \lambda_{11}\right)\right] ;$

3. Se ambas marginais são funções crescentes então $\lambda_{10}$ e $\lambda_{01}$ devem satisfazer $\lambda_{10} \geq \min \left[\lambda_{a}^{0}, L\left(a, \lambda_{01} ; \lambda_{11}\right)\right]$ e $\lambda_{01} \geq \min \left[\lambda_{a}^{0}, L\left(a, \lambda_{10} ; \lambda_{11}\right)\right]$

4. Se $f_{X}$ é crescente e $g_{Y}$ é decrescente então $\lambda_{10}$ e $\lambda_{01}$ devem satisfazer $\lambda_{10} \geq \max \left[\lambda_{a}^{*}, L\left(a, \lambda_{01} ; \lambda_{11}\right)\right]$ e $\lambda_{01} \leq \min \left[\lambda_{b}^{*}, L\left(b, \lambda_{10} ; \lambda_{11}\right)\right] ;$

5. Se $g_{Y}$ é crescente e $f_{X}$ é decrescente então $\lambda_{10}$ e $\lambda_{01}$ devem satisfazer $\lambda_{10} \leq \min \left[\lambda_{b}^{*}, L\left(b, \lambda_{01} ; \lambda_{11}\right)\right]$ e $\lambda_{01} \geq \max \left[\lambda_{a}^{*}, L\left(a, \lambda_{10} ; \lambda_{11}\right)\right]$

6. Se $f_{X}$ possui um mínimo e $g_{Y}$ é crescente então $\lambda_{10}$ e $\lambda_{01}$ devem satisfazer $\min \left[\lambda_{b}^{*}, L\left(b, \lambda_{01} ; \lambda_{11}\right)\right]<\lambda_{10}<\min \left[\lambda_{a}^{0}, L\left(a, \lambda_{01} ; \lambda_{11}\right)\right]$ e $\lambda_{01} \geq \max \left[\lambda_{a}^{0}, L\left(a, \lambda_{10} ; \lambda_{11}\right)\right] ;$

7. Se $f_{X}$ possui um mínimo e $g_{Y}$ é decrescente então $\lambda_{10}$ e $\lambda_{01}$ devem satisfazer $\max \left[\lambda_{b}^{0}, L\left(b, \lambda_{01} ; \lambda_{11}\right)\right]<\lambda_{10}<\max \left[\lambda_{a}^{*}, L\left(a, \lambda_{01} ; \lambda_{11}\right)\right]$ e $\lambda_{01} \leq \min \left[\lambda_{b}^{0}, L\left(b, \lambda_{10} ; \lambda_{11}\right)\right] ;$ 
8. Se $f_{X}$ é crescente e $g_{Y}$ possui um mínimo então $\lambda_{10}$ e $\lambda_{01}$ devem satisfazer $\lambda_{10} \geq \max \left[\lambda_{a}^{0}, L\left(a, \lambda_{01} ; \lambda_{11}\right)\right] \mathrm{e} \min \left[\lambda_{b}^{*}, L\left(b, \lambda_{10} ; \lambda_{11}\right)\right]<\lambda_{01}<\min \left[\lambda_{a}^{0}, L\left(a, \lambda_{10} ; \lambda_{11}\right)\right] ;$

9. Se $f_{X}$ é decrescente e $g_{Y}$ possui um mínimo então $\lambda_{10}$ e $\lambda_{01}$ devem satisfazer $\lambda_{10} \leq \min \left[\lambda_{b}^{0}, L\left(b, \lambda_{01} ; \lambda_{11}\right)\right]$ e $\max \left[\lambda_{b}^{0}, L\left(b, \lambda_{10} ; \lambda_{11}\right)\right]<\lambda_{01}<\max \left[\lambda_{a}^{*}, L\left(a, \lambda_{10} ; \lambda_{11}\right)\right]$.

Os casos de 1 até 9 listados servem como uma classificação para $\lambda_{11}>0$ fixo, a qual é resumida na Figura 3.2.4.

Figura 3.2.4

Regiões do plano $\left(\lambda_{10}, \lambda_{01}\right)$ correspondentes aos diferentes tipos de densidades marginais para $\lambda_{11}>0$ fixo quando $h(x, y)=\exp \left(\lambda_{0}+\lambda_{10} x+\lambda_{01} y+\lambda_{11} x y\right)$.

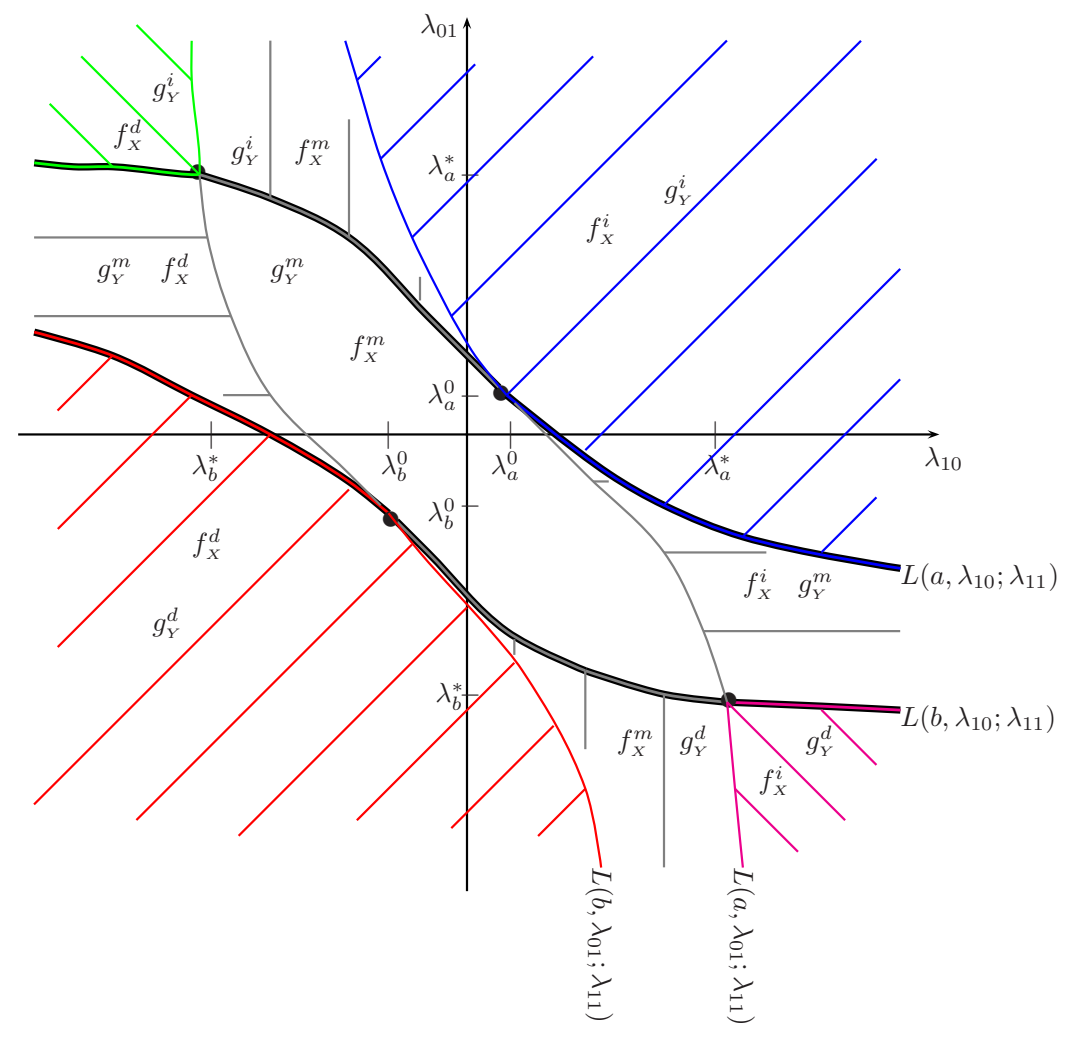


A seguir, na Tabela 3.2.5, apresentamos exemplos de coeficientes calculados usando como guia a Figura 3.2.4. Sejam $a=1, b=2$ e $\lambda_{11}=1$, então obtemos

$$
\lambda_{a}^{*}=-1,455, \quad \lambda_{a}^{0}=-1,462, \quad \lambda_{b}^{0}=-1,538 \quad \text { e } \quad \lambda_{b}^{*}=-1,545 .
$$

Tabela 3.2.5

Possíveis valores dos coeficientes para diferentes marginais de $h(x, y)=\exp \left(\lambda_{0}+\lambda_{10} x+\lambda_{01} y+\lambda_{11} x y\right),(x, y) \in[1,2] \times[1,2]$.

\begin{tabular}{|c|c|c|c|c|c|}
\hline$f_{X}$ & $g_{Y}$ & $\lambda_{11}$ & $\lambda_{10}$ & $\lambda_{01}$ & $\lambda_{0}$ \\
\hline \hline$f_{X}^{m}$ & $g_{Y}^{m}$ & 1 & $-1,5$ & $-1,5$ & 2,879 \\
\hline$f_{X}^{d}$ & $g_{Y}^{i}$ & 1 & -2 & 1 & $-1,005$ \\
\hline$f_{X}^{d}$ & $g_{Y}^{d}$ & 1 & -2 & -2 & 3,724 \\
\hline$f_{X}^{i}$ & $g_{Y}^{m}$ & 1 & -1 & $-1,54$ & 1,678 \\
\hline
\end{tabular}

Para $\lambda_{11}<0$ pode ser realizado um estudo equivalente obtendo uma divisão do plano $\left(\lambda_{10}, \lambda_{01}\right)$ similar à dada na Figura 3.2.4.

Um exemplo interessante é dado na Figura 3.2.6, na qual fornecemos uma representação para $h$ em $[1,2] \times[1,2]$ quando $f_{X}$ é decrescente e $g_{Y}$ é unimodal com moda em $y=1,5$. Conforme o observado no fim de $\S 2.1$ devemos considerar

$$
h=M-\tilde{h}=M-\exp \left(\lambda_{0}+\lambda_{10} x+\lambda_{01} y+\lambda_{11} x y\right) .
$$

Os coeficientes $\lambda_{10}, \lambda_{01}$ e $\lambda_{11}$ são escolhidos de tal forma que $\tilde{f}_{X}$ (a densidade marginal de $\tilde{h}$ da variável $X$ ) seja crescente e $\tilde{g_{Y}}$ (a densidade marginal de $\tilde{h}$ da variável $Y$ ) tenha um mínimo em $y=1,5$. Em particular escolhimos $M=\frac{2}{(b-a)^{2}}=2$. 
Figura 3.2.6

Densidade $h(x, y)=2-\exp (1,546-x-1,54 y+x y)$ para $(x, y) \in[1,2] \times[1,2]$.

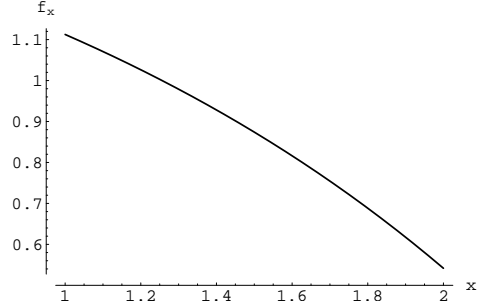

(a) Densidade marginal $f_{X}$.

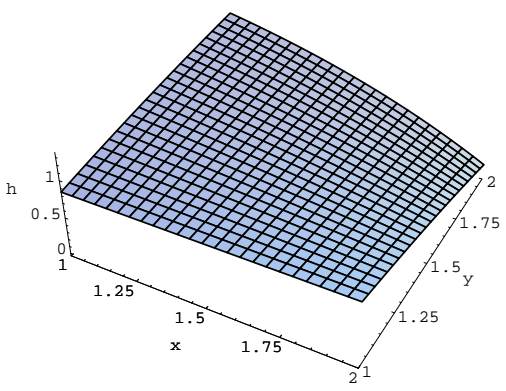

(c) Densidade conjunta $h$.

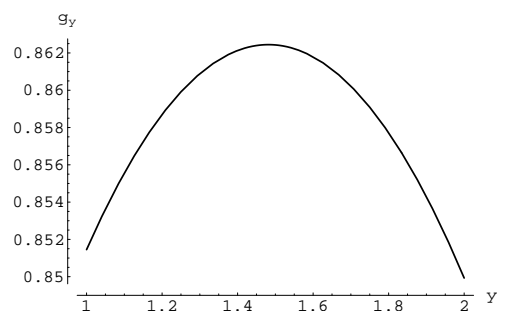

(b) Densidade marginal $g_{Y}$.

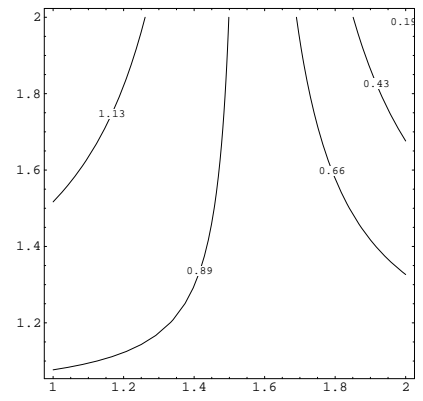

(d) Curvas de nível da densidade conjunta.

\subsubsection{Família de quatro parâmetros}

Aqui estudamos a densidade dada por

$$
h(x, y)=\exp \left(\lambda_{0}+\lambda_{10} x+\lambda_{01} y+\lambda_{11} x y+\lambda_{20} x^{2}\right), \quad(x, y) \in[a, b] \times[a, b] .
$$

Sendo as correspondentes marginais

$$
f_{X}(x)= \begin{cases}\exp \left(\lambda_{0}+\lambda_{10} x+\lambda_{20} x^{2}\right)\left(\frac{\exp \left(\left(\lambda_{01}+\lambda_{11} x\right) b\right)-\exp \left(\left(\lambda_{01}+\lambda_{11} x\right) a\right)}{\lambda_{01}+\lambda_{11} x}\right), & \text { se } \lambda_{01}+\lambda_{11} x \neq 0 \\ \exp \left(\lambda_{0}+\lambda_{10} x+\lambda_{20} x^{2}\right)(b-a), & \text { se } \lambda_{01}+\lambda_{11} x=0\end{cases}
$$

$\mathrm{e}$

$$
g_{Y}(y)=\exp \left(\lambda_{0}+\lambda_{01} y\right) \int_{a}^{b} \exp \left(\left(\lambda_{10}+\lambda_{11} y\right) x+\lambda_{20} x^{2}\right) d x
$$


Claramente, a análise de $f_{X}$ é diferente da análise de $g_{Y}$. Antes de continuar salientamos que os resultados apresentados nesta seção resolvem, por simetria, o caso no qual a densidade é $h(x, y)=\exp \left(\lambda_{0}+\lambda_{10} x+\lambda_{01} y+\lambda_{11} x y+\lambda_{02} y^{2}\right)$.

\section{Propriedades de $f_{X}$}

Começamos o estudo da $f_{X}$ com a seguinte proposição que fornece possíveis valores para os coeficientes quando a densidade é crescente ou decrescente.

Proposição 3.2.9. Seja $P_{f}(x, y)=\lambda_{10}+\lambda_{11} y+2 \lambda_{20} x$.

1. Se $\min _{(x, y) \in[a, b] \times[a, b]} P_{f}(x, y) \geq 0$, então $f_{X}$ é uma função crescente;

2. Se $\max _{(x, y) \in[a, b] \times[a, b]} P_{f}(x, y) \leq 0$, então $f_{X}$ é uma função decrescente.

Prova: Ver a demonstração no Apêndice C.

Os possíveis minimizadores e maximizadores do polinômio $P_{f}$ são mostrados nos seguintes dois corolários.

Corolário 3.2.10. $f_{X}$ é uma função crescente se alguma das seguintes condições é satisfeita.

1. $\lambda_{20}>0, \lambda_{11} \geq 0$ e $P_{f}(a, a) \geq 0$;

2. $\lambda_{20}>0, \lambda_{11}<0$ e $P_{f}(a, b) \geq 0$;

3. $\lambda_{20}<0, \lambda_{11} \geq 0$ e $P_{f}(b, a) \geq 0$;

4. $\lambda_{20}<0, \lambda_{11}<0$ e $P_{f}(b, b) \geq 0$.

Prova: Ver a demonstração no Apêndice C. 
Corolário 3.2.11. $f_{X}$ é uma função decrescente se alguma das seguintes condições é satisfeita.

1. $\lambda_{20}>0, \lambda_{11} \geq 0$ e $P_{f}(b, b) \leq 0$;

2. $\lambda_{20}>0, \lambda_{11}<0$ e $P_{f}(b, a) \leq 0$;

3. $\lambda_{20}<0, \lambda_{11} \geq 0$ e $P_{f}(a, b) \leq 0$;

4. $\lambda_{20}<0, \lambda_{11}<0$ e $P_{f}(a, a) \leq 0$.

Prova: Demonstração análoga ao corolário anterior, Corolário 3.2.10.

Os dois corolários prévios servem como guia para determinar possíveis coeficientes $\lambda_{10}, \lambda_{11}$ e $\lambda_{20}$ segundo a natureza crescente ou decrescente da marginal $f_{X}$. Na Tabela 3.2 .7 mostramos valores dos coeficientes obtidos aplicando tais corolários. Observamos antes, que as relações dadas não fornecem condições no coeficiente $\lambda_{01}$ do termo $y$.

\section{Tabela 3.2 .7}

Possíveis valores dos coeficientes $\lambda_{10}, \lambda_{11}$ e $\lambda_{20}$ correspondentes a marginais $f_{X}$ crescentes ou decrescentes, quando $h(x, y)=\exp \left(\lambda_{0}+\lambda_{10} x+\lambda_{01} y+\lambda_{11} x y+\lambda_{20} x^{2}\right)$ para $(x, y) \in[-1,1] \times[-1,1]$.

\begin{tabular}{|c||c|c|c|}
\hline$f_{X}$ & $\lambda_{10}$ & $\lambda_{11}$ & $\lambda_{20}$ \\
\hline \hline \multirow{3}{*}{$f_{X}^{i}$} & $\mathbf{4}$ & $\mathbf{1}$ & $\mathbf{1}$ \\
\cline { 2 - 4 } & 6 & -1 & 1 \\
\cline { 2 - 4 } & 5 & -1 & -1 \\
\hline \multirow{3}{*}{$f_{X}^{d}$} & -5 & 1 & 1 \\
\cline { 2 - 4 } & -6 & 2 & 1 \\
\cline { 2 - 4 } & -4 & 2 & -1 \\
\hline
\end{tabular}

Por exemplo, a primeira linha da Tabela 3.2.7 foi construída utilizando o Corolário 3.2.10 (item 1). Isto significa que, quando $f_{X}$ é crescente em $[-1,1]$, os valores $\lambda_{10}=4, \lambda_{11}=1 \mathrm{e}$ $\lambda_{20}=1$ definem uma possível representação de $h$, já que satisfazem as condições

$$
\lambda_{20}>0, \quad \lambda_{11} \geq 0 \quad \text { e } \quad P_{f}(a, a) \geq 0
$$


A seguir damos algumas propriedades de $f_{X}$ para quando $\lambda_{11}=0$. Salientamos que nesse caso as variáveis aleatórias $X$ e $Y$ são independentes. É oportuno lembrar que $x_{0}$ é um ponto crítico de $f_{X}$ se $\left.\frac{d f_{X}}{d x}(x)\right|_{x=x_{0}}=0$. Um ponto crítico pode ser um minimizador, um maximizador ou um ponto no qual muda a concavidade da função, ou seja, ponto de inflexão.

Proposição 3.2.12. Se $\lambda_{11}=0$ e $\left.x_{0}=\frac{-\lambda_{10}}{2 \lambda_{20}} \in\right] a, b\left[\right.$ então $f_{X}$ possui um único ponto crítico em $x_{0}$.

Prova: Ver a demonstração no Apêndice C.

Proposição 3.2.13. Se $\left.\lambda_{11}=0, x_{0}=\frac{-\lambda_{10}}{2 \lambda_{20}} \in\right] a, b\left[\right.$ e $\lambda_{20}<0$ então $f_{X}$ é unimodal com moda em $x_{0}$.

Prova: Ver a demonstração no Apêndice C.

Proposição 3.2.14. Se $\lambda_{11}=0$ e $x_{0}=\frac{-\lambda_{10}}{2 \lambda_{20}}=\frac{a+b}{2}$ então $f_{X}$ é simétrica.

Prova: Ver a demonstração no Apêndice C.

O seguintes resultados, Lemma 3.2.15 e Proposição 3.2.16, são válidos para $\lambda_{20}>0 \mathrm{e}$ caracterizam, por meio da função $L$ definida em (3.6), os posíveis comportamentos de $f_{X}$, isto é $f_{X}^{i}, f_{X}^{d}$ e $f_{X}^{m}$.

Lema 3.2.15. Se $\lambda_{20}>0$, então $f_{X}$ é uma função estritamente convexa.

Prova: Ver a demonstração no Apêndice C.

Proposição 3.2.16. Seja $\lambda_{20}>0$.

1. $f_{X}$ é crescente se, e somente se, $\lambda_{10} \geq-2 \lambda_{20} a+L\left(a, \lambda_{01} ; \lambda_{11}\right)$;

2. $f_{X}$ é decrescente se, e somente se, $\lambda_{10} \leq-2 \lambda_{20} b+L\left(b, \lambda_{01} ; \lambda_{11}\right)$;

3. $f_{X}$ possui um ponto mínimo se, e somente se, $-2 \lambda_{20} b+L\left(b, \lambda_{01} ; \lambda_{11}\right)<\lambda_{10} e$ $\lambda_{10}<-2 \lambda_{20} a+L\left(a, \lambda_{01} ; \lambda_{11}\right)$.

Prova: Ver a demonstração no Apêndice C. 
Corolário 3.2.17. Se $x_{0}$ for um ponto crítico de $f_{X}$, então $\lambda_{10}+2 \lambda_{20} x_{0}-L\left(x_{0}, \lambda_{01} ; \lambda_{11}\right)=0$.

Prova: Conseqüência da expressão da derivada de $f_{X}$ obtida na prova da Proposição 3.2.16.

A partir da caracterização fornecida na Proposição 3.2.16 podem ser obtidas relações mais simples entre os coeficientes no caso em que $\lambda_{20}$ é positivo, a seguir apresentamos tais relações.

Corolário 3.2.18. Seja $\lambda_{20}>0$.

1. Se $\lambda_{11}>0, \lambda_{01}+\lambda_{11} a>0$ e $-2 \lambda_{20} a-\lambda_{11}\left(\frac{b+a}{2}\right) \leq \lambda_{10}$ ou se $\lambda_{11}<0, \lambda_{01}+\lambda_{11} b>0$ e $-2 \lambda_{20} b-\lambda_{11}\left(\frac{b+a}{2}\right) \geq \lambda_{10}$ então $f_{X}$ é crescente;

2. Se $\lambda_{11}>0, \lambda_{01}+\lambda_{11} b<0$ e $-2 \lambda_{20} b-\lambda_{11}\left(\frac{b+a}{2}\right) \geq \lambda_{10}$ ou se $\lambda_{11}<0, \lambda_{01}+\lambda_{11} a<0 e$ $-2 \lambda_{20} a-\lambda_{11}\left(\frac{b+a}{2}\right) \leq \lambda_{10}$, então $f_{X}$ é decrescente;

3. Se $\lambda_{11}>0, \lambda_{01}+\lambda_{11} a \leq 0 \leq \lambda_{01}+\lambda_{11} b e-2 \lambda_{20} b-\lambda_{11}\left(\frac{b+a}{2}\right)<\lambda_{10}<-2 \lambda_{20} a-\lambda_{11}\left(\frac{b+a}{2}\right)$ ou se $\lambda_{11}<0, \lambda_{01}+\lambda_{11} b<0<\lambda_{01}+\lambda_{11} a e-2 \lambda_{20} b-\lambda_{11}\left(\frac{b+a}{2}\right)<\lambda_{10}<-2 \lambda_{20} a-\lambda_{11}\left(\frac{b+a}{2}\right)$, então $f_{X}$ possui um ponto mínimo.

Prova: Ver a demonstração no Apêndice C.

Na seguinte proposição analisamos o caso em que $\lambda_{20}<0$.

Proposição 3.2.19. Seja $\lambda_{20}<0$.

1. Se $\lambda_{11}>0$ então

(a) $\frac{-\lambda_{11} b-\lambda_{10}}{2 \lambda_{20}} \leq$ a implica que $f_{X}$ é decrescente;

(b) $a<\frac{-\lambda_{11} a-\lambda_{10}}{2 \lambda_{20}}<\frac{-\lambda_{11} b-\lambda_{10}}{2 \lambda_{20}}<b$ implica que $f_{X}$ possui pelo menos um ponto crítico;

(c) $b \leq \frac{-\lambda_{11} a-\lambda_{10}}{2 \lambda_{20}}$ implica que $f_{X}$ é crescente.

2. Se $\lambda_{11}<0$ então

(a) $\frac{-\lambda_{11} a-\lambda_{10}}{2 \lambda_{20}} \leq$ a implica que $f_{X}$ é decrescente;

(b) $a<\frac{-\lambda_{11} b-\lambda_{10}}{2 \lambda_{20}}<\frac{-\lambda_{11} a-\lambda_{10}}{2 \lambda_{20}}<b$ implica que $f_{X}$ possui pelo menos um ponto crítico;

(c) $b \leq \frac{-\lambda_{11} b-\lambda_{10}}{2 \lambda_{20}}$ implica que $f_{X}$ é crescente. 
Prova: Ver a demonstração no Apêndice C.

Para alguns valores negativos de $\lambda_{20}$ é possível dar uma caracterização similar à dada na Proposição 3.2.16.

Proposição 3.2.20. Seja $\lambda_{20}<-\frac{\lambda_{11}^{2}(b-a)^{2}}{24}$.

1. $f_{X}$ é crescente se, e somente se, $\lambda_{10} \geq-2 \lambda_{20} b+L\left(b, \lambda_{01} ; \lambda_{11}\right)$;

2. $f_{X}$ é decrescente se, e somente se, $\lambda_{10} \leq-2 \lambda_{20} a+L\left(a, \lambda_{01} ; \lambda_{11}\right)$;

3. $f_{X}$ é unimodal se, e somente se, $-2 \lambda_{20} a+L\left(a, \lambda_{01} ; \lambda_{11}\right)<\lambda_{10}<-2 \lambda_{20} b+L\left(b, \lambda_{01} ; \lambda_{11}\right)$.

Prova: Ver a demonstração no Apêndice C.

\section{Propriedades de $g_{Y}$}

Aqui estudamos a influência do comportamento da marginal $g_{Y}$ nos coeficientes da densidade conjunta $h$. Lembramos que neste caso a marginal $g_{Y}$ está dada por

$$
g_{Y}(y)=\exp \left(\lambda_{0}+\lambda_{01} y\right) \int_{a}^{b} \exp \left(\left(\lambda_{10}+\lambda_{11} y\right) x+\lambda_{20} x^{2}\right) d x .
$$

Observemos que se $\lambda_{10}=\lambda_{11}=0$, então as variáveis $X$ e $Y$ são independentes e $g_{Y}$ é constante. Esse caso resulta ser de pouco interesse e por isso vamos assumir que algum de tais coeficientes é não nulo. Assim como no caso da marginal $f_{X}$, começamos apresentando a seguinte proposição que fornece condições para alguns dos coeficientes segundo a natureza crescente ou decrescente de $g_{Y}$.

Proposição 3.2.21. Seja $P_{g}(x, 0)=\lambda_{01}+\lambda_{11} x$, onde $\lambda_{01} \neq 0$ ou $\lambda_{11} \neq 0$.

1. Se $\min _{x \in[a, b]} P_{g}(x, 0) \geq 0$, então $g_{Y}$ é uma função crescente;

2. Se $\max _{x \in[a, b]} P_{g}(x, 0) \leq 0$, então $g_{Y}$ é uma função decrescente.

Prova: Ver a demonstração no Apêndice C.

Nos seguintes dois corolários são exibidos os possíveis minimizadores e maximizadores do polinômio $P_{g}(\cdot, 0)$. 
Corolário 3.2.22. $g_{Y}$ é uma função crescente se alguma das seguintes condições é satisfeita.

1. $\lambda_{11}>0$ e $P_{g}(a, 0) \geq 0$;

2. $\lambda_{11}<0$ e $P_{g}(b, 0) \geq 0$;

3. $\lambda_{11}=0$ e $\lambda_{01}>0$.

Prova: Conseqüência da Proposição 3.2.21 e da definição de $P_{g}(\cdot, 0)$.

Corolário 3.2.23. $g_{Y}$ é uma função decrescente se alguma das seguintes condições é satisfeita.

1. $\lambda_{11}>0$ e $P_{g}(b, 0) \leq 0$;

2. $\lambda_{11}<0$ e $P_{g}(a, 0) \leq 0$;

3. $\lambda_{11}=0$ e $\lambda_{01}<0$.

Prova: Conseqüência imediata da Proposição 3.2 .21 e da definição de $P_{g}(\cdot, 0)$.

A seguinte proposição enuncia a convexidade de $g_{Y}$.

Proposição 3.2.24. A densidade marginal $g_{Y}$ é uma função convexa. A convexidade é estrita para $\lambda_{01} \neq 0$ ou $\lambda_{11} \neq 0$.

Prova: Ver a demonstração no Apêndice C.

Corolário 3.2.25. Se $g_{Y}$ possui um ponto mínimo, então $\left.-\frac{\lambda_{01}}{\lambda_{11}} \in\right] a, b[$.

Prova: Segue das Proposições 3.2.21 e 3.2.24.

Finalizamos o estudo do presente caso com a Figura 3.2.8, na qual exemplificamos uma possível representação da densidade conjunta $h$ de domínio $[-1,1] \times[-1,1]$ para $f_{X}$ unimodal (moda $\left.x_{0}=-0,535\right)$ e $g_{Y}$ crescente. Os valores dos coeficientes foram escolhidos segundo a Proposição 3.2.20 (item 3) e o Corolário 3.2.22 (item 1). 
Figura 3.2.8

Densidade $h(x, y)=\exp \left(-1,832-1,5+2 y+x y-x^{2}\right)$ para $(x, y) \in[-1,1] \times[-1,1]$.

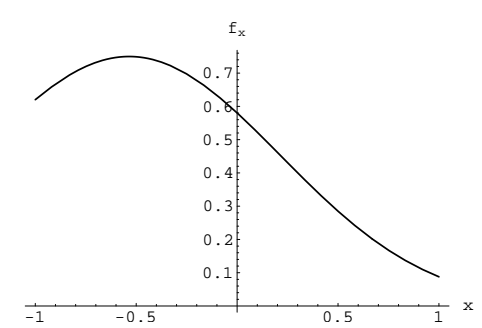

(a) Densidade marginal $f_{X}$.

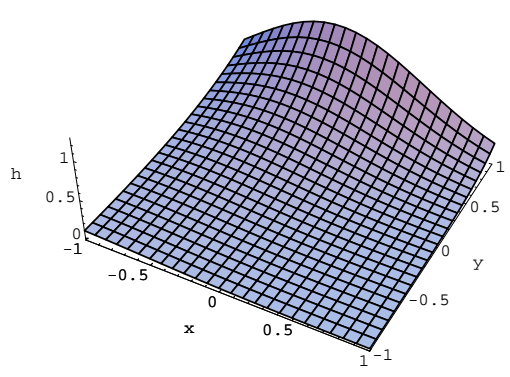

(c) Densidade conjunta $h$.

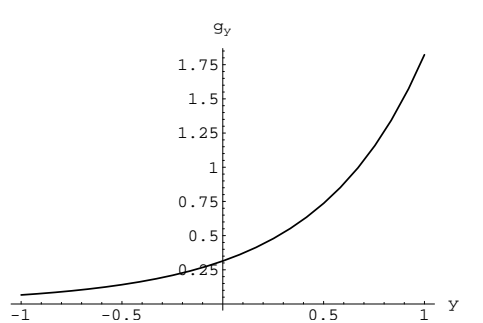

(b) Densidade marginal $g_{Y}$.

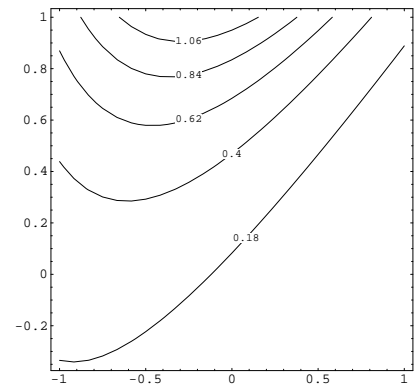

(d) Curvas de nível da densidade conjunta.

\subsubsection{Família de cinco parâmetros}

Aqui analisamos a relação dada pela equação (3.1), ou seja,

$$
h(x, y)=\exp \left(\lambda_{0}+\lambda_{10} x+\lambda_{01} y+\lambda_{11} x y+\lambda_{20} x^{2}+\lambda_{02} y^{2}\right)
$$

para $(x, y) \in[a, b] \times[a, b], \lambda_{20} \neq 0$ e $\lambda_{02} \neq 0$. As marginais correspondentes são

$$
f_{X}(x)=\exp \left(\lambda_{0}+\lambda_{10} x+\lambda_{20} x^{2}\right) \int_{a}^{b} \exp \left(\lambda_{01} y+\lambda_{11} x y+\lambda_{02} y^{2}\right) d y
$$

e

$$
g_{Y}(y)=\exp \left(\lambda_{0}+\lambda_{01} y+\lambda_{02} y^{2}\right) \int_{a}^{b} \exp \left(\lambda_{10} x+\lambda_{11} x y+\lambda_{20} x^{2}\right) d x
$$


Neste caso, pelas expressões das densidades, vemos que o estudo de uma é facilmente adaptado à outra. Por exemplo, para a densidade $f_{X}$ é válida a Proposição 3.2.9 e os Corolários 3.2.10 e 3.2.11 e tais resultados podem ser modificados para a densidade $g_{Y}$. Isto é realizado mudando $\lambda_{10}$ por $\lambda_{01}, \lambda_{20}$ por $\lambda_{02}$ e $x$ por $y$ como mostra a seguinte proposição e os dois corolários correspodentes.

Proposição 3.2.26. Seja $P_{g}(x, y)=\lambda_{01}+\lambda_{11} x+2 \lambda_{02} y$.

1. Se $\min _{(x, y) \in[a, b] \times[a, b]} P_{g}(x, y) \geq 0$, então $g_{Y}$ é uma função crescente;

2. Se $\max _{(x, y) \in[a, b] \times[a, b]} P_{g}(x, y) \leq 0$, então $g_{Y}$ é uma função decrescente.

Prova: Prova análoga à demonstração da Proposição 3.2.9.

Corolário 3.2.27. $g_{Y}$ é uma função crescente se alguma das seguintes condições é satisfeita.

1. $\lambda_{02}>0, \lambda_{11} \geq 0$ e $P_{g}(a, a) \geq 0$;

2. $\lambda_{02}>0, \lambda_{11}<0$ e $P_{g}(b, a) \geq 0$;

3. $\lambda_{02}<0, \lambda_{11} \geq 0$ e $P_{g}(a, b) \geq 0$;

4. $\lambda_{02}<0, \lambda_{11}<0$ e $P_{g}(b, b) \geq 0$.

Prova: Demonstração similar à prova do Corolário 3.2.10.

Corolário 3.2.28. $g_{Y}$ é uma função decrescente se alguma das seguintes condições é satisfeita.

1. $\lambda_{02}>0, \lambda_{11} \geq 0$ e $P_{g}(b, b) \leq 0$;

2. $\lambda_{02}>0, \lambda_{11}<0$ e $P_{g}(a, b) \leq 0$;

3. $\lambda_{02}<0, \lambda_{11} \geq 0$ e $P_{g}(b, a) \leq 0$;

4. $\lambda_{02}<0, \lambda_{11}<0$ e $P_{g}(a, a) \leq 0$. 
Prova: Demonstração similar à prova do Corolário 3.2.10.

A combinação das Proposições 3.2.9 e 3.2.26 permite obter os seguintes resultados para o caso específico em que $\lambda_{20}=\lambda_{02}$.

Corolário 3.2.29. Sejam $\lambda_{20}, \lambda_{02} \in \mathbb{R}$ e $\lambda_{20}=\lambda_{02} \neq 0$.

1. Se $\lambda_{10} \geq \lambda_{01} e \min _{(x, y) \in[a, b] \times[a, b]} P_{g}(x, y) \geq 0$, então $f_{X}$ e $g_{Y}$ são funções crescentes;

2. Se $\lambda_{01} \geq \lambda_{10} e \min _{(x, y) \in[a, b] \times[a, b]} P_{f}(x, y) \geq 0$, então $f_{X}$ e $g_{Y}$ são funções crescentes;

3. Se $\lambda_{10} \geq \lambda_{01} e \max _{(x, y) \in[a, b] \times[a, b]} P_{f}(x, y) \leq 0$, então $f_{X}$ e $g_{Y}$ são funções decrescentes;

4. Se $\lambda_{01} \geq \lambda_{10} e \max _{(x, y) \in[a, b] \times[a, b]} P_{g}(x, y) \leq 0$, então $f_{X}$ e $g_{Y}$ são funções decrescentes.

Prova: Ver a demonstração no Apêndice C.

Se considerarmos o caso especial em que $a=-b$ e os coeficientes de $x$ e $y$ são nulos, ambas densidades serão simétricas, isto pode ser visto na seguinte proposição.

Proposição 3.2.30. Se $a=-b$ e $\lambda_{10}=\lambda_{01}=0$, então $f_{X}$ e $g_{Y}$ são funções simétricas.

Prova: Ver a demonstração no Apêndice C.

Incluindo nas hipóteses da proposição anterior relações entre os coeficientes e o extremo $b$ que garantam a negatividade da segunda derivada na origem, obtemos que uma das densidades também é unimodal. Na Proposição 3.2.31 são enunciadas tais relações, mas antes precisamos definir a função sinal como segue

$$
\operatorname{sgn}(z) \stackrel{\text { def }}{=} \begin{cases}1, & \text { se } z \geq 0 \\ -1, & \text { se } z<0\end{cases}
$$


Proposição 3.2.31. Sejam $a=-b, \lambda_{20}<0, \lambda_{02}<0$ e $\lambda_{10}=\lambda_{01}=0$.

1. Se $2 \lambda_{20}+\left(-2 \lambda_{20} b+\operatorname{sgn}\left(\lambda_{11}\right) \lambda_{11} b\right)^{2}<0$, então $f_{X}$ e $g_{Y}$ são funções simétricas e $f_{X}$ é unimodal;

2. Se $2 \lambda_{02}+\left(-2 \lambda_{02} b+\operatorname{sgn}\left(\lambda_{11}\right) \lambda_{11} b\right)^{2}<0$, então $f_{X}$ e $g_{Y}$ são funções simétricas e $g_{Y}$ é unimodal.

Prova: Ver a demonstração no Apêndice C.

No seguinte corolário mostramos os casos em que a origem é um minimizador.

Corolário 3.2.32. Sejam $a=-b$ e $\lambda_{10}=\lambda_{01}=0$.

1. Se $\lambda_{20}>0$, então $f_{X}$ e $g_{Y}$ são funções simétricas e $x_{0}=0$ é minimizador de $f_{X}$;

2. Se $\lambda_{02}>0$, então $f_{X}$ e $g_{Y}$ são funções simétricas e $y_{0}=0$ é minimizador de $g_{Y}$.

Prova: Segue da expressão da segunda derivada de $f_{X}$ e de $g_{Y}$ obtidas na prova da Proposição 3.2 .31 .

Finalizamos a análise deste caso apresentando na Figura 3.2.9 uma possível representação para a densidade $h$ quando ambas marginais são simétricas e unimodais no domínio $[-1,1]$. Os coeficientes deste exemplo foram escolhidos segundo a Proposição 3.2.31.

\subsection{Polinômio de grau três}

Nesta seção consideramos a relação dada por (3.2), ou seja,

$$
h(x, y)=\exp \left(\lambda_{0}+\lambda_{10} x+\lambda_{01} y+\lambda_{11} x y+\lambda_{21} x^{2} y\right),
$$

para $(x, y) \in[a, b] \times[a, b]$ e $\lambda_{21} \neq 0$. As marginais correspondentes são

$$
f_{X}(x)= \begin{cases}\frac{\exp \left(\lambda_{0}+\lambda_{10} x\right)\left(\exp \left(\left(\lambda_{01}+\lambda_{11} x+\lambda_{21} x^{2}\right) b\right)-\exp \left(\left(\lambda_{01}+\lambda_{11} x+\lambda_{21} x^{2}\right) a\right)\right)}{\lambda_{01}+\lambda_{11} x+\lambda_{21} x^{2}}, & \text { se } \lambda_{01}+\lambda_{11} x+\lambda_{21} x^{2} \neq 0 \\ \exp \left(\lambda_{0}+\lambda_{10} x\right)(b-a), & \text { se } \lambda_{01}+\lambda_{11} x+\lambda_{21} x^{2}=0\end{cases}
$$

e

$$
g_{Y}(y)=\exp \left(\lambda_{0}+\lambda_{01} y\right) \int_{a}^{b} \exp \left(\left(\lambda_{10}+\lambda_{11} y\right) x+\lambda_{21} y x^{2}\right) d x .
$$

Assim como na seção 3.2.2, as análises de $f_{X}$ e de $g_{Y}$ são diferentes. 
Figura 3.2.9

Densidade $h(x, y)=\exp \left(-1,243+0,1 x y-0,1 x^{2}-0,35 y^{2}\right)$, para $(x, y) \in[-1,1] \times[-1,1]$.

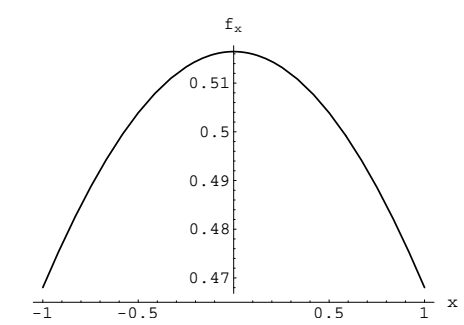

(a) Densidade marginal $f_{X}$.

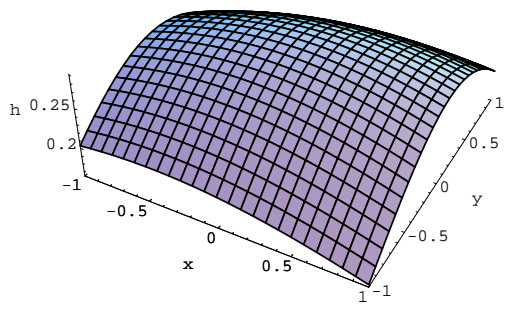

(c) Densidade conjunta $h$.

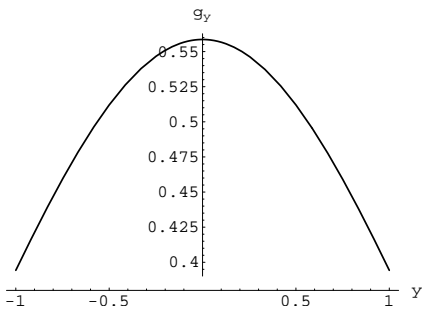

(b) Densidade marginal $g_{Y}$.

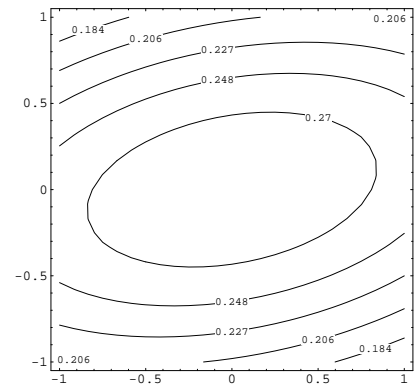

(d) Curvas de nível da densidade conjunta.

\section{Propriedades de $f_{X}$}

Começamos pesquisando as propriedades da marginal $f_{X}$. Na seguinte proposição damos condições para quando $f_{X}$ for crescente ou decrescente.

Proposição 3.3.1. Seja $P_{f}(x, y)=\lambda_{10}+\lambda_{11} y+2 \lambda_{21} x y$.

1. Se $\min _{(x, y) \in[a, b] \times[a, b]} P_{f}(x, y) \geq 0$, então $f_{X}$ é uma função crescente;

2. Se $\max _{(x, y) \in[a, b] \times[a, b]} P_{f}(x, y) \leq 0$, então $f_{X}$ é uma função decrescente.

Prova: A demonstração é análoga as provas das Proposições 3.2.9 e 3.2.21. 
Como conseqüência da proposição anterior obtemos os seguintes resultados, nos quais simplesmente mostramos os diferentes minimizadores e maximizadores.

Corolário 3.3.2. Sejam $x_{1}=\frac{-\lambda_{11}}{2 \lambda_{21}}$ e $x_{2}=\frac{-\lambda_{11}+2 \lambda_{21}(a+b)}{2 \lambda_{21}}$. Então, $f_{X}$ é uma função crescente se alguma das seguintes condições é satisfeita.

1. $\lambda_{21}>0, x_{2} \leq \frac{a+b}{2}$ e $P_{f}(b, a) \geq 0$;

2. $\lambda_{21}>0, x_{2}>\frac{a+b}{2}$ e $P_{f}(a, b) \geq 0$;

3. $\lambda_{21}<0, x_{1} \leq \frac{a+b}{2}$ e $P_{f}(b, b) \geq 0$;

4. $\lambda_{21}<0, x_{1}>\frac{a+b}{2}$ e $P_{f}(a, a) \geq 0$.

Prova: Ver a demonstração no Apêndice C.

Corolário 3.3.3. Seja $x_{1}=\frac{-\lambda_{11}}{2 \lambda_{21}}$ e $x_{2}=\frac{-\lambda_{11}+2 \lambda_{21}(a+b)}{2 \lambda_{21}}$. Então, $f_{X}$ é uma função decrescente se alguma das seguintes condições é satisfeita.

1. $\lambda_{21}>0, x_{1} \leq \frac{a+b}{2}$ e $P_{f}(b, b) \leq 0$;

2. $\lambda_{21}>0, x_{1}>\frac{a+b}{2}$ e $P_{f}(a, a) \leq 0$;

3. $\lambda_{21}<0, x_{2} \leq \frac{a+b}{2}$ e $P_{f}(b, a) \leq 0$;

4. $\lambda_{21}<0, x_{2}>\frac{a+b}{2}$ e $P_{f}(a, b) \leq 0$.

Prova: Ver a demonstração no Apêndice C.

Os dois corolários anteriores indicam possíveis coeficientes $\lambda_{10}, \lambda_{11}$ e $\lambda_{21}$ segundo a natureza crescente ou decrescente da marginal $f_{X}$. Salientamos que as relações anteriores não fornecem condições no coeficiente $\lambda_{01}$ do termo $y$. Na Tabela 3.3.1 mostramos valores dos coeficientes que foram obtidos aplicando os corolários anteriores.

Por exemplo, a primeira linha da Tabela 3.3 .1 foi construída utilizando o Corolário 3.3.2 (item 1). Isto significa que, quando $f_{X}$ for crescente em $[-1,1]$, os valores $\lambda_{10}=7, \lambda_{11}=4 \mathrm{e}$ $\lambda_{21}=1$ definem uma possível representação de $h$, já que satisfazem as condições

$$
\lambda_{21}>0, \quad x_{2} \leq \frac{a+b}{2} \quad \text { e } \quad P_{f}(b, a) \geq 0 .
$$




\section{Tabela 3.3 .1}

Possíveis valores dos coeficientes correspondentes a marginais $f_{X}$ crescentes ou decrescentes, para $(x, y) \in[-1,1] \times[-1,1]$ quando $h(x, y)=\exp \left(\lambda_{0}+\lambda_{10} x+\lambda_{01} y+\lambda_{11} x y+\lambda_{21} x^{2} y\right)$.

\begin{tabular}{|c||c|c|c|}
\hline$f_{X}$ & $\lambda_{10}$ & $\lambda_{11}$ & $\lambda_{21}$ \\
\hline \hline \multirow{3}{*}{$f_{X}^{i}$} & $\mathbf{7}$ & $\mathbf{4}$ & $\mathbf{1}$ \\
\cline { 2 - 4 } & 4 & -1 & 1 \\
\cline { 2 - 4 } & 7 & -4 & -1 \\
\hline \multirow{3}{*}{$f_{X}^{d}$} & -4 & 1 & 1 \\
\cline { 2 - 4 } & -4 & 1 & -1 \\
\cline { 2 - 4 } & -4 & -2 & -1 \\
\hline
\end{tabular}

A seguinte proposição fornece uma possível representação para o caso em que $f_{X}$ for uma função simétrica.

Proposição 3.3.4. Se $\lambda_{10}=0$ e $\frac{-\lambda_{11}}{2 \lambda_{21}}=\frac{a+b}{2}$, então a função $f_{X}$ é simétrica

Prova: Ver a demonstração no Apêndice C.

A seguir apresentamos relações entre os coeficientes para representarem $f_{X}$ como uma função com um ponto crítico.

Proposição 3.3.5. Se $\lambda_{10}=0$ e $\left.\frac{-\lambda_{11}}{2 \lambda_{21}} \in\right] a, b\left[\right.$, então $f_{X}$ possui um ponto crítico em $x_{0}=\frac{-\lambda_{11}}{2 \lambda_{21}}$. Se a $\geq 0, x_{0}$ é o único ponto crítico.

Prova: Ver a demonstração no Apêndice C.

Finalizamos a análise da marginal $f_{X}$ com os seguintes dois últimos resultados que dão informação sobre a sua unimodalidade e sobre a sua concavidade.

Proposição 3.3.6. Se $\left.a \geq 0, \lambda_{10}=0, \lambda_{21}<0 e \frac{-\lambda_{11}}{2 \lambda_{21}} \in\right] a, b\left[\right.$, então $f_{X}$ é unimodal com moda em $x_{0}=\frac{-\lambda_{11}}{2 \lambda_{21}}$.

Prova: Ver a demonstração no Apêndice C. 
Proposição 3.3.7. Se $\lambda_{21}>0$ e $a \geq 0$ ou se $\lambda_{21}<0$ e $b \leq 0$, então $f_{X}$ é convexa.

Prova: Ver a demonstração no Apêndice C.

\section{Propriedades de $g_{Y}$}

Seja $g_{Y}$ a densidade marginal da variável $Y$ dada pela equação (3.8). Na seguinte proposição mostramos relações entre os coeficientes segundo a $g_{Y}$ for crescente ou decrescente.

Proposição 3.3.8. Seja $P_{g}(x, 0)=\lambda_{01}+\lambda_{11} x+\lambda_{21} x^{2}$.

1. Se $\min _{x \in[a, b]} P_{g}(x, 0) \geq 0$, então $g_{Y}$ é uma função crescente;

2. Se $\max _{x \in[a, b]} P_{g}(x, 0) \leq 0$, então $g_{Y}$ é uma função decrescente.

Prova: A demonstração é análoga a prova da Proposição 3.2.21.

Os seguintes corolários apresentam os possíveis minimizadores e maximizadores de $P_{g}(\cdot, 0)$.

Corolário 3.3.9. A marginal $g_{Y}$ é uma função crescente, se alguma das seguintes condições é satisfeita.

1. $\lambda_{21}>0, a \leq-\frac{\lambda_{11}}{2 \lambda_{21}} \leq b$ e $P_{g}\left(-\frac{\lambda_{11}}{2 \lambda_{21}}, 0\right) \geq 0$;

2. $\lambda_{21}>0, a>-\frac{\lambda_{11}}{2 \lambda_{21}}$ e $P_{g}(a, 0) \geq 0$;

3. $\lambda_{21}>0, b<-\frac{\lambda_{11}}{2 \lambda_{21}}$ e $P_{g}(b, 0) \geq 0$;

4. $\lambda_{21}<0,-\frac{\lambda_{11}}{2 \lambda_{21}} \leq \frac{a+b}{2}$ e $P_{g}(b, 0) \geq 0$;

5. $\lambda_{21}<0,-\frac{\lambda_{11}}{2 \lambda_{21}}>\frac{a+b}{2}$ e $P_{g}(a, 0) \geq 0$.

Prova: Ver a demonstração no Apêndice C. 
Corolário 3.3.10. A marginal $g_{Y}$ é uma função decrescente, se alguma das seguintes condições é satisfeita.

1. $\lambda_{21}>0,-\frac{\lambda_{11}}{2 \lambda_{21}} \leq \frac{a+b}{2}$ e $P_{g}(b, 0) \leq 0$;

2. $\lambda_{21}>0,-\frac{\lambda_{11}}{2 \lambda_{21}}>\frac{a+b}{2}$ e $P_{g}(a, 0) \leq 0$;

3. $\lambda_{21}<0, a \leq-\frac{\lambda_{11}}{2 \lambda_{21}} \leq b$ e $P_{g}\left(-\frac{\lambda_{11}}{2 \lambda_{21}}, 0\right) \leq 0$;

4. $\lambda_{21}<0, a>-\frac{\lambda_{11}}{2 \lambda_{21}}$ e $P_{g}(a, 0) \leq 0$;

5. $\lambda_{21}<0, b<-\frac{\lambda_{11}}{2 \lambda_{21}}$ e $P_{g}(b, 0) \leq 0$.

Prova: Ver a demonstração no Apêndice C.

Finalizamos a análise da densidade marginal $g_{Y}$ com um resultado sobre a sua concavidade e um corolário de tal resultado.

Proposição 3.3.11. A densidade marginal $g_{Y}$ é uma função convexa.

Prova: Ver a demonstração no Apêndice C.

Corolário 3.3.12. Se $g_{Y}$ possuir um ponto mínimo, então $\left.r_{1} \in\right] a, b\left[\right.$ ou $\left.r_{2} \in\right] a, b[$, sendo

$$
r_{1}=\frac{-\lambda_{11}+\sqrt{\lambda_{11}^{2}-4 \lambda_{01} \lambda_{21}}}{2 \lambda_{21}} \quad \text { e } \quad r_{2}=\frac{-\lambda_{11}-\sqrt{\lambda_{11}^{2}-4 \lambda_{01} \lambda_{21}}}{2 \lambda_{21}} .
$$

Prova: A demonstração é conseqüência da convexidade de $g_{Y}$ e da Proposição 3.3.8. Observando que $r_{1}$ e $r_{2}$ são as raízes do polinômio $P_{g}(\cdot, 0)$ definido em tal proposição.

Realizadas as análises das duas marginais, podemos construir um exemplo. Na Figura 3.3.2 damos uma possível representação da densidade conjunta no caso de ser $f_{X}$ uma função com um ponto mínimo e uma moda e $g_{Y}$ uma função crescente.

Finalizamos observando que, segundo o nosso conhecimento, todos os resultados apresentados neste capítulo são originais. Em particular, o material das seções 3.1 e 3.2.1 foi apresentado no "Primer Congreso Latinoamericano de Estudiantes de Matemática" organizado pela Universidade de Chile e no $17^{\circ}$ SINAPE-Simpósio Nacional de Probabilidade e Estatística- organizado 
Figura 3.3.2

Densidade $h(x, y)=\exp \left(-1,529+y+x y-x^{2} y\right)$ para $(x, y) \in[-1,1] \times[-1,1]$.

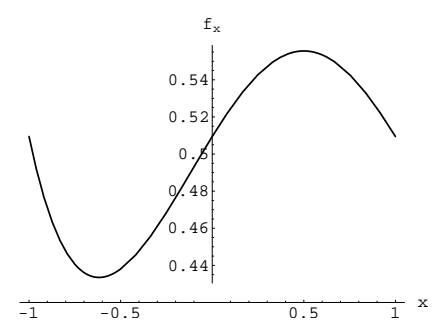

(a) Densidade marginal $f_{X}$.

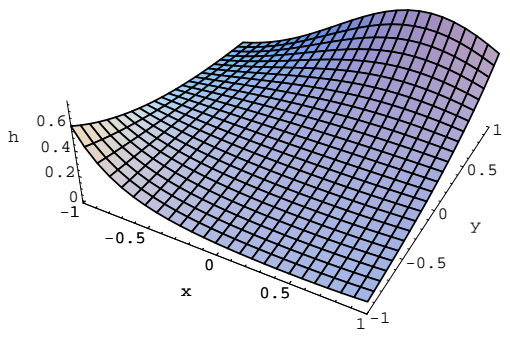

(c) Densidade conjunta $h$.

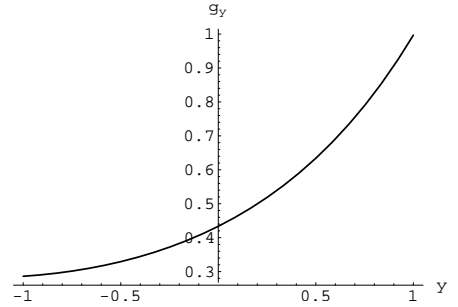

(b) Densidade marginal $g_{Y}$.

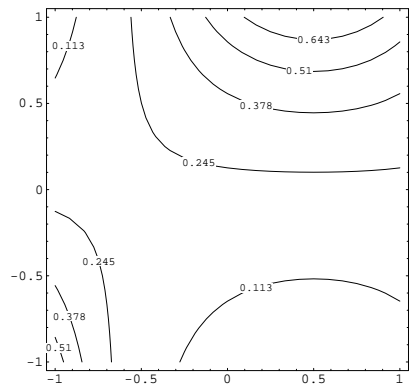

(d) Curvas de nível da densidade conjunta.

pela Associação Brasileira de Estatística. Enquanto que as seções 3.1, 3.2.1 e 3.3 foram expostas na "10 Escola Brasileira de Probabilidade", ver Fernández e Kolev (2006), também no "10th International Congress on Insurance: Mathematics and Economics", ver Kolev e Fernández (2006), e numa palestra no Institute of Applied Mathematics, Middle East Technical University, Ankara. O conteúdo completo do capítulo foi apresentado em um dos seminários de "Séries Temporais, Análise de Depêndencia e Aplicações em Atuaria e Finanças" realizado no Instituto de Matemática e Estatística da Universidade de São Paulo. Os eventos mencionados anteriormente foram realizados no ano de 2006. Concluímos salientando que com parte deste material, especificamente com as seções 3.1, 3.2.1 e 3.3, foi produzido um artigo de pesquisa que já foi aceito para publicação; ver Fernández e Kolev (2007b). 


\section{Capítulo 4}

\section{Comportamento geométrico das densidades condicionais}

A metodologia do capítulo anterior pode ser adaptada para o caso em que conhecemos o comportamento das densidades condicionais. Tal adaptação é apresentada no presente capítulo. Estudamos a influência do comportamento das densidades condicionais sobre os coeficientes da densidade conjunta. Os resultados apresentados servem para o caso em que é dada a natureza geométrica de ambas densidades condicionais ou de uma densidade condicional, por exemplo de $X$ dado $Y$, e da densidade marginal de $Y$. Este último caso combina os resultados do presente capítulo com os do anterior. No fim da análise de cada densidade fornecemos possíveis valores dos coeficientes segundo a forma da própria densidade conjunta.

\subsection{Polinômio de grau dois}

\subsubsection{Família de três parâmetros}

Consideramos

$$
h(x, y)=\exp \left(\lambda_{0}+\lambda_{10} x+\lambda_{01} y+\lambda_{11} x y\right), \quad(x, y) \in[a, b] \times[a, b]
$$


sendo $\lambda_{11} \neq 0$. Neste caso a densidade condicional de $X$ dado $Y$ é

$$
f_{X \mid Y}(x \mid y)= \begin{cases}\frac{\left(\lambda_{10}+\lambda_{11} y\right) \exp \left(\left(\lambda_{10}+\lambda_{11} y\right) x\right)}{\exp \left(\left(\lambda_{10}+\lambda_{11} y\right) b\right)-\exp \left(\left(\lambda_{10}+\lambda_{11} y\right) a\right)}, & \text { se } y \neq-\frac{\lambda_{10}}{\lambda_{11}} \\ \frac{1}{b-a}, & \text { se } y=-\frac{\lambda_{10}}{\lambda_{11}}\end{cases}
$$

já que

$$
g_{Y}(y)= \begin{cases}\exp \left(\lambda_{0}+\lambda_{01} y\right)\left(\frac{\exp \left(\left(\lambda_{10}+\lambda_{11} y\right) b\right)-\exp \left(\left(\lambda_{10}+\lambda_{11} y\right) a\right)}{\lambda_{10}+\lambda_{11} y}\right), & \text { se } y \neq-\frac{\lambda_{10}}{\lambda_{11}} \\ \exp \left(\lambda_{0}+\lambda_{01} y\right)(b-a), & \text { se } y=-\frac{\lambda_{10}}{\lambda_{11}} .\end{cases}
$$

Pela expressão da $f_{X \mid Y}$ é imediato obter a seguinte proposição.

Proposição 4.1.1. Seja $\lambda_{11} \in \mathbb{R}, \lambda_{11} \neq 0$.

1. Se $\lambda_{11}>0$, então

(a) $f_{X \mid Y}(\cdot \mid y)$ é uma função crescente para todo $y \in[a, b]$ se, e somente se, $\lambda_{10}+\lambda_{11} a>0$;

(b) $f_{X \mid Y}(\cdot \mid y)$ é uma função decrescente para todo $y \in[a, b]$ se, e somente se, $\lambda_{10}+\lambda_{11} b<0$

(c) $\lambda_{10}+\lambda_{11} a \leq 0 \leq \lambda_{10}+\lambda_{11} b$, então

i. $f_{X \mid Y}(\cdot \mid y)$ é uma função decrescente se, e somente se, $y<\frac{-\lambda_{10}}{\lambda_{11}}$;

ii. $f_{X \mid Y}(\cdot \mid y)$ é uma função constante se, e somente se, $y=\frac{-\lambda_{10}}{\lambda_{11}}$;

iii. $f_{X \mid Y}(\cdot \mid y)$ é uma função crescente se, e somente se, $y>\frac{-\lambda_{10}}{\lambda_{11}}$.

2. Se $\lambda_{11}<0$, então

(a) $f_{X \mid Y}(\cdot \mid y)$ é uma função crescente para todo $y \in[a, b]$ se, e somente se, $\lambda_{10}+\lambda_{11} b>0$;

(b) $f_{X \mid Y}(\cdot \mid y)$ é uma função decrescente para todo $y \in[a, b]$ se, e somente se, $\lambda_{10}+\lambda_{11} a<0$ 
(c) $\lambda_{10}+\lambda_{11} b \leq 0 \leq \lambda_{10}+\lambda_{11} a$, então
i. $f_{X \mid Y}(\cdot \mid y)$ é uma função decrescente se, e somente se, $y>\frac{-\lambda_{10}}{\lambda_{11}}$;
ii. $f_{X \mid Y}(\cdot \mid y)$ é uma função constante se, e somente se, $y=\frac{-\lambda_{10}}{\lambda_{11}}$;
iii. $f_{X \mid Y}(\cdot \mid y)$ é uma função crescente se, e somente se, $y<\frac{-\lambda_{10}}{\lambda_{11}}$.

Prova: Conseqüência imediata da expressão de $f_{X \mid Y}$ dada na equação (4.1).

$\mathrm{Na}$ proposição a seguir enunciamos a convexidade de $f_{X \mid Y}$.

Proposição 4.1.2. A densidade condicional $f_{X \mid Y}(\cdot \mid y)$ é uma função convexa para todo $y \in[a, b]$. Se $f_{X \mid Y}(\cdot \mid y)$ não é constante, então é estritamente convexa.

Prova: Segue do fato que, pela equação $(4.1), f_{X \mid Y}(\cdot \mid y)$ é uma função exponencial ou uma função constante.

As propriedades anteriores, junto com os resultados da seção 3.2.1, nos permitem estudar a influência sobre a densidade conjunta do comportamento de $g_{Y}$ e de $f_{X \mid Y}$. Na Tabela 4.1.1 damos um resumo.

Pode ser visto na Tabela 4.1.1 que quando $g_{Y}$ é crescente e $f_{X \mid Y}(\cdot \mid y)$ é decrescente para todo $y \in[a, b]$, os coeficientes $\lambda_{10}, \lambda_{01}$ e $\lambda_{11}$ devem satisfazer as seguintes relações

$$
\begin{aligned}
& L\left(a, \lambda_{10} ; \lambda_{11}\right) \leq \lambda_{01}, \quad \lambda_{11}>0 \quad \text { e } \quad \lambda_{10}+\lambda_{11} b<0 \quad \text { ou } \\
& L\left(a, \lambda_{10} ; \lambda_{11}\right) \leq \lambda_{01}, \quad \lambda_{11}<0 \quad \text { e } \quad \lambda_{10}+\lambda_{11} a<0 .
\end{aligned}
$$




\section{Tabela 4.1.1}

Condições encontradas para os coeficientes $\lambda_{10}, \lambda_{01}$ e $\lambda_{11}$ dadas as diferentes densidades marginais e condicionais para $h(x, y)=\exp \left(\lambda_{0}+\lambda_{10} x+\lambda_{01} y+\lambda_{11} x y\right)$ e $\left.y_{0} \in\right] a, b[$.

\begin{tabular}{|c|c|c|}
\hline & $f_{X \mid Y}^{i}(\cdot \mid y)$ para todo $y \in[a, b]$ & $f_{X \mid Y}^{d}(\cdot \mid y)$ para todo $y \in[a, b]$ \\
\hline$g_{Y}^{i}$ & $\begin{array}{c}L\left(a, \lambda_{10} ; \lambda_{11}\right) \leq \lambda_{01} \\
\mathrm{e} \\
{\left[\left(\lambda_{11}>0 \text { e } \lambda_{10}+\lambda_{11} a>0\right)\right.} \\
\text { ou } \\
\left.\left(\lambda_{11}<0 \text { e } \lambda_{10}+\lambda_{11} b>0\right)\right]\end{array}$ & $\begin{array}{c}L\left(a, \lambda_{10} ; \lambda_{11}\right) \leq \lambda_{01} \\
\text { e } \\
{\left[\left(\lambda_{11}>0 \text { e } \lambda_{10}+\lambda_{11} b<0\right)\right.} \\
\text { ou } \\
\left.\left(\lambda_{11}<0 \text { e } \lambda_{10}+\lambda_{11} a<0\right)\right]\end{array}$ \\
\hline$g_{Y}^{d}$ & $\begin{array}{c}L\left(b, \lambda_{10} ; \lambda_{11}\right) \geq \lambda_{01} \\
\mathrm{e} \\
{\left[\left(\lambda_{11}>0 \text { e } \lambda_{10}+\lambda_{11} a>0\right)\right.} \\
\text { ou } \\
\left.\left(\lambda_{11}<0 \text { e } \lambda_{10}+\lambda_{11} b>0\right)\right]\end{array}$ & $\begin{array}{c}L\left(b, \lambda_{10} ; \lambda_{11}\right) \geq \lambda_{01} \\
\mathrm{e} \\
{\left[\left(\lambda_{11}>0 \text { e } \lambda_{10}+\lambda_{11} b<0\right)\right.} \\
\text { ou } \\
\left(\lambda_{11}<0 \text { e } \lambda_{10}+\lambda_{11} a<0\right)\end{array}$ \\
\hline$g_{Y}^{m}$ & $\begin{array}{c}L\left(y_{0}, \lambda_{10} ; \lambda_{11}\right)=\lambda_{01} \\
\mathrm{e} \\
{\left[\left(\lambda_{11}>0 \text { and } \lambda_{10}+\lambda_{11} a>0\right)\right.} \\
\text { ou } \\
\left.\left(\lambda_{11}<0 \text { e } \lambda_{10}+\lambda_{11} b>0\right)\right]\end{array}$ & $\begin{array}{c}L\left(y_{0}, \lambda_{10} ; \lambda_{11}\right)=\lambda_{01} \\
\mathrm{e} \\
{\left[\left(\lambda_{11}>0 \text { e } \lambda_{10}+\lambda_{11} b<0\right)\right.} \\
\text { ou } \\
\left(\lambda_{11}<0 \text { e } \lambda_{10}+\lambda_{11} a<0\right)\end{array}$ \\
\hline
\end{tabular}

A título de exemplo, na Figura 4.1.2 apresentamos uma possível representação de uma densidade conjunta com domínio $[0,2] \times[0,2]$, sendo $g_{Y}$ decrescente, $f_{X \mid Y}(\cdot \mid y)$ decrescente para $y \in\left[0,1\left[, f_{X \mid Y}(\cdot \mid y=1)\right.\right.$ constante e $f_{X \mid Y}(\cdot \mid y)$ crescente para $\left.\left.y \in\right] 1,2\right]$. 
Figura 4.1.2

Densidade $h(x, y)=\exp (0,015-x-1,5 y+x y)$ para $(x, y) \in[0,2] \times[0,2]$.

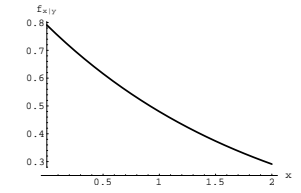

(a) $f_{X \mid Y}(x \mid y=0,5)$

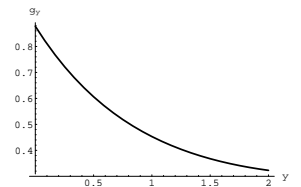

(d) Densidade $g_{Y}$.

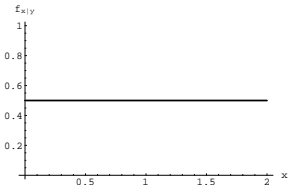

(b) $f_{X \mid Y}(x \mid y=1)$

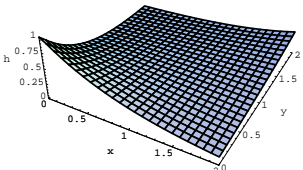

(e) Densidade conjunta $h$.

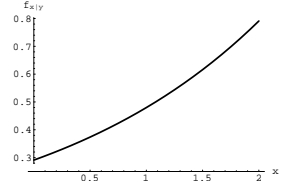

(c) $f_{X \mid Y}(x \mid y=1,5)$

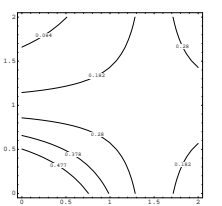

(f) Curvas de nível da densidade conjunta.

É interessante ver como os resultados obtidos na análise das densidades condicionais podem ser aplicados numa nova abordagem do problema. Podemos assumir que conhecemos o comportamento geométrico da densidade conjunta e assim determinar os valores possíveis dos seus coeficientes. Mais especificamente, assumimos que conhecemos a forma das funções definidas pelas interseções de planos do tipo $x=$ cte e $y=$ cte com o gráfico da densidade conjunta $h$ dado por

$$
G_{h}=\left\{(x, y, z) \in \mathbb{R}^{3}:(x, y) \in[a, b] \times[a, b] \text { e } z=h(x, y)\right\} .
$$

Seja $k \in[a, b]$. Estudar a interseção de $G_{h}$ com o plano $y=k$ é equivalente a estudar o comportamento de $f_{X \mid Y}(x \mid y=k)$, pois

$$
G_{h} \cap\left\{(x, y, z) \in \mathbb{R}^{3}: x \in[a, b] \text { e } y=k\right\}=\{(x, k, h(x, k)) \in[a, b] \times\{k\} \times] 0,+\infty[\}
$$

e, por outro lado,

$$
h(x, k)=f_{X \mid Y}(x \mid y=k) g_{Y}(k) .
$$


Como $g_{Y}(k)$ é um número positivo, então os possíveis comportamentos de $h(x, k)$ e de $f_{X \mid Y}(x \mid y=k)$ são iguais para todo $x \in[a, b]$. Similarmente, temos a equivalência da natureza geométrica entre $h(k, y)$ e $g_{Y \mid X}(y \mid x=k)$. Assim obtemos a seguinte proposição, lembrando que sgn é a função sinal definida na equação (3.7).

Proposição 4.1.3. Seja $\lambda_{11} \in \mathbb{R}, \lambda_{11} \neq 0$ e $k \in[a, b]$.

1. $h(\cdot, k)$ é decrescente se, e somente se, $\operatorname{sgn}\left(\lambda_{11}\right) k<-\frac{\lambda_{10}}{\left|\lambda_{11}\right|}$;

2. $h(\cdot, k)$ é constante se, e somente se, $k=-\frac{\lambda_{10}}{\lambda_{11}}$;

3. $h(\cdot, k)$ é crescente se, e somente se, $\operatorname{sgn}\left(\lambda_{11}\right) k>-\frac{\lambda_{10}}{\left|\lambda_{11}\right|}$;

4. $h(k, \cdot)$ é decrescente se, e somente se, $\operatorname{sgn}\left(\lambda_{11}\right) k<-\frac{\lambda_{01}}{\left|\lambda_{11}\right|}$;

5. $h(k, \cdot)$ é constante se, e somente se, $k=-\frac{\lambda_{01}}{\lambda_{11}}$;

6. $h(k, \cdot)$ é crescente se, e somente se, $\operatorname{sgn}\left(\lambda_{11}\right) k>-\frac{\lambda_{01}}{\left|\lambda_{11}\right|}$.

Prova: Conseqüência da equação (4.2) e da Proposição 4.1.1.

Proposição 4.1.4. Seja $k \in[a, b]$. As funções $h(\cdot, k)$ e $h(k, \cdot)$ são convexas.

Prova: Segue da equação (4.2) e da Proposição 4.1.2.

Com o exposto até aqui, para $\lambda_{11}$ fixo, podemos determinar no plano $\left(\lambda_{10}, \lambda_{01}\right)$ as regiões dos diferentes comportamentos da densidade conjunta. Em particular, consideramos $\lambda_{11}>0$ e combinamos, na Figura 4.1.3, os comportamentos das marginais (apresentados na Figura 3.2.4) com os da densidade conjunta. Na Figura 4.1 .3 observamos, por exemplo, que se $h(\cdot, k)$ e $h(k, \cdot)$ são crescentes para todo $k \in[a, b]$, então $\lambda_{10} \geq-a \lambda_{11}$ e $\lambda_{01} \geq-a \lambda_{11}$ e portanto ambas marginais são crescentes (ver Proposição 3.2.4). Para $\lambda_{11}<0$ obtemos um gráfico similar.

A seguinte proposição apresenta dois casos para os quais podemos afirmar que a densidade $h$ possui um ponto de sela.

Proposição 4.1.5. Se $f_{X}$ e $g_{Y}$ possuem um ponto mínimo ou se $f_{X \mid Y}$ e $g_{Y \mid X}$ são constantes para algum $x$ e $y \in] a, b[$, então h possui um ponto de sela.

Prova: Ver a demonstração no Apêndice D. 
Figura 4.1.3

Regiões do plano $\left(\lambda_{10}, \lambda_{01}\right)$ correspondentes aos diferentes tipos de densidades marginais e conjunta para $\lambda_{11}>0$ fixo quando $h(x, y)=\exp \left(\lambda_{0}+\lambda_{10} x+\lambda_{01} y+\lambda_{11} x y\right)$.

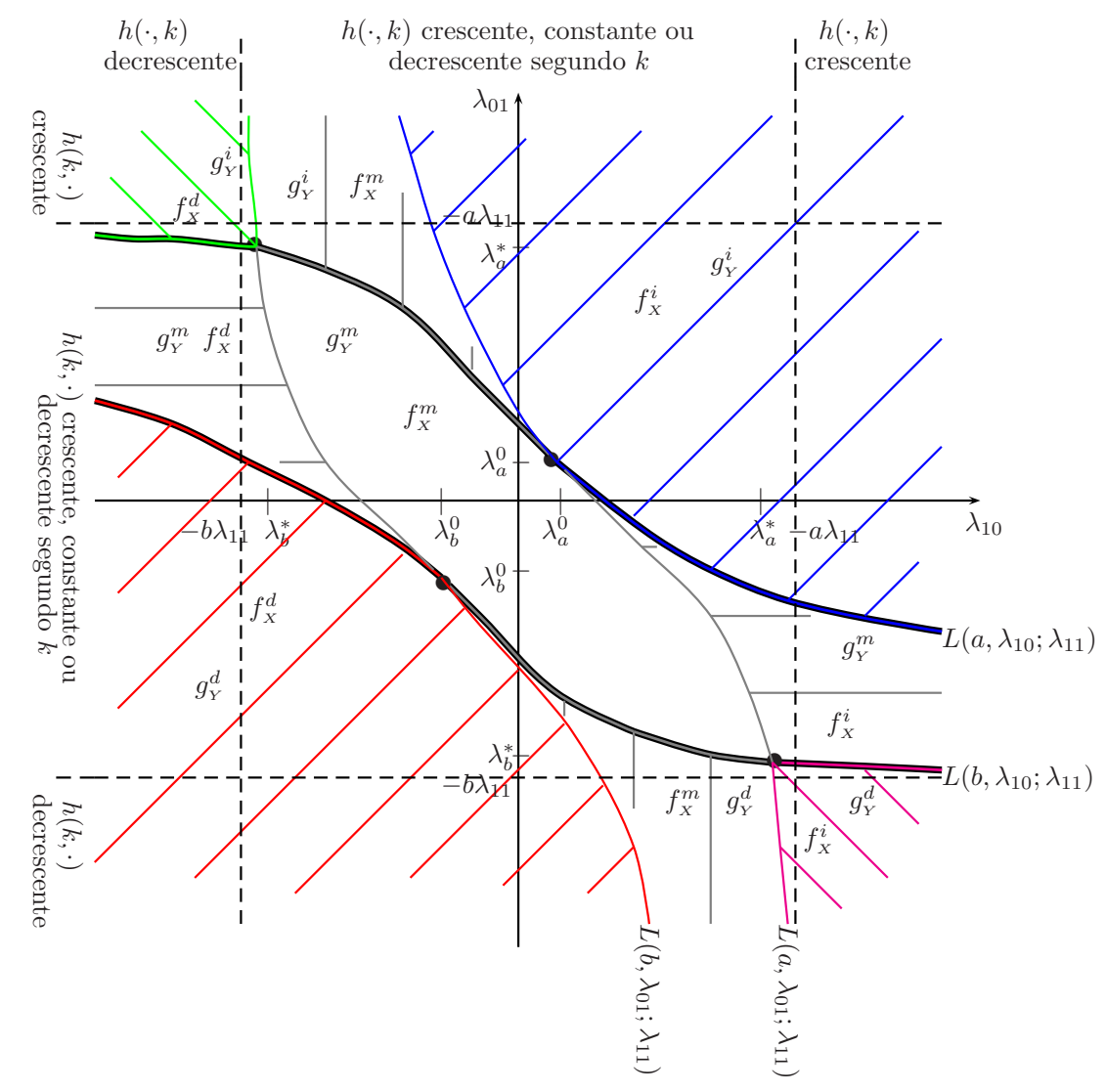

Corolário 4.1.6. A densidade h possui no máximo um ponto crítico. Tal ponto é ponto de sela de h e está dado por

$$
\left(x_{0}, y_{0}\right)=\left(-\frac{\lambda_{01}}{\lambda_{11}},-\frac{\lambda_{10}}{\lambda_{11}}\right) .
$$

Prova: Conseqüência imediata do gradiente e a matriz hessiana de $h$ calculados na demonstração da Proposição 4.1.5. 
Concluímos a presente seção observando que a densidade $h$ dada na Figura 4.1.2 possui o seguinte comportamento (segundo a Proposição 4.1.3). $h(\cdot, k)$ é decrescente para $k<1$, constante para $k=1$ e crescente para $k>1$. Enquanto $h(k, \cdot)$ é decrescente para $k<1,5$, constante para $k=1,5$ e crescente para $k>1,5$.

\subsubsection{Família de quatro parâmetros}

A seguir consideramos

$$
h(x, y)=\exp \left(\lambda_{0}+\lambda_{10} x+\lambda_{01} y+\lambda_{11} x y+\lambda_{20} x^{2}\right), \quad(x, y) \in[a, b] \times[a, b]
$$

sendo $\lambda_{11} \neq 0$ e $\lambda_{20} \neq 0$. Assumimos $\lambda_{11} \neq 0$ para ter dependência entre as variáveis.

Quando a densidade conjunta $h$ é dada por (4.3), a correspondente densidade condicional de $X$ dado $Y$ resulta ser

$$
f_{X \mid Y}(x \mid y)=\frac{\exp \left(\lambda_{10} x+\lambda_{11} x y+\lambda_{20} x^{2}\right)}{\int_{a}^{b} \exp \left(\left(\lambda_{10}+\lambda_{11} y\right) u+\lambda_{20} u^{2}\right) d u}
$$

pois

$$
g_{Y}(y)=\exp \left(\lambda_{0}+\lambda_{01} y\right) \int_{a}^{b} \exp \left(\left(\lambda_{10}+\lambda_{11} y\right) x+\lambda_{20} x^{2}\right) d x .
$$

Por outro lado, a densidade de $Y$ dado $X$ é dada por

$$
g_{Y \mid X}(y \mid x)= \begin{cases}\frac{\left(\lambda_{01}+\lambda_{11} x\right) \exp \left(\left(\lambda_{01}+\lambda_{11} x\right) y\right)}{\exp \left(\left(\lambda_{01}+\lambda_{11} x\right) b\right)-\exp \left(\left(\lambda_{01}+\lambda_{11} x\right) a\right)}, & \text { se } \lambda_{01}+\lambda_{11} x \neq 0 \\ \frac{1}{b-a}, & \text { se } \lambda_{01}+\lambda_{11} x=0\end{cases}
$$

já que

$$
f_{X}(x)= \begin{cases}\exp \left(\lambda_{0}+\lambda_{10} x+\lambda_{20} x^{2}\right)\left(\frac{\exp \left(\left(\lambda_{01}+\lambda_{11} x\right) b\right)-\exp \left(\left(\lambda_{01}+\lambda_{11} x\right) a\right)}{\lambda_{01}+\lambda_{11} x}\right), & \text { se } \lambda_{01}+\lambda_{11} x \neq 0 \\ \exp \left(\lambda_{0}+\lambda_{10} x+\lambda_{20} x^{2}\right)(b-a), & \text { se } \lambda_{01}+\lambda_{11} x=0 .\end{cases}
$$

Para a densidade que estamos considerando aqui, dada pela equação (4.3), é evidente que o estudo de $g_{Y \mid X}$ e de $f_{X \mid Y}$ é diferente. A análise da densidade condicional $g_{Y \mid X}$ é análoga ao realizado para as densidades condicionais na seção anterior, seção 4.1.1. Ou seja, $g_{Y \mid X}(\cdot \mid x)$ é 
uma função convexa para todo $x \in[a, b]$ e é válida a Proposição 4.1.1 substituindo $f_{X \mid Y}$ por $g_{Y \mid X}, y$ por $x$ e $\lambda_{10}$ por $\lambda_{01}$.

A seguir analisamos o caso em que assumimos conhecido o comportamento geométrico de $f_{X \mid Y}$. Começamos com o seguinte lema sobre a unicidade de um possível ponto crítico de $f_{X \mid Y}$.

Lema 4.1.7. Para cada $y \in[a, b], f_{X \mid Y}(\cdot \mid y)$ pode ter no máximo um ponto crítico.

Prova: Ver a demonstração no Apêndice D.

Quando a densidade não possui ponto crítico, ou seja, é crescente ou decescente, obtemos as seguintes relações entre os coeficientes.

Proposição 4.1.8. Para cada $y \in[a, b]$ obtemos que

1. Se $\lambda_{20}>0$ e $\lambda_{10}+\lambda_{11} y+2 \lambda_{20} a \geq 0$ ou se $\lambda_{20}<0$ e $\lambda_{10}+\lambda_{11} y+2 \lambda_{20} b \geq 0$, então $f_{X \mid Y}(\cdot \mid y)$ é crescente;

2. Se $\lambda_{20}>0$ e $\lambda_{10}+\lambda_{11} y+2 \lambda_{20} b \leq 0$ ou se $\lambda_{20}<0$ e $\lambda_{10}+\lambda_{11} y+2 \lambda_{20} a \leq 0$, então $f_{X \mid Y}(\cdot \mid y)$ é decrescente.

Prova: Ver a demonstração no Apêndice D.

Com o objetivo de construir uma tabela com as relações entre os coeficientes segundo o comportamento de $f_{X \mid Y}(\cdot \mid y)$, definimos os seguintes pontos

$$
\begin{aligned}
& y_{a} \stackrel{\text { def }}{=} \frac{-\lambda_{10}-2 \lambda_{20} a}{\lambda_{11}}, \\
& y_{b} \stackrel{\text { def }}{=} \frac{-\lambda_{10}-2 \lambda_{20} b}{\lambda_{11}} .
\end{aligned}
$$

É imediato ver que $\lambda_{11} \lambda_{20}<0$ implica que $y_{a}<y_{b}$ e $\lambda_{11} \lambda_{20}>0$ implica que $y_{b}<y_{a}$. O seguinte lema mostra que para quaisquer valores de $y_{a}$ e $y_{b}$ existem coeficientes que satisfazem as igualdades acima. 
Lema 4.1.9. Para quaisquer $c$ e $d \in \mathbb{R}, c \neq d$, existem números reais $\lambda_{10}, \lambda_{11}$ e $\lambda_{20}$ tais que $y_{a}=c$ e $y_{b}=d$.

Prova: Ver a demonstração no Apêndice D.

Na Tabela 4.1 .4 damos os valores de $y$ para os quais $f_{X \mid Y}(\cdot \mid y)$ é crescente, assim como os valores para o caso decrescente. Por exemplo, vemos que se $\lambda_{20}>0, \lambda_{11}>0$ e $y \geq y_{a}$, ou seja $2 \lambda_{20} a+\lambda_{11} y \geq-\lambda_{10}$, então $f_{X \mid Y}(\cdot \mid y)$ é crescente.

\section{Tabela 4.1.4}

Valores de $y$ para $f_{X \mid Y}(\cdot \mid y)$ crescente (tabela a esquerda) e $f_{X \mid Y}(\cdot \mid y)$ decrescente (tabela a direita).

\begin{tabular}{|c|c|c|}
\hline$\lambda_{20}$ & $\lambda_{11}$ & $y \in[a, b] \cap A$ \\
\hline \hline+ & + & $\boldsymbol{A}=\left[\boldsymbol{y}_{\boldsymbol{a}},+\infty[\right.$ \\
\hline+ & - & $\left.A=]-\infty, y_{a}\right]$ \\
\hline- & + & $A=\left[y_{b},+\infty[\right.$ \\
\hline- & - & $\left.A=]-\infty, y_{b}\right]$ \\
\hline
\end{tabular}

\begin{tabular}{|c|c|c|}
\hline$\lambda_{20}$ & $\lambda_{11}$ & $y \in[a, b] \cap A$ \\
\hline \hline+ & + & $\left.A=]-\infty, y_{b}\right]$ \\
\hline+ & - & $A=\left[y_{b},+\infty[\right.$ \\
\hline- & + & $\left.A=]-\infty, y_{a}\right]$ \\
\hline- & - & $A=\left[y_{a},+\infty[\right.$ \\
\hline
\end{tabular}

Para completar o estudo das regiões dos diferentes comportamentos de $f_{X \mid Y}$ precisamos estudar a sua segunda derivada, ou seja, a sua concavidade. A segunda derivada está dada por

$$
\frac{\partial^{2} f_{X \mid Y}}{\partial x^{2}}(x \mid y)=f_{X \mid Y}(x \mid y)\left(2 \lambda_{20}+\left(\lambda_{10}+\lambda_{11} y+2 \lambda_{20} x\right)^{2}\right)
$$

É imediato ver que quando $\lambda_{20}>0, f_{X \mid Y}(\cdot \mid y)$ é uma função estritamente convexa para todo $y \in[a, b]$. Por outro lado, quando $\lambda_{20}<0$ obtemos que a segunda derivada no ponto crítico $x_{0}=\frac{-\lambda_{10}-\lambda_{11} y}{2 \lambda_{20}}$ é negativa, já que $\left.\frac{\partial^{2} f_{X \mid Y}}{\partial x^{2}}(x \mid y)\right|_{x=x_{0}}=2 \lambda_{20} f_{X \mid Y}\left(x_{0} \mid y\right)$. Em particular, concluímos que $f_{X \mid Y}$ não possui ponto de inflexão, ou seja, $f_{X \mid Y}(\cdot \mid y)$ é uma função convexa ou côncava. Pelo Lema 4.1.7, a Tabela 4.1.4 e as observações anteriores, obtemos a Tabela 4.1 .5 com a caracterização dos casos em que $f_{X \mid Y}$ possui ponto crítico. 


\section{Tabela 4.1.5}

Valores de $y$ para quando $f_{X \mid Y}(\cdot \mid y)$ possui um ponto mínimo (tabela a esquerda) e um ponto máximo (tabela a direita).

\begin{tabular}{|c|c|c|}
\hline$\lambda_{20}$ & $\lambda_{11}$ & $y \in[a, b] \cap A$ \\
\hline \hline+ & + & $A=] y_{b}, y_{a}[$ \\
\hline+ & - & $A=] y_{a}, y_{b}[$ \\
\hline
\end{tabular}

\begin{tabular}{|c|c|c|}
\hline$\lambda_{20}$ & $\lambda_{11}$ & $y \in[a, b] \cap A$ \\
\hline \hline- & + & $A=] y_{a}, y_{b}[$ \\
\hline- & - & $\boldsymbol{A}=] \boldsymbol{y}_{\boldsymbol{b}}, \boldsymbol{y}_{\boldsymbol{a}}[$ \\
\hline
\end{tabular}

Em particular, a informação da Tabela 4.1 .5 nos permite inferir que se $\lambda_{20}<0, \lambda_{11}<0$ e $y \in] y_{b}, y_{a}\left[\right.$, isto é $\lambda_{11} y+2 \lambda_{20} b<-\lambda_{10}<\lambda_{11} y+2 \lambda_{20} a$, então $f_{X \mid Y}(\cdot \mid y)$ é unimodal.

A seguir estudamos a concavidade de $f_{X \mid Y}(\cdot \mid y)$ para $\lambda_{20}<0$ quando $f_{X \mid Y}(\cdot \mid y)$ não possui ponto crítico, ou seja, $f_{X \mid Y}(\cdot \mid y)$ é crescente ou decrescente. Este caso é apresentado na seguinte proposição.

Proposição 4.1.10. Seja $\lambda_{20}<0$.

1. Se $f_{X \mid Y}(\cdot \mid y)$ é crescente, então $-2 \lambda_{20} \leq\left(\lambda_{10}+\lambda_{11} y+2 \lambda_{20} b\right)^{2}$ se, e somente se, $f_{X \mid Y}(\cdot \mid y)$ é convexa;

2. Se $f_{X \mid Y}(\cdot \mid y)$ é crescente, então $-2 \lambda_{20} \geq\left(\lambda_{10}+\lambda_{11} y+2 \lambda_{20} a\right)^{2}$ se, e somente se, $f_{X \mid Y}(\cdot \mid y)$ é côncava;

3. Se $f_{X \mid Y}(\cdot \mid y)$ é decrescente, então $-2 \lambda_{20} \leq\left(\lambda_{10}+\lambda_{11} y+2 \lambda_{20} a\right)^{2}$ se, e somente se, $f_{X \mid Y}(\cdot \mid y)$ é convexa;

4. Se $f_{X \mid Y}(\cdot \mid y)$ é decrescente, então $-2 \lambda_{20} \geq\left(\lambda_{10}+\lambda_{11} y+2 \lambda_{20} b\right)^{2}$ se, e somente se, $f_{X \mid Y}(\cdot \mid y)$ é côncava.

Prova: Ver a demonstração no Apêndice D.

O seguinte corolário mostra que a concavidade de $f_{X \mid Y}(\cdot \mid y)$ é a mesma para qualquer $y \in[a, b]$. 
Corolário 4.1.11. Seja $y_{0} \in[a, b]$.

1. Se $f_{X \mid Y}\left(\cdot \mid y=y_{0}\right)$ é convexa, então $f_{X \mid Y}(\cdot \mid y)$ é convexa para todo $y \in[a, b]$;

2. Se $f_{X \mid Y}\left(\cdot \mid y=y_{0}\right)$ é côncava, então $f_{X \mid Y}(\cdot \mid y)$ é côncava para todo $y \in[a, b]$.

Prova: Ver a demonstração no Apêndice D.

A título de exemplificação de como utilizar os resultados apresentados, fornecemos a construção de uma densidade conjunta com as seguintes características. O domínio é dado por $[-3,10] \times[-3,10], f_{X \mid Y}(\cdot \mid y)$ é decrescente para $-3 \leq y \leq 0$, unimodal para $0<y<5$ e crescente para $5 \leq y \leq 10$. Pela Tabela $4.1 .5, \lambda_{20}$ deve ser negativo. Usando esta informação e a Tabela 4.1.4, concluímos que $\lambda_{11}>0$. Consequentemente $y_{a}<y_{b}$, isto junto com a informação dada de $f_{X \mid Y}$, nos permite concluir que $y_{a}=0$ e $y_{b}=5$, então

$$
\left\{\begin{array}{l}
0=\frac{-\lambda_{10}+6 \lambda_{20}}{\lambda_{11}} \\
5=\frac{-\lambda_{10}+20 \lambda_{20}}{\lambda_{11}}
\end{array}\right.
$$

Escolhemos os coeficientes $\lambda_{10}, \lambda_{11}$ e $\lambda_{20}$ satisfazendo as relações acima, a existência esta garantida pelo Lema 4.1.9. O coeficiente $\lambda_{01}$ depende da natureza geométrica de $g_{Y}$ ou de $g_{Y \mid X}$.

Assim como na seção anterior, podemos inferir relações entre os coeficientes segundo o comportamento geométrico da própria densidade conjunta. Tais relações são equivalentes às já apresentadas para as densidades condicionais. Pela equação (4.2) vemos que para cada $k \in[a, b]$ a função $h(\cdot, k)$ possui o mesmo comportamento de $f_{X \mid Y}(\cdot \mid y=k)$ e $h(k, \cdot)$ o mesmo de $g_{Y \mid X}(\cdot \mid x=k)$. Finalizamos com a seguinte proposição sobre a existência de pontos críticos da densidade conjunta.

Proposição 4.1.12. A densidade h possui no máximo um ponto crítico. Tal ponto é ponto de sela de $h$ e é dado por

$$
\left(x_{0}, y_{0}\right)=\left(-\frac{\lambda_{01}}{\lambda_{11}}, \frac{2 \lambda_{20} \lambda_{01}}{\lambda_{11}^{2}}-\frac{\lambda_{10}}{\lambda_{11}}\right) \text {. }
$$

Prova: Ver a demonstração no Apêndice D. 


\subsubsection{Família de cinco parâmetros}

Consideramos

$$
h(x, y)=\exp \left(\lambda_{0}+\lambda_{10} x+\lambda_{01} y+\lambda_{11} x y+\lambda_{20} x^{2}+\lambda_{02} y^{2}\right), \quad(x, y) \in[a, b] \times[a, b]
$$

sendo $\lambda_{11} \neq 0, \lambda_{20} \neq 0$ e $\lambda_{02} \neq 0$. Assumimos $\lambda_{11} \neq 0$ para ter dependência.

Quando a densidade conjunta $h$ é dada por (4.7), a correspondente densidade condicional de $X$ dado $Y$ é

$$
f_{X \mid Y}(x \mid y)=\frac{\exp \left(\lambda_{10} x+\lambda_{11} x y+\lambda_{20} x^{2}\right)}{\int_{a}^{b} \exp \left(\left(\lambda_{10}+\lambda_{11} y\right) u+\lambda_{20} u^{2}\right) d u}
$$

já que

$$
g_{Y}(y)=\exp \left(\lambda_{0}+\lambda_{01} y+\lambda_{02} y^{2}\right) \int_{a}^{b} \exp \left(\left(\lambda_{10}+\lambda_{11} y\right) x+\lambda_{20} x^{2}\right) d x .
$$

Neste caso, assim como na seção 4.1.1, basta estudar uma das densidades condicionais já que existe simetria entre os coeficientes. Os resultados de uma são facilmente adaptados à outra densidade, pois ambas possuem a mesma forma, mas com coeficientes diferentes.

A expressão de $f_{X \mid Y}$ coincide com a densidade condicional de $X$ dado $Y$ para quando $h$ é aproximada por (4.3), caso estudado na seção 4.1.2. Logo, os resultados apresentados em tal seção são válidos para o presente caso. Portanto passamos diretamente ao estudo do comportamento da densidade conjunta, em particular dos seus possíveis pontos críticos.

Proposição 4.1.13. A densidade h possui no máximo um ponto crítico dado por

$$
\left(x_{0}, y_{0}\right)=\left(\frac{-\lambda_{01} \lambda_{11}+2 \lambda_{02} \lambda_{10}}{\lambda_{11}^{2}-4 \lambda_{02} \lambda_{20}},-\frac{\lambda_{10}}{\lambda_{11}}-2 \frac{\lambda_{20}}{\lambda_{11}}\left(\frac{-\lambda_{01} \lambda_{11}+2 \lambda_{02} \lambda_{10}}{\lambda_{11}^{2}-4 \lambda_{02} \lambda_{20}}\right)\right) .
$$

Prova: Ver a demonstração no Apêndice D.

A seguir analisamos com detalhe o caso da existência de ponto crítico, ou seja, assumimos que $\lambda_{11}^{2}-4 \lambda_{02} \lambda_{20} \neq 0$ e que $\left.\left(x_{0}, y_{0}\right) \in\right] a, b[\times] a, b\left[\right.$ para $\left(x_{0}, y_{0}\right)$ definido na equação (4.8). Na Proposição 4.1.14 mostramos as relações entre os coeficientes $\lambda_{11}, \lambda_{02}$ e $\lambda_{20}$ segundo o ponto crítico for um maximizador, um minimizador ou um ponto de sela de $h$. 
Proposição 4.1.14. Se $h$ possui um ponto crítico $\left.\left(x_{0}, y_{0}\right) \in\right] a, b[\times] a, b[$, então

1. $\lambda_{20}+\lambda_{02}=0$ implica que $\left(x_{0}, y_{0}\right)$ é ponto de sela;

2. $\lambda_{11}^{2}-4 \lambda_{02} \lambda_{20}<0<\lambda_{20}+\lambda_{02}$ implica que $\left(x_{0}, y_{0}\right)$ é um minimizador;

3. $\lambda_{11}^{2}-4 \lambda_{02} \lambda_{20}<0$ e $0>\lambda_{20}+\lambda_{02}$ implicam que $\left(x_{0}, y_{0}\right)$ é um maximizador;

4. $\lambda_{11}^{2}-4 \lambda_{02} \lambda_{20}>0$ implica que $\left(x_{0}, y_{0}\right)$ é ponto de sela.

Prova: Ver a demonstração no Apêndice D.

Finalizamos o estudo do presente caso com a Figura 4.1.6, na qual apresentamos uma densidade conjunta e as correspondentes curvas de nível. A densidade mostrada possui um maximizador ou moda (os coeficientes foram escolhidos segundo as Proposições 4.1.13 e 4.1.14).

Figura 4.1.6

Densidade $h(x, y)=\exp \left(-1,858-2 x+x y-x^{2}-2 y^{2}\right)$, para $(x, y) \in[-2,1] \times[-2,1]$.

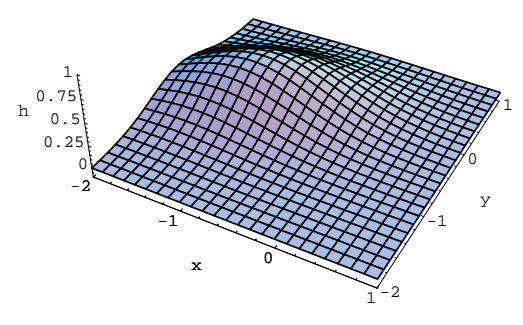

(a) Densidade conjunta $h$.

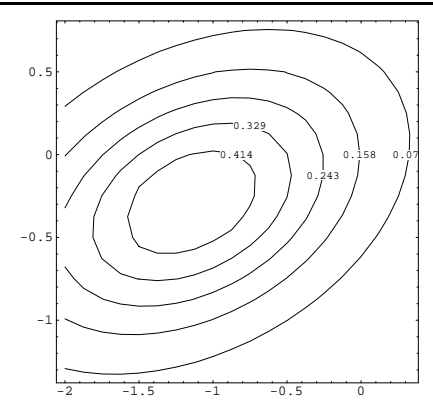

(b) Curvas de nível da densidade conjunta. 


\subsection{Polinômio de grau três}

Finalmente assumimos que

$$
h(x, y)=\exp \left(\lambda_{0}+\lambda_{10} x+\lambda_{01} y+\lambda_{11} x y+\lambda_{21} x^{2} y\right), \quad(x, y) \in[a, b] \times[a, b]
$$

sendo $\lambda_{21} \neq 0$.

Quando a densidade conjunta $h$ é dada por (4.9), a correspondente densidade condicional de $X$ dado $Y$ é

$$
f_{X \mid Y}(x \mid y)=\frac{\exp \left(\lambda_{10} x+\lambda_{11} x y+\lambda_{21} x^{2} y\right)}{\int_{a}^{b} \exp \left(\left(\lambda_{10}+\lambda_{11} y\right) u+\lambda_{21} u^{2} y\right) d u}
$$

pois

$$
g_{Y}(y)=\exp \left(\lambda_{0}+\lambda_{01} y\right) \int_{a}^{b} \exp \left(\left(\lambda_{10}+\lambda_{11} y\right) x+\lambda_{21} x^{2} y\right) d x
$$

Por outro lado, a densidade de $Y$ dado $X$ é

$$
g_{Y \mid X}(y \mid x)= \begin{cases}\frac{\left(\lambda_{01}+\lambda_{11} x+\lambda_{21} x^{2}\right) \exp \left(\left(\lambda_{01}+\lambda_{11} x+\lambda_{21} x^{2}\right) y\right)}{\exp \left(\left(\lambda_{01}+\lambda_{11} x+\lambda_{21} x^{2}\right) b\right)-\exp \left(\left(\lambda_{01}+\lambda_{11} x+\lambda_{21} x^{2}\right) a\right)}, & \text { se } \lambda_{01}+\lambda_{11} x+\lambda_{21} x^{2} \neq 0 \\ \frac{1}{b-a}, & \text { se } \lambda_{01}+\lambda_{11} x+\lambda_{21} x^{2}=0\end{cases}
$$

já que

$$
f_{X}(x)= \begin{cases}\frac{\exp \left(\lambda_{0}+\lambda_{10} x\right)\left(\exp \left(\left(\lambda_{01}+\lambda_{11} x+\lambda_{21} x^{2}\right) b\right)-\exp \left(\left(\lambda_{01}+\lambda_{11} x+\lambda_{21} x^{2}\right) a\right)\right)}{\lambda_{01}+\lambda_{11} x+\lambda_{21} x^{2}}, & \text { se } \lambda_{01}+\lambda_{11} x+\lambda_{21} x^{2} \neq 0 \\ \exp \left(\lambda_{0}+\lambda_{10} x\right)(b-a), & \text { se } \lambda_{01}+\lambda_{11} x+\lambda_{21} x^{2}=0\end{cases}
$$

Para a densidade que estamos considerando aqui, dada pela equação (4.9), é evidente que o estudo de $g_{Y \mid X}$ e de $f_{X \mid Y}$ é diferente. Começamos estudando a densidade $g_{Y \mid X}$ pois é mais simples de analisar já que para cada $x \in[a, b], g_{Y \mid X}(\cdot \mid x)$ é uma função constante ou uma função exponencial. É imediato estabelecer o seu tipo de concavidade.

Proposição 4.2.1. $g_{Y \mid X}(\cdot \mid x)$ é uma função convexa para todo $x \in[a, b]$.

Prova: Segue da expressão de $g_{Y \mid X}$.

As seguinte proposição e o seu corolário detalham melhor os possíveis comportamentos de $g_{Y \mid X}(\cdot \mid x)$ segundo $x \in[a, b]$. 
Proposição 4.2.2. Seja $\lambda_{21} \in \mathbb{R}, \lambda_{21} \neq 0$.

1. $g_{Y \mid X}(\cdot \mid x)$ é crescente se, e somente se, $\lambda_{01}+\lambda_{11} x+\lambda_{21} x^{2}>0$;

2. $g_{Y \mid X}(\cdot \mid x)$ é constante se, e somente se, $\lambda_{01}+\lambda_{11} x+\lambda_{21} x^{2}=0$;

3. $g_{Y \mid X}(\cdot \mid x)$ é decrescente se, e somente se, $\lambda_{01}+\lambda_{11} x+\lambda_{21} x^{2}<0$.

Prova: Segue da expressão de $g_{Y \mid X}$.

Corolário 4.2.3. Seja $\lambda_{21} \in \mathbb{R}, \lambda_{21} \neq 0$ e $\lambda_{11}^{2}-4 \lambda_{21} \lambda_{01}>0$. Para $x_{1}=\frac{-\lambda_{11}+\sqrt{\lambda_{11}^{2}-4 \lambda_{21} \lambda_{01}}}{2 \lambda_{21}} e$ $x_{2}=\frac{-\lambda_{11}-\sqrt{\lambda_{11}^{2}-4 \lambda_{21} \lambda_{01}}}{2 \lambda_{21}}$, tais que $\left.x_{1}, x_{2} \in\right] a, b[$ temos que

1. $g_{Y \mid X}(\cdot \mid x)$ é constante para $x=x_{1}$ ou $x=x_{2}$;

2. Se $\lambda_{21}>0$, então

(a) $g_{Y \mid X}(\cdot \mid x)$ é decrescente para $\min \left\{x_{1}, x_{2}\right\}<x<\max \left\{x_{1}, x_{2}\right\}$,

(b) $g_{Y \mid X}(\cdot \mid x)$ é crescente para $\min \left\{x_{1}, x_{2}\right\}>x$ ou $x>\max \left\{x_{1}, x_{2}\right\}$;

3. Se $\lambda_{21}<0$, então

(a) $g_{Y \mid X}(\cdot \mid x)$ é crescente para $\min \left\{x_{1}, x_{2}\right\}<x<\max \left\{x_{1}, x_{2}\right\}$,

(b) $g_{Y \mid X}(\cdot \mid x)$ é decrescente para $\min \left\{x_{1}, x_{2}\right\}>x$ ou $x>\max \left\{x_{1}, x_{2}\right\}$.

Prova: A demonstração é conseqüência da Proposição 4.2.2, observando que $x_{1}$ e $x_{2}$ são as raízes do polinômio $\lambda_{01}+\lambda_{11} x+\lambda_{21} x^{2}$.

Salientamos que $g_{Y \mid X}$ apresenta uma particularidade em comparação as outras densidades condicionais estudadas. Mais especificamente, temos que $g_{Y \mid X}(\cdot \mid x)$ pode ser constante para dois valores diferentes de $x \in[a, b]$, como pode ser visto no corolário anterior.

Uma vez estudada a densidade condicional $g_{Y \mid X}$, continuamos analisando $f_{X \mid Y}$. Pela expressão de $f_{X \mid Y}$, vemos que o estudo do seu comportamento geométrico é equivalente ao estudo do exponente $\left(\lambda_{10}+\lambda_{11} y\right) x+\lambda_{21} x^{2} y$. Assim obtemos o primeiro resultado dado na proposição seguinte. 
Proposição 4.2.4. Sejam $\lambda_{21} \neq 0$ e $y \in[a, b]$.

1. Se $y=0$, então $f_{X \mid Y}(\cdot \mid y)$ é constante.

2. Se $\lambda_{21} y>0$, então

(a) $f_{X \mid Y}(\cdot \mid y)$ é crescente se, e somente se, $\lambda_{10}+\lambda_{11} y+2 \lambda_{21}$ ay $\geq 0$;

(b) $f_{X \mid Y}(\cdot \mid y)$ é decrescente se, e somente se, $\lambda_{10}+\lambda_{11} y+2 \lambda_{21}$ by $\leq 0$;

(c) $f_{X \mid Y}(\cdot \mid y)$ possui um minimizador em $x=\frac{-\lambda_{10}-\lambda_{11} y}{2 \lambda_{21}}$ se, e somente se, $\lambda_{10}+\lambda_{11} y+2 \lambda_{21} a y<0<\lambda_{10}+\lambda_{11} y+2 \lambda_{21} b y$.

3. Se $\lambda_{21} y<0$, então

(a) $f_{X \mid Y}(\cdot \mid y)$ é crescente se, e somente se, $\lambda_{10}+\lambda_{11} y+2 \lambda_{21} b y \geq 0$;

(b) $f_{X \mid Y}(\cdot \mid y)$ é decrescente se, e somente se, $\lambda_{10}+\lambda_{11} y+2 \lambda_{21} a y \leq 0$;

(c) $f_{X \mid Y}(\cdot \mid y)$ possui um maximizador em $x=\frac{-\lambda_{10}-\lambda_{11} y}{2 \lambda_{21}}$ se, e somente se, $\lambda_{10}+\lambda_{11} y+2 \lambda_{21} b y<0<\lambda_{10}+\lambda_{11} y+2 \lambda_{21} a y$.

Prova: Ver a demonstração no Apêndice D.

O seguinte corolário mostra que $f_{X \mid Y}(\cdot \mid y)$ não tem mudança de concavidade.

Corolário 4.2.5. $f_{X \mid Y}(\cdot \mid y)$ possui no máximo um ponto crítico para cada $y \in[a, b]$, e tal ponto é um maximizador ou minimizador.

Prova: Conseqüência imediata da expressão da derivada $\frac{\partial f_{X \mid Y}}{\partial x}(x \mid y)$ obtida na demonstração da Proposição 4.2.4.

Em particular, a Proposição 3.3.1, assim como os correspondentes corolários, podem ser adaptados para $f_{X \mid Y}$. Obtemos dessa forma a seguinte proposição.

Proposição 4.2.6. Seja $P_{f}(x, y)=\lambda_{10}+\lambda_{11} y+2 \lambda_{21} x y$.

1. Se $\min _{(x, y) \in[a, b] \times[a, b]} P_{f}(x, y) \geq 0$, então $f_{X \mid Y}(\cdot \mid y)$ é uma função crescente para todo $y \in[a, b]$;

2. Se $\max _{(x, y) \in[a, b] \times[a, b]} P_{f}(x, y) \leq 0$, então $f_{X \mid Y}(\cdot \mid y)$ é uma função decrescente para todo $y \in[a, b]$. 
Prova: A demonstração é análoga a prova da Proposição 3.3.1.

A seguir, nas Proposições 4.2.7 e 4.2.8 apresentamos as relações entre os coeficientes $\lambda_{10}$, $\lambda_{11}$ e $\lambda_{21}$ segundo a concavidade de $f_{X \mid Y}(\cdot \mid y)$, sendo $f_{X \mid Y}(\cdot \mid y)$ uma função sem ponto crítico, pois esse caso já foi resolvido na Proposição 4.2.4.

Proposição 4.2.7. Seja $\lambda_{21} \in \mathbb{R}, \lambda_{21} \neq 0$ e $y \in[a, b]$. Se $\lambda_{21} y>0$ então $f_{X \mid Y}(\cdot \mid y)$ é convexa .

Prova: A demonstração resulta imediata a partir da expressão da segunda derivada de $f_{X \mid Y}(\cdot \mid y)$ obtida na prova da Proposição 4.2.4.

Proposição 4.2.8. Sejam $\lambda_{21} \in \mathbb{R}, y \in[a, b]$ e $\lambda_{21} y<0$.

1. Se $f_{X \mid Y}(\cdot \mid y)$ for crescente, então $f_{X \mid Y}(\cdot \mid y)$ é convexa se, e somente se, $2 \lambda_{21} y+\left(\lambda_{10}+\lambda_{11} y+2 \lambda_{21} b y\right)^{2} \geq 0$

2. Se $f_{X \mid Y}(\cdot \mid y)$ for crescente, então $f_{X \mid Y}(\cdot \mid y)$ é côncava se, e somente se, $2 \lambda_{21} y+\left(\lambda_{10}+\lambda_{11} y+2 \lambda_{21} a y\right)^{2} \leq 0 ;$

3. Se $f_{X \mid Y}(\cdot \mid y)$ for decrescente, então $f_{X \mid Y}(\cdot \mid y)$ é convexa se, e somente se, $2 \lambda_{21} y+\left(\lambda_{10}+\lambda_{11} y+2 \lambda_{21} a y\right)^{2} \geq 0 ;$

4. Se $f_{X \mid Y}(\cdot \mid y)$ for decrescente, então $f_{X \mid Y}(\cdot \mid y)$ é côncava se, e somente se, $2 \lambda_{21} y+\left(\lambda_{10}+\lambda_{11} y+2 \lambda_{21} b y\right)^{2} \leq 0$.

Prova: Análoga a demonstração da Proposição 4.1.10.

A seguir vemos que $f_{X \mid Y}(\cdot \mid y)$ possui a mesma concavidade para todo $y \in[a, b]$.

Proposição 4.2.9. Sejam $\lambda_{21} \in \mathbb{R}, \lambda_{21} \neq 0$ e $y_{0} \in[a, b]$.

1. Se $f_{X \mid Y}\left(\cdot \mid y=y_{0}\right)$ é convexa, então $f_{X \mid Y}(\cdot \mid y)$ é convexa para todo $y \in[a, b]$;

2. Se $f_{X \mid Y}\left(\cdot \mid y=y_{0}\right)$ é côncava, então $f_{X \mid Y}(\cdot \mid y)$ é côncava para todo $y \in[a, b]$.

Prova: Demonstração análoga à prova do Corolário 4.1.11.

Finalizamos o estudo da densidade conjunta $h$ dada na equação (4.9) com a análise dos seus pontos críticos. 
Proposição 4.2.10. A densidade h possui no máximo dois pontos críticos dados por

$$
\left(x_{1}, y_{1}\right)=\left(\frac{-\lambda_{11}+\sqrt{\lambda_{11}^{2}-4 \lambda_{21} \lambda_{01}}}{2 \lambda_{21}},-\frac{\lambda_{10} \sqrt{\lambda_{11}^{2}-4 \lambda_{21} \lambda_{01}}}{\lambda_{11}^{2}-4 \lambda_{21} \lambda_{01}}\right)
$$

e

$$
\left(x_{2}, y_{2}\right)=\left(\frac{-\lambda_{11}-\sqrt{\lambda_{11}^{2}-4 \lambda_{21} \lambda_{01}}}{2 \lambda_{21}}, \frac{\lambda_{10} \sqrt{\lambda_{11}^{2}-4 \lambda_{21} \lambda_{01}}}{\lambda_{11}^{2}-4 \lambda_{21} \lambda_{01}}\right) .
$$

Tais pontos são pontos de sela de $h$.

Prova: Ver a demonstração no Apêndice D.

Exemplificamos, na Figura 4.2.1, uma densidade conjunta com os seguintes dois pontos de sela $(1,-1 / 2)$ e $(-1,1 / 2)$ no domínio $[-2,2] \times[-2,2]$.

\section{Figura 4.2.1}

Densidade $h(x, y)=\exp \left(-5,1+x-y+x^{2} y\right)$, para $(x, y) \in[-2,2] \times[-2,2]$.

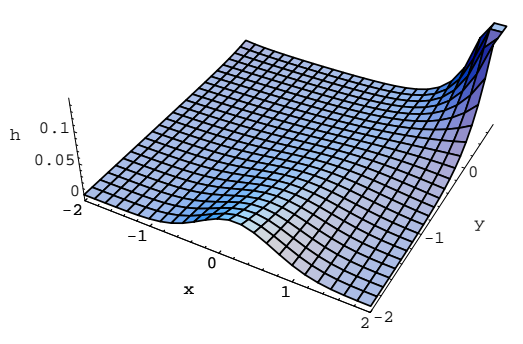

(a) Densidade conjunta $h$.

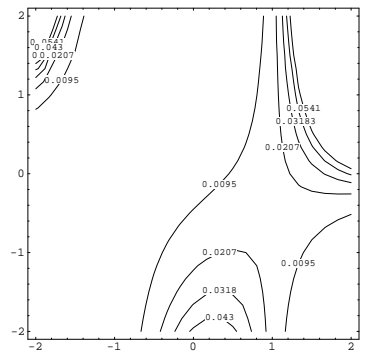

(b) Curvas de nível da densidade conjunta.

Concluímos observando que todos os resultados enunciados e demonstrados no presente capítulo foram feitos por nós. O conteúdo da seção 4.1.1 foi apresentado em "Third Brazilian Conference on Statistical Modelling in Insurance and Finance", ver Fernández e Kolev (2007a). 


\section{Capítulo 5}

\section{Medidas de dependência}

No presente capítulo expomos diversas medidas de dependência que existem na bibliografia. Começamos com a função de dependência local que, em alguns casos, permite inferir outras propriedades de associação entre as variáveis aleatórias. Logo continuamos a apresentação fazendo distinção entre medidas de dependência positiva e negativa. No caso de associação positiva estamos mensurando quão relacionados são os valores altos de uma das variáveis com os valores altos da outra. Enquanto que no caso negativo obtemos informação sobre a relação entre os valores altos de uma variável e os valores baixos da outra. Finalmente, aplicamos os conceitos e resultados mostrados às densidades estudadas nos capítulos anteriores.

\subsection{Função de dependência local}

Começamos a análise das medidas de associação com a função de dependência local. Para as densidades que estamos estudando neste trabalho é simples calcular tal função. Por sua vez, a função de dependência local fornece informações sobre outras medidas de dependência mais complexas de calcular (no caso das densidades aqui estudadas), como por exemplo a covariância. 
Definição 5.1.1. Seja $h$ uma densidade bivariada tal que existe sua segunda derivada mista $\frac{\partial^{2} h}{\partial x \partial y}$. A função $\gamma_{h}$ definida no domínio de $h$ como

$$
\gamma_{h}(x, y)=\frac{\partial^{2} \ln (h)}{\partial x \partial y}(x, y)
$$

é denominada função de dependência local $(f d l)$.

Holland e Wang (1987) motivaram a $f d l$ como a versão contínua do "odds ratio" ou razão dos produtos cruzados definida na equação (6.1). Por outro lado, em Jones (1996), a $f d l$ é motivada como uma localização do coeficiente de correlação.

A $f d l$ possui diversas propriedades interessantes, aqui citamos somente algumas.

Proposição 5.1.2 (Holland e Wang (1987)). Seja $h$ uma densidade que possui segunda derivada mista e sejam $f_{X \mid Y}$ e $g_{Y \mid X}$ as correspondentes densidades condicionais. A fdl $\gamma_{h}$ satisfaz as seguintes propriedades.

1. $\gamma_{h}$ é finita no domínio;

2. $\gamma_{h}$ é nula se, e somente se, $X$ e $Y$ são independentes;

3. $\gamma_{h}(x, y)=\gamma_{f_{X \mid Y}}(x, y)=\gamma_{g_{Y \mid X}}(x, y)$ ("medida de dependência livre das marginais").

A $f d l$ pode ser usada para medir a dependência entre duas variáveis tanto quando a intensidade como a direção de dependência mudam em diferentes regiões do domínio. Um exemplo interessante, fornecido em Jones (1996), é dado na Figura 5.1.1 onde apresentamos a $f d l$ da densidade bivariada de Cauchy dada pela expressão $1 /\left(\pi\left(1+x^{2}+y^{2}\right)^{3 / 2}\right)$. Na figura podemos observar regiões com diferentes valores de dependência assim como com diferentes sinais.

Para as densidades conjuntas consideradas no presente trabalho, isto é,

$$
h_{1}(x, y)=\exp \left(\lambda_{0}+\lambda_{10} x+\lambda_{01} y+\lambda_{11} x y+\lambda_{20} x^{2}+\lambda_{02} y^{2}\right), \quad(x, y) \in[a, b] \times[a, b]
$$

e

$$
h_{2}(x, y)=\exp \left(\lambda_{0}+\lambda_{10} x+\lambda_{01} y+\lambda_{11} x y+\lambda_{21} x^{2} y\right), \quad(x, y) \in[a, b] \times[a, b]
$$

é imediato obter as correspondentes $f d l$

$$
\gamma_{h_{1}}(x, y)=\lambda_{11} \quad \text { e } \quad \gamma_{h_{2}}(x, y)=\lambda_{11}+2 \lambda_{21} x .
$$


Figura 5.1.1

Curvas de nível da $f d l$ da distribuição de Cauchy.

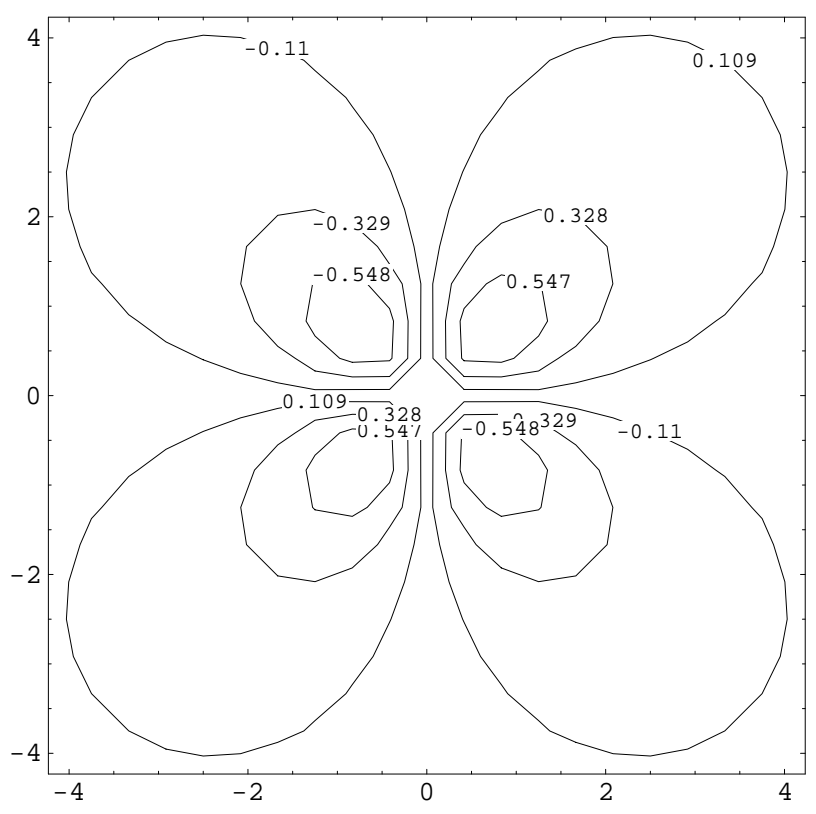

Para $h_{1}$ dada em (5.2) vemos que a dependência local é constante. A densidade $h_{1}$ é um membro da família de densidades bivariadas com $f d l$ constante. Em Jones (1998) é provado que se uma densidade $h$ possui $f d l$ constante igual a $\xi$, então $h$ deve ser da forma

$$
h(x, y)=\alpha(x ; \xi) \beta(y ; \xi) \exp (\xi x y)
$$

sendo $\alpha(x ; \xi)$ e $\beta(y ; \xi)$ funções não negativas arbitrárias.

Por outro lado, para $h_{2}$ dada na equação (5.3) a $f d l$ é uma função linear na variável $x$, isto implica que temos regiões do domínio de $h_{2}$ com diferente intensidade e direção de depêndencia. Por exemplo, para $h_{2}$ com domínio $[0,1] \times[0,1]$ e parâmetros $\lambda_{10}=0=\lambda_{01}, \lambda_{11}=-0,5 \mathrm{e}$ $\lambda_{21}=0,5$ obtemos $\gamma_{h_{2}}=x-0,5$. Na Figura 5.1 .2 mostramos as curvas de nível de $\gamma_{h_{2}}$. Podemos observar que para $x<0,5$ a dependência é negativa e para $x>0,5$ é positiva. 
Figura 5.1.2

Curvas de nível para $(x, y) \in[0,1] \times[0,1]$ da $f d l$ da densidade $h(x, y)=\exp \left(0,194-0,5 x y+0,5 x^{2} y\right)$.

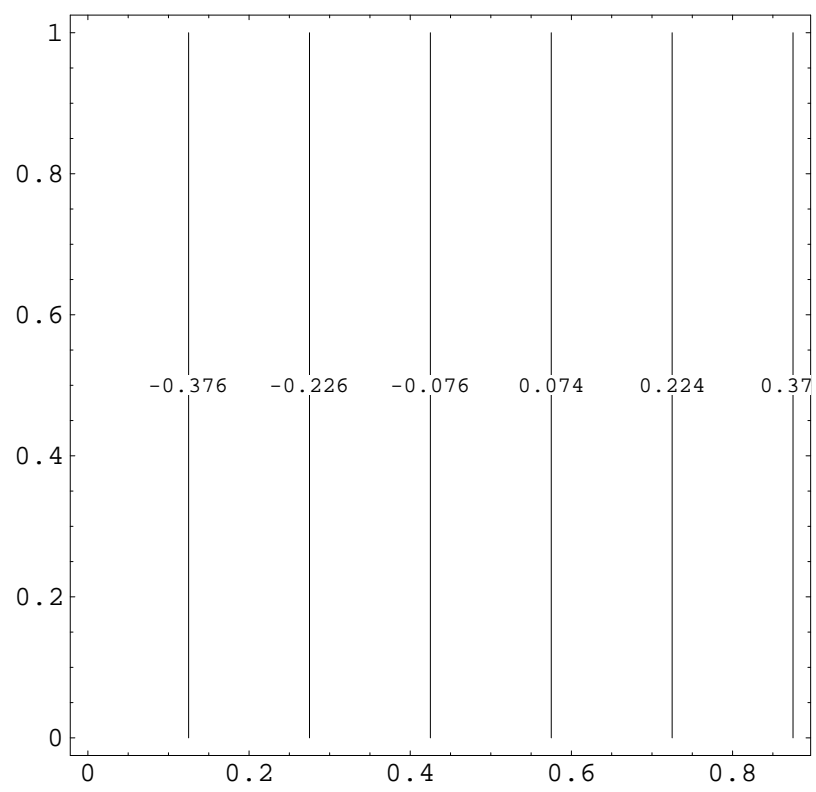

\subsection{Dependência positiva}

A seguir apresentamos medidas de dependência positiva entre duas variáveis. Começamos com a medida mais fraca (no sentido do Teorema 5.2.5) que compara a probabilidade de qualquer quadrante em relação à densidade conjunta com a probabilidade no caso de independência.

Definição 5.2.1. Sejam $X$ e $Y$ duas variáveis aleatórias. Dizemos que $X$ e $Y$ são positivamente dependentes em quadrante (PQD) se

$$
\mathbb{P}[X \leq x, Y \leq y] \geq \mathbb{P}[X \leq x] \mathbb{P}[Y \leq y] \text { para todo } x, y
$$

As seguintes medidas de dependência são relações mais fortes do que PQD. 
Definição 5.2.2. Sejam $X$ e $Y$ duas variáveis aleatórias. Dizemos que

- $Y$ é decrescente na cauda esquerda em X $(\operatorname{LTD}(Y \mid X))$ se

$$
\mathbb{P}[Y \leq y \mid X \leq x]
$$

é decrescente em $x$ para todo $y$;

- $Y$ é crescente na cauda direita em X $(\operatorname{RTI}(Y \mid X))$ se

$$
\mathbb{P}[Y>y \mid X>x]
$$

é crescente em $x$ para todo $y$;

- $Y$ é estocasticamente crescente em X $(\mathrm{SI}(Y \mid X))$ se

$$
\mathbb{P}[Y>y \mid X=x]
$$

é crescente em $x$ para todo $y$.

Em Lehmann (1966) o conceito SI é denominado "dependência positiva de regressão", dando assim uma intuição mais clara da relação que apresentam duas variáveis quando são SI.

O conceito de associação positiva entre duas variáveis dado a seguir é uma medida de dependência mais forte do que a medida usual de covariância.

Definição 5.2.3. Sejam $X$ e $Y$ duas variáveis aleatórias. Dizemos que $X$ e $Y$ são associadas positivamente $(\mathrm{AP}(X, Y))$ se

$$
\operatorname{Cov}\left(l_{1}(X, Y), l_{2}(X, Y)\right) \geq 0
$$

sendo $l_{1}, l_{2}: \mathbb{R}^{2} \mapsto \mathbb{R}$ funções não decrescentes em cada argumento, tais que existem $\mathbb{E}\left[l_{1}(X, Y) l_{2}(X, Y)\right], \mathbb{E}\left[l_{1}(X, Y)\right]$ e $\mathbb{E}\left[l_{2}(X, Y)\right]$.

Em particular, se duas variáveis são associadas positivamente, então possuem covariância não negativa. Por último, temos o conceito de densidade totalmente positiva. 
Definição 5.2.4. Sejam $X$ e $Y$ variáveis com densidade conjunta $h(x, y)$. $h$ é chamada totalmente positiva (TP) se o determinante

$$
\left|\begin{array}{ll}
h\left(x_{1}, y_{1}\right) & h\left(x_{1}, y_{2}\right) \\
h\left(x_{2}, y_{1}\right) & h\left(x_{2}, y_{2}\right)
\end{array}\right| \geq 0
$$

para $x_{1}<x_{2}$ e $y_{1}<y_{2}$.

O seguinte teorema ordena hierarquicamente as medidas de dependência definidas anteriormente.

Teorema 5.2.5 (Barlow e Proschan (1975)). Sejam $X$ e $Y$ variáveis aleatórias. A seguinte seqüência de implicações é válida. TP $\Rightarrow S I(Y \mid X) \Rightarrow R T I(Y \mid X) \Rightarrow A P(X, Y) \Rightarrow$ $P Q D(X, Y)$. A seqüência continua valendo se substituirmos $R T I(Y \mid X)$ por $\operatorname{LTD}(Y \mid X)$.

Todas as medidas de dependência apresentadas podem ser relacionadas via o Teorema 5.2.5 e o seguinte resultado.

Teorema 5.2.6 (Holland e Wang (1987)). Uma densidade bivariada h é TP se, e somente se, $\gamma_{h} \geq 0$.

Como $\gamma_{h}=0$ é equivalente à independência e $\gamma_{h}>0$ é equivalente a TP estrita, Holland e Wang (1987) sugerem interpretar o conceito de TP como "mais dependência positiva do que independência". A vantagem da $f d l$ em relação a TP é que $\gamma_{h}$, além de qualificar a dependência, como a TP, também quantifica.

A seguir aplicamos os resultados anteriores para $h_{1}$ e $h_{2}$. A densidade $h_{1}$, dada na equação (5.2) satisfaz que

$$
\lambda_{11} \geq 0 \Leftrightarrow T P .
$$

Em particular concluímos que o sinal de $\lambda_{11}$ coincide com o sinal da covariância entre $X$ e $Y$.

No caso da densidade $h_{2}$ dada na equação (5.3) obtemos que

$$
\left(\lambda_{21}>0 \text { e } \lambda_{11}+2 \lambda_{21} a \geq 0\right) \quad \text { ou } \quad\left(\lambda_{21}<0 \text { e } \lambda_{11}+2 \lambda_{21} b \geq 0\right) \quad \Leftrightarrow \quad T P .
$$

O exposto anteriormente pode ser combinado com os resultados dos capítulos 3 e 4 para determinar os possíveis coeficientes da densidade. Por exemplo, se conhecemos que a $f d l$ é constante e as variáveis são positivamente correlacionadas, então utilizamos a expressão dada por (5.2) com $\lambda_{11}>0$ como aproximação da densidade conjunta. Logo aplicamos os resultados do capítulo 3 ou 4 segundo conhecermos o comportamento das marginais ou das condicionais. 


\subsection{Dependência negativa}

Nesta seção damos algumas medidas de dependência negativa. Os conceitos são simétricos aos expostos na parte de dependência positiva.

Definição 5.3.1. Sejam $X$ e $Y$ duas variáveis aleatórias. Dizemos que $X$ e $Y$ são negativamente dependentes em quadrante (NDQ) se

$$
\mathbb{P}[X \leq x, Y \leq y] \leq \mathbb{P}[X \leq x] \mathbb{P}[Y \leq y] \text { para todo } x, y
$$

A definição anterior pode ser relacionada com a medida de dependência positiva PQD como mostra o seguinte lema.

Lema 5.3.2. $N Q D(X, Y)$ se, e somente se, $P Q D(X,-Y)$.

Prova: Conseqüência da relação

$$
\mathbb{P}(X \leq x,-Y \leq-y)=\mathbb{P}(X \leq x, Y>y)=\mathbb{P}(X \leq x)-\mathbb{P}(X \leq x, Y \leq y)
$$

A medida NQD é equivalente com a definição de variáveis negativamente associadas dada a seguir. A demonstração da equivalência pode ser vista em Joag-Dev e Proschan (1983).

Definição 5.3.3. Sejam $X$ e $Y$ duas variáveis aleatórias. Dizemos que $X$ e $Y$ são associadas negativamente $(\mathrm{AN}(X, Y))$ se

$$
\operatorname{Cov}\left(l_{1}(X), l_{2}(Y)\right) \leq 0
$$

sendo $l_{1}, l_{2}: \mathbb{R} \mapsto \mathbb{R}$ funções não decrescentes tais que existem $\mathbb{E}\left[l_{1}(X) l_{2}(Y)\right], \mathbb{E}\left[l_{1}(X)\right]$ e $\mathbb{E}\left[l_{2}(Y)\right]$.

Em particular, se duas variáveis são associadas negativamente, então possuem covariância não positiva. Observamos que existe uma diferência importante na definição de variáveis AP e AN. No caso de associação positiva as funções $l_{1}$ e $l_{2}$ da definição possuem $\mathbb{R}^{2}$ como domínio, enquanto que no caso de associação negativa o domínio das funções $l_{1}$ e $l_{2}$ é $\mathbb{R}$. São desconsideradas as funções de domínio $\mathbb{R}^{2}$ no caso de $\mathrm{AN}$ para evitar a condição $0 \geq \operatorname{Cov}(X, X)=\operatorname{Var}(X)$. A diferência mencionada, faz com que a relação entre AN e AP seja num único sentido, isto é, podemos inferir a propriedade de AN a partir de AP, mas não ao contrário. No lema seguinte mostramos a relação entre AP e AN. 
Lema 5.3.4. Se $A P(X,-Y)$, então $A N(X, Y)$.

Prova: Demonstração imediata a apartir das definições de AP e AN.

Definição 5.3.5. Sejam $X$ e $Y$ duas variáveis aleatórias. Dizemos que

- $Y$ é crescente na cauda esquerda em X $(\operatorname{LTI}(Y \mid X))$ se

$$
\mathbb{P}[Y \leq y \mid X \leq x]
$$

é crescente em $x$ para todo $y$;

- $Y$ é decrescente na cauda direita em X $(\operatorname{RTD}(Y \mid X))$ se

$$
\mathbb{P}[Y>y \mid X>x]
$$

é decrescente em $x$ para todo $y$;

- $Y$ é estocasticamente decrescente em X $(\mathrm{SD}(Y \mid X))$ se

$$
\mathbb{P}[Y>y \mid X=x]
$$

é decrescente em $x$ para todo $y$.

Assim como no Lema 5.3.2, podemos relacionar as definições anteriores com as medidas análogas de dependência positiva.

Lema 5.3.6. Sejam $X$ e $Y$ duas variáveis aleatórias.

1. $\operatorname{LTD}(-Y \mid X)$ se, e somente se, $\operatorname{LTI}(Y \mid X)$;

2. $R T I(-Y \mid X)$ se, e somente se, $R T D(Y \mid X)$;

3. $S I(-Y \mid X)$ se, e somente se, $S D(Y \mid X)$.

Prova: A demonstração segue da relação $\mathbb{P}(Y \leq y \mid X \leq x)=1-\mathbb{P}(-Y<-y \mid X \leq x)$.

A última definição de dependência negativa que apresentamos é simétrica à propriedade de TP. 
Definição 5.3.7. Sejam $X$ e $Y$ variáveis com densidade conjunta $h(x, y)$. $h$ é chamada totalmente negativa $(\mathrm{TN})$ se o determinante

$$
\left|\begin{array}{ll}
h\left(x_{1}, y_{1}\right) & h\left(x_{1}, y_{2}\right) \\
h\left(x_{2}, y_{1}\right) & h\left(x_{2}, y_{2}\right)
\end{array}\right| \leq 0
$$

para $x_{1}<x_{2}$ e $y_{1}<y_{2}$.

Lema 5.3.8. Se $h^{+}$denota a densidade conjunta de $X$ e $Y$ e $h^{-}$a de $X$ e $-Y$, então $h^{+}$é TP se, e somente se, $h^{-}$é $T N$.

Prova: Conseqüência da igualdade $h^{+}(x, y)=h^{-}(x,-y)$.

As relações vistas entre as medidas de dependência positiva e negativa permitem obter uma ordenação simétrica à dada no Teorema 5.2.5 mas para o caso de dependência negativa.

Teorema 5.3.9. Sejam $X$ e $Y$ variáveis aleatórias. A seguinte seqüência de implicações é válida. $T N \Rightarrow S D(Y \mid X) \Rightarrow R T D(Y \mid X) \Rightarrow A N(X, Y) \Rightarrow N Q D(X, Y)$. A seqüência continua valendo se substituirmos $R T D(Y \mid X)$ por $\operatorname{LTI}(Y \mid X)$.

Prova: A demonstração é conseqüência do Teorema 5.2.5, do Lema 5.3.6 e da equivalência entre AN e NQD.

Concluímos a apresentação das relações entre as diferentes medidas de dependência negativas com o seguinte teorema.

Teorema 5.3.10 (Holland e Wang (1987)). Uma densidade bivariada h é TN se, e somente se, $\gamma_{h} \leq 0$.

Assim como no caso de dependência positiva, analisamos a seguir a $f d l$ das densidades $h_{1}$ e $h_{2}$ para obter implicações sobre as medidas de dependência negativa, em particular, covariância negativa.

Seja $h_{1}$ a densidade dada na equação (5.2), então

$$
\lambda_{11} \leq 0 \Leftrightarrow T N .
$$

Em particular, concluímos que o sinal de $\lambda_{11}$ é contrário ao sinal da covariância entre $X$ e $Y$. 
No caso da densidade $h_{2}$ dada na equação (5.3) obtemos que

$$
\left(\lambda_{21}>0 \text { e } \lambda_{11}+2 \lambda_{21} b \leq 0\right) \quad \text { ou } \quad\left(\lambda_{21}<0 \text { e } \lambda_{11}+2 \lambda_{21} a \leq 0\right) \quad \Leftrightarrow \quad T N
$$

Como já foi observado no fim da seção 5.1, as medidas de dependência negativa aqui apresentadas podem ser combinadas com a informação das marginais ou das condicionais para determinar os possíveis valores dos coeficientes da densidade bivariada. 


\section{Capítulo 6}

\section{Variáveis discretas}

O objetivo deste capítulo é mostrar a influência do comportamento marginal e condicional sobre uma função de probabilidade conjunta de variáveis aleatórias discretas. Apresentamos os resultados para variáveis bivariadas com marginais definidas num conjunto de dois elementos e de três elementos. Expomos o estudo do comportamento marginal, do comportamento condicional e da medida de dependência dada pela razão dos produtos cruzados.

\subsection{Tabelas $2 \times 2$}

Começamos assumindo que as variáveis $X$ e $Y$ tomam valores no conjunto $\{0,1\}$. A função de probabilidade conjunta é apresentada na seguinte tabela, sendo $p_{i j}=\mathbb{P}(X=i, Y=j)$, $p_{i \bullet}=\mathbb{P}(X=i)$ e $p_{\bullet}=\mathbb{P}(Y=j)$, para $i, j=0,1$.

\section{Tabela 6.1.1}

Função de probabilidade conjunta.

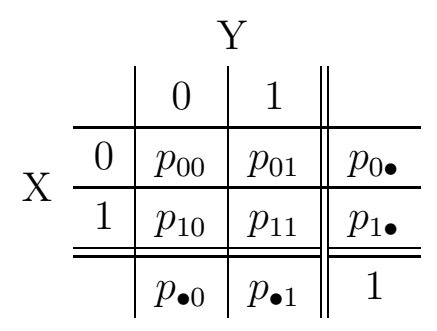


As correspondentes funções de probabilidades marginais podem ser constantes, crescentes ou decrescentes. Exemplificamos tais comportamentos na Figura 6.1.2.

\section{Figura 6.1.2}

Possíveis comportamentos da função de probabilidade $f_{X}$.

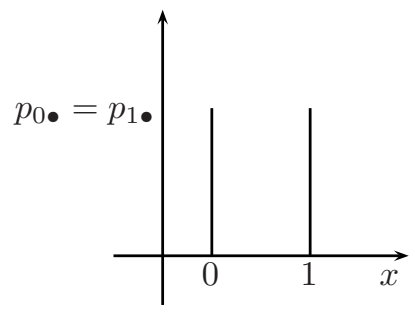

$f_{X}$ constante

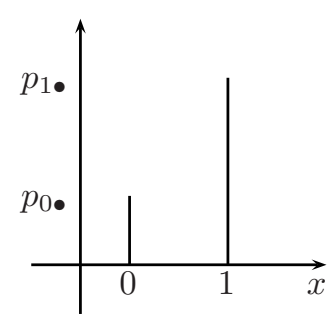

$f_{X}$ crescente

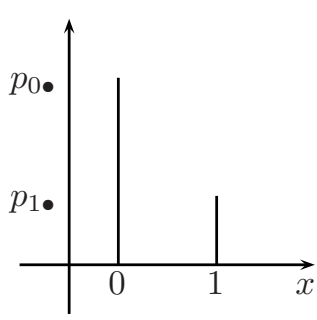

$f_{X}$ decrescente

A partir das igualdades

$$
\begin{aligned}
& p_{0 \bullet}=p_{00}+p_{01}, \\
& p_{1 \bullet}=p_{10}+p_{11}, \\
& p_{\bullet 0}=p_{00}+p_{10}, \\
& p_{\bullet 1}=p_{01}+p_{11}
\end{aligned}
$$

é imediato obter a Tabela 6.1 .3 com as relações entre os valores $p_{i j}$, para $i, j=0,1$, segundo a natureza geométrica das marginais.

Na Tabela 6.1 .3 vemos que se $X$ e $Y$ possuem função de probabilidade crescente, então a probabilidade conjunta deve satisfazer

$$
1=p_{00}+p_{11}+p_{10}+p_{01}, \quad\left|p_{10}-p_{01}\right|<p_{11}-p_{00} \quad \text { e } \quad p_{00}<p_{11}
$$

Assim como nos casos de variáveis contínuas, podemos analisar a influência das funções de probabilidades condicionais sobre a probabilidade conjunta. Em particular, assumimos conhecido o comportamento geométrico de $X$ dado $Y$, ou seja, de $f_{X \mid Y}$. A seguir damos uma lista dos possíveis comportamentos de $f_{X \mid Y}$ e das respectivas probabilidades $p_{i j}, i, j=0,1$. 


\section{Tabela 6.1.3}

Relações entre as probabilidades conjuntas $p_{i j}$, para $i, j=0,1$, segundo o comportamento geométrico das marginais.

\begin{tabular}{|c|c|c|c|}
\hline & $f_{X}^{c}$ & $f_{X}^{i}$ & $f_{X}^{d}$ \\
\hline$g_{Y}^{c}$ & $p_{00}=p_{11}=p_{10}=p_{01}=\frac{1}{4}$ & $\begin{array}{c}p_{01}<p_{10} \\
p_{00}=p_{01}+p_{11}-p_{10} \\
p_{10} \leq p_{01}+p_{11} \\
1=p_{00}+p_{11}+p_{10}+p_{01}\end{array}$ & $\begin{array}{c}p_{10}<p_{01} \\
p_{11}=p_{00}+p_{10}-p_{01} \\
p_{01} \leq p_{00}+p_{10} \\
1=p_{00}+p_{11}+p_{10}+p_{01}\end{array}$ \\
\hline$g_{Y}^{i}$ & $\begin{array}{c}p_{10}<p_{01} \\
p_{00}=p_{10}+p_{11}-p_{01} \\
p_{01} \leq p_{10}+p_{11} \\
1=p_{00}+p_{11}+p_{10}+p_{01}\end{array}$ & $\begin{array}{c}p_{00}<p_{11} \\
\left|p_{10}-p_{01}\right|<p_{11}-p_{00} \\
1=p_{00}+p_{11}+p_{10}+p_{01}\end{array}$ & $\begin{array}{c}p_{10}<p_{01} \\
\left|p_{11}-p_{00}\right|<p_{01}-p_{10} \\
1=p_{00}+p_{11}+p_{10}+p_{01}\end{array}$ \\
\hline$g_{Y}^{d}$ & $\begin{array}{c}p_{01}<p_{10} \\
p_{11}=p_{00}+p_{01}-p_{10} \\
p_{10} \leq p_{00}+p_{01} \\
1=p_{00}+p_{11}+p_{10}+p_{01}\end{array}$ & $\begin{array}{c}p_{01}<p_{10} \\
\left|p_{11}-p_{00}\right|<p_{10}-p_{01} \\
1=p_{00}+p_{11}+p_{10}+p_{01}\end{array}$ & $\begin{array}{c}p_{00}>p_{11} \\
\left|p_{10}-p_{01}\right|<p_{00}-p_{11} \\
1=p_{00}+p_{11}+p_{10}+p_{01}\end{array}$ \\
\hline
\end{tabular}

\section{Proposição 6.1.1.}

1. $f_{X \mid Y}(\cdot \mid y=0)$ e $f_{X \mid Y}(\cdot \mid y=1)$ são funções constantes se, e somente se, $p_{00}=p_{10}$ e $p_{01}=p_{11}$;

2. $f_{X \mid Y}(\cdot \mid y=0)$ é uma função constante e $f_{X \mid Y}(\cdot \mid y=1)$ é uma função crescente se, e somente se, $p_{00}=p_{10}$ e $p_{01}<p_{11}$;

3. $f_{X \mid Y}(\cdot \mid y=0)$ é uma função constante e $f_{X \mid Y}(\cdot \mid y=1)$ é uma função decrescente se, $e$ somente se, $p_{00}=p_{10}$ e $p_{01}>p_{11}$;

4. $f_{X \mid Y}(\cdot \mid y=0)$ e $f_{X \mid Y}(\cdot \mid y=1)$ são funções crescentes se, e somente se, $p_{00}<p_{10}$ e $p_{01}<p_{11}$;

5. $f_{X \mid Y}(\cdot \mid y=0)$ é uma função crescente e $f_{X \mid Y}(\cdot \mid y=1)$ é uma função decrescente se, $e$ somente se, $p_{00}<p_{10}$ e $p_{01}>p_{11}$;

6. $f_{X \mid Y}(\cdot \mid y=0)$ e $f_{X \mid Y}(\cdot \mid y=1)$ são funções decrescentes se, e somente se, $p_{00}>p_{10} e$ $p_{01}>p_{11}$.

Prova: Demonstração imediata a partir da definição de $f_{X \mid Y}$. 
Agora apresentamos um exemplo da utilização dos resultados até aqui mostrados. Assumimos que a função de probabilidade de $Y$ é crescente e que a função de probabilidade de $X$ dado $Y$ é decrescente para $y \in\{0,1\}$. Isto implica que as probabilidades conjuntas devem satisfazer as seguintes relações

$$
p_{00}+p_{10}<p_{01}+p_{11}, \quad p_{00}>p_{10}, \quad p_{01}>p_{11} \quad \text { e } \quad 1=p_{00}+p_{11}+p_{10}+p_{01}
$$

Uma possível representação é dada na Tabela 6.1.4.

\section{Tabela 6.1.4}

Representação de uma função de probabilidade conjunta sendo $g_{Y}$ crescente e $f_{X \mid Y}(\cdot \mid y)$ decrescente para $y \in\{0,1\}$.

\begin{tabular}{c|c|c||c} 
& \multicolumn{2}{c|}{$\mathrm{Y}$} \\
& 0 & 1 & \\
\hline 0 & 0,25 & 0,5 & 0,75 \\
\hline 1 & 0,2 & 0,05 & 0,25 \\
\hline \hline & 0,45 & 0,55 & 1
\end{tabular}

\begin{tabular}{c|c|c}
$X$ & 0 & 1 \\
\hline$f_{X \mid Y}(\cdot \mid y=0)$ & 0,56 & 0,4 \\
\hline$f_{X \mid Y}(\cdot \mid y=1)$ & 0,9 & 0,1
\end{tabular}

Concluímos o estudo das variáveis discretas com valores no conjunto $\{0,1\}$, analisando a razão dos produtos cruzados. A razão dos produtos cruzados, denotada por $\theta$, mede o grau de associação entre duas variáveis dadas numa tabela de $2 \times 2$. É definida por

$$
\theta \stackrel{\text { def }}{=} \frac{p_{00} p_{11}}{p_{10} p_{01}}
$$

Quando $\theta=1$ as variáveis $X$ e $Y$ são independentes. Se $\theta>1$, então os eventos $(X=0, Y=0)$ e $(X=1, Y=1)$ são os mais prováveis de acontecer. Ao contrário, se $0 \leq \theta<1$, então os eventos $(X=0, Y=1)$ e $(X=1, Y=0)$ têm mais chances de serem observados. A seguir mostramos limitantes para $\theta$ segundo o comportamento geométrico de $f_{X \mid Y}$. 


\section{Proposição 6.1.2.}

1. Se $f_{X \mid Y}(\cdot \mid y=0)$ e $f_{X \mid Y}(\cdot \mid y=1)$ são funções constantes, então $\theta=1$;

2. Se $f_{X \mid Y}(\cdot \mid y=0)$ é uma função constante e $f_{X \mid Y}(\cdot \mid y=1)$ é uma função crescente, então $\theta>1$;

3. Se $f_{X \mid Y}(\cdot \mid y=0)$ é uma função constante e $f_{X \mid Y}(\cdot \mid y=1)$ é uma função decrescente, então $\theta<1$;

4. Se $f_{X \mid Y}(\cdot \mid y=0)$ e $f_{X \mid Y}(\cdot \mid y=1)$ são funções crescentes, então $\frac{p_{00}}{p_{10}}<\theta<\frac{p_{11}}{p_{01}}$;

5. Se $f_{X \mid Y}(\cdot \mid y=0)$ é uma função crescente e $f_{X \mid Y}(\cdot \mid y=1)$ é uma função decrescente, então $\theta<1$;

6. Se $f_{X \mid Y}(\cdot \mid y=0)$ e $f_{X \mid Y}(\cdot \mid y=1)$ são funções decrescentes, entãa $\frac{p_{11}}{p_{01}}<\theta<\frac{p_{00}}{p_{10}}$.

Prova: A demonstração segue da Proposição 6.1.1 e da equação (6.1).

Finalizamos a presente seção observando que para o exemplo dado na Tabela 6.1.4, obtemos que $\frac{p_{11}}{p_{01}}=0,1<\theta=0,125<1,25=\frac{p_{00}}{p_{10}}$.

\subsection{Tabelas $3 \times 3$}

Nesta seção estudamos as variáveis $X$ e $Y$ no caso em que assumem valores num conjunto de três elementos, em particular, no conjunto $\{0,1,2\}$. A função de probabilidade conjunta é dada na Tabela 6.2.1.

\section{Tabela 6.2.1}

Função de probabilidade conjunta.

\begin{tabular}{c|c|c|c||c} 
& \multicolumn{5}{c||}{$\mathrm{Y}$} \\
& 0 & 1 & 2 & \\
\hline 0 & $p_{00}$ & $p_{01}$ & $p_{02}$ & $p_{0 \bullet}$ \\
\hline 1 & $p_{10}$ & $p_{11}$ & $p_{12}$ & $p_{1 \bullet}$ \\
\hline 2 & $p_{20}$ & $p_{21}$ & $p_{22}$ & $p_{2 \bullet}$ \\
\hline \hline & $p_{\bullet}$ & $p_{\bullet 1}$ & $p_{\bullet 2}$ & 1
\end{tabular}


Dentre todos os comportamentos possíveis das funções de probabilidade marginais, apenas estudamos os mostrados na Figura 6.2.2. Nos restringimos a esses casos por considerá-los os mais relevantes.

\section{Figura 6.2.2}

Possíveis comportamentos da função de probabilidade $f_{X}$.

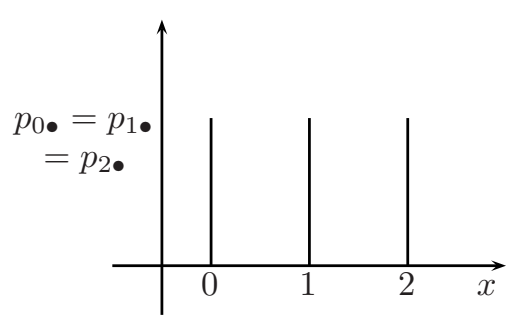

$f_{X}$ constante

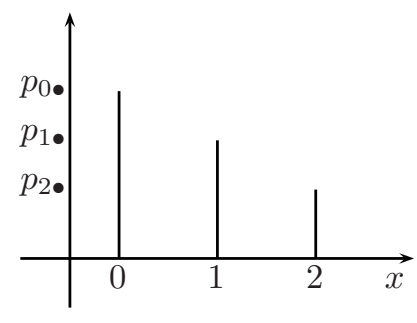

$f_{X}$ decrescente

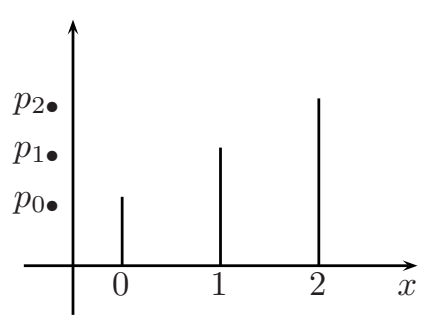

$f_{X}$ crescente

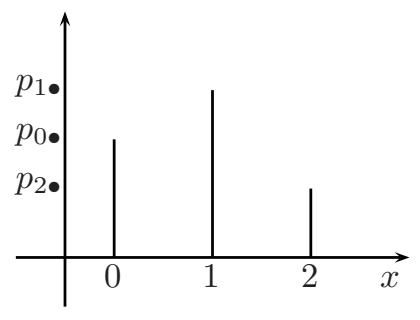

$f_{X}$ unimodal

A partir das igualdades

$$
\begin{array}{lll}
p_{\bullet 0}=p_{00}+p_{10}+p_{20}, & p_{\bullet 1}=p_{01}+p_{11}+p_{21}, & p_{\bullet 2}=p_{02}+p_{12}+p_{22}, \\
p_{0 \bullet}=p_{00}+p_{01}+p_{02}, & p_{1 \bullet}=p_{10}+p_{11}+p_{12}, & p_{2 \bullet}=p_{20}+p_{21}+p_{22}
\end{array}
$$

e das definições de marginais crescentes, decrescentes, constantes e unimodais, podemos obter relações simples entre as probabilidades $p_{i j}$, para $i, j=0,1,2$, segundo o comportamento marginal. A título de exemplo, mostramos os passos para estudar o caso em que $f_{X}$ e $g_{Y}$ são funções crescentes.

$$
f_{X}^{i} \text { e } g_{Y}^{i} \Leftrightarrow\left\{\begin{array}{l}
p_{00}+p_{10}+p_{20}<p_{01}+p_{11}+p_{21}<p_{02}+p_{12}+p_{22} \\
p_{00}+p_{01}+p_{02}<p_{10}+p_{11}+p_{12}<p_{20}+p_{21}+p_{22} .
\end{array}\right.
$$


Somando os termos das duas seqüências de desigualdades obtemos que

$$
\left\{\begin{array}{l}
2 p_{00}+p_{10}+p_{02}+p_{20}+p_{01}<p_{01}+p_{10}+2 p_{11}+p_{12}+p_{21} \\
p_{01}+p_{10}+2 p_{11}+p_{12}+p_{21}<p_{02}+p_{20}+p_{21}+p_{12}+2 p_{22}
\end{array} .\right.
$$

Portanto $2 p_{00}+p_{01}+p_{10}<p_{21}+p_{12}+2 p_{22}$.

A seguinte proposição enuncia as relações obtidas para as diferentes combinações de comportamentos de $f_{X}$ e $g_{Y}$.

\section{Proposição 6.2.1.}

1. Se $f_{X}$ e $g_{Y}$ são funções constantes, então $p_{i j}=\frac{1}{9}$ para todo $i, j=0,1,2$;

2. Se $f_{X}$ é uma função constante e $g_{Y}$ é uma função crescente, então $p_{10}+p_{20}<p_{01}+p_{02}$;

3. Se $f_{X}$ é uma função constante e $g_{Y}$ é uma função decrescente, então $p_{10}+p_{20}>p_{01}+p_{02}$;

4. Se $f_{X}$ é uma função constante e $g_{Y}$ é uma função unimodal, então $p_{12}+p_{10}<p_{01}+p_{21}$;

5. Se $f_{X}$ e $g_{Y}$ são funções crescentes, então $2 p_{00}+p_{01}+p_{10}<p_{21}+p_{12}+2 p_{22}$;

6. Se $f_{X}$ é uma função crescente e $g_{Y}$ é uma função decrescente, então $p_{01}+2 p_{02}+p_{12}<2 p_{20}+p_{21}+p_{10}$

7. Se $f_{X}$ é uma função crescente e $g_{Y}$ é uma função unimodal, então $2 p_{00}+p_{10}+2 p_{02}+p_{12}<2 p_{11}+p_{01}+3 p_{21}$

8. Se $f_{X}$ e $g_{Y}$ são funções decrescentes, então $2 p_{00}+p_{01}+p_{10}>p_{21}+p_{12}+2 p_{22}$;

9. Se $f_{X}$ é uma função decrescente e $g_{Y}$ é uma função unimodal, então $3 p_{01}+p_{21}+2 p_{11}>p_{10}+p_{12}+2 p_{20}+2 p_{22}$;

10. Se $f_{X}$ e $g_{Y}$ são funções unimodais, então

$$
2 p_{00}+2 p_{02}+2 p_{20}+2 p_{22}<4 p_{11}+p_{12}+p_{21}+p_{10}+p_{01} \text {. }
$$

Prova: Demonstração análoga à realizada anteriormente para o caso em que ambas marginais são crescentes.

Agora passamos a estudar as funções de probabilidades condicionais. Especificamente, analisamos a variável $X$ dado $Y$ com função de probabilidade $f_{X \mid Y}$. Novamente nos limitamos aos casos de funções de probabilidades constantes, crescentes, decrescentes e unimodais. Para cada $j \in\{0,1,2\}$ é imediato obter as relações listadas a seguir. 


\section{Proposição 6.2.2.}

1. $f_{X \mid Y}(\cdot \mid y=j)$ é uma função constante se, e somente se, $p_{0 j}=p_{1 j}=p_{2 j}$;

2. $f_{X \mid Y}(\cdot \mid y=j)$ é uma função crescente se, e somente se, $p_{0 j}<p_{1 j}<p_{2 j}$;

3. $f_{X \mid Y}(\cdot \mid y=j)$ é uma função decrescente se, e somente se, $p_{0 j}>p_{1 j}>p_{2 j}$;

4. $f_{X \mid Y}(\cdot \mid y=j)$ é uma função unimodal se, e somente se, $p_{0 j}<p_{1 j}$ e $p_{2 j}<p_{1 j}$.

Prova: Segue da definição de $f_{X \mid Y}$.

A título de exemplo, damos na Tabela 6.2.3 uma representação para uma função de probabilidade conjunta com $g_{Y}$ crescente, $f_{X \mid Y}(\cdot \mid y=0)$ e $f_{X \mid Y}(\cdot \mid y=1)$ decrescentes e $f_{X \mid Y}(\cdot \mid y=2)$ unimodal.

\section{Tabela 6.2 .3}

Representação de uma função de probabilidade conjunta sendo $g_{Y}$ crescente, $f_{X \mid Y}(\cdot \mid y)$ decrescente para $y \in\{0,1\}$ e unimodal para $y=2$.

\begin{tabular}{|c|c|c|c|c|c|}
\hline & \multicolumn{4}{|c|}{$\mathrm{Y}$} \\
\hline & & 0 & 1 & 2 & \\
\hline & 0 & 0,1 & 0,2 & 0,1 & 0,4 \\
\hline X & 1 & 0,05 & 0,1 & 0,2 & 0,35 \\
\hline & 2 & 0,01 & 0,09 & 0,15 & 0,25 \\
\hline & & 0,16 & 0,39 & 0,45 & 1 \\
\hline
\end{tabular}

\begin{tabular}{c|c|c|c}
$X$ & 0 & 1 & 2 \\
\hline$f_{X \mid Y}(\cdot \mid y=0)$ & 0,625 & 0,3125 & 0,0625 \\
\hline$f_{X \mid Y}(\cdot \mid y=1)$ & 0,513 & 0,256 & 0,231 \\
\hline$f_{X \mid Y}(\cdot \mid y=2)$ & 0,222 & 0,445 & 0,333
\end{tabular}

A razão dos produtos cruzados $\theta$ pode ser utilizada neste caso também. Para isso calculamos $\theta$ para cada subtabela de $2 \times 2$. Assim, temos que calcular as nove razões seguintes.

$$
\begin{aligned}
& \theta_{00,11}=\frac{p_{00} p_{11}}{p_{01} p_{10}}, \quad \theta_{00,12}=\frac{p_{00} p_{12}}{p_{10} p_{02}}, \quad \theta_{00,21}=\frac{p_{00} p_{21}}{p_{20} p_{01}}, \\
& \theta_{00,22}=\frac{p_{00} p_{22}}{p_{20} p_{02}}, \quad \theta_{01,12}=\frac{p_{01} p_{12}}{p_{11} p_{02}}, \quad \theta_{01,22}=\frac{p_{01} p_{22}}{p_{21} p_{02}}, \\
& \theta_{10,21}=\frac{p_{10} p_{21}}{p_{20} p_{11}}, \quad \theta_{10,22}=\frac{p_{10} p_{22}}{p_{20} p_{12}}, \quad \theta_{11,22}=\frac{p_{11} p_{22}}{p_{21} p_{12}} .
\end{aligned}
$$


Existem quatro subtabelas (ou quatro $\theta$ ) elementares a partir das quais podem ser obtidas todas as razões dos produtos cruzados. Observemos que

$$
\begin{aligned}
\theta_{00,11} \theta_{01,12} & =\theta_{00,12}, \\
\theta_{00,11} \theta_{10,21} & =\theta_{00,21}, \\
\theta_{01,12} \theta_{11,22} & =\theta_{01,22}, \\
\theta_{10,21} \theta_{11,22} & =\theta_{10,22}, \\
\theta_{00,11} \theta_{01,12} \theta_{11,22} \theta_{10,21} & =\theta_{00,22} .
\end{aligned}
$$

Ou seja, $\theta_{00,11}, \theta_{01,12}, \theta_{11,22}$ e $\theta_{10,21}$ são as razões dos produtos cruzados elementares, pois a partir delas podem ser obtidas todas as outras razões. Agora estudamos a influência do comportamento geométrico de $f_{X \mid Y}$ sobre as razões dos produtos cruzados elementares. Da definição $\theta_{00,11}=\frac{p_{00} p_{11}}{p_{01} p_{10}}$, obtemos que $\theta_{00,11}$ está relacionado com as funções de probabilidade $f_{X \mid Y}(\cdot \mid y=0)$ e $f_{X \mid Y}(\cdot \mid y=1)$. De forma similar podemos ver que cada razão elementar é influenciada por somente duas das três funções condicionais $f_{X \mid Y}$. Mais especificamente,

$$
\begin{array}{ccccccc}
f_{X \mid Y}(\cdot \mid y=0) & \text { e } & f_{X \mid Y}(\cdot \mid y=1) & \rightsquigarrow & \theta_{00,11} & \text { e } & \theta_{10,21} \\
f_{X \mid Y}(\cdot \mid y=1) & \text { e } & f_{X \mid Y}(\cdot \mid y=2) & \rightsquigarrow & \theta_{01,12} & \text { e } & \theta_{11,22} .
\end{array}
$$

Logo, os limitantes das razões elementares segundo o comportamento de $f_{X \mid Y}$ podem ser resumidos na forma apresentada na Tabela 6.2.4.

Por exemplo, na Tabela 6.2 .4 vemos que se $f_{X \mid Y}(\cdot \mid y)$ é crescente para $y=0$, constante para $y=1$ e decrescente para $y=2$, então as quatro razões elementares são menores do que um, isto é,

$$
\begin{array}{ll}
\theta_{00,11}<1, & \theta_{10,21}<1, \\
\theta_{11,22}<1, & \theta_{01,12}<1 .
\end{array}
$$

Isso implica que as nove razões dos produtos cruzados de $X$ e $Y$ são menores do que um. Essa propriedade é interpretada em Yule e Kendall (1950) como dependência ou associação negativa entre $X$ e $Y$.

Observamos que para o exemplo dado na Tabela 6.2 .3 as correspondentes razões dos 
Tabela 6.2.4

Limitantes das razões dos produtos cruzados elementares segundo o comportamento geométrico de $f_{X \mid Y}$.

\begin{tabular}{|c|c|c|c|c|c|c|}
\hline & \multicolumn{6}{|c|}{$f_{X \mid Y}(\cdot \mid y=1)$} \\
\hline & constante & & crescente & decrescente & & unimodal \\
\hline$f_{X \mid Y}(\cdot \mid y=0)$ & $\theta_{00,11}=1$ & & $\theta_{00,11}>1$ & $\theta_{00,11}<1$ & & $\theta_{00,11}>1$ \\
\hline constante & $\theta_{10,21}=1$ & & $\theta_{10,21}>1$ & $\theta_{10,21}<1$ & & $\theta_{10,21}<1$ \\
\hline$f_{X \mid Y}(\cdot \mid y=0)$ & $\theta_{00,11}<1$ & & $<\theta_{00,11}<\frac{p_{11}}{p_{01}}$ & $\theta_{00,11}<1$ & $\frac{p_{00}}{p_{10}}$ & $<\theta_{00,11}<\frac{p_{11}}{p_{01}}$ \\
\hline crescente & $\theta_{10,21}<1$ & $\frac{p_{10}}{p_{20}}$ & $<\theta_{10,21}<\frac{p_{21}}{p_{11}}$ & $\theta_{10,21}<1$ & & $\theta_{10,21}<1$ \\
\hline$f_{X \mid Y}(\cdot \mid y=0)$ & $\theta_{00,11}>1$ & & $\theta_{00,11}>1$ & $\frac{p_{11}}{p_{01}}<\theta_{00,11}<\frac{p_{00}}{p_{10}}$ & & $\theta_{00,11}>1$ \\
\hline decrescente & $\theta_{10,21}>1$ & & $\theta_{10,21}>1$ & $\frac{p_{21}}{p_{11}}<\theta_{10,21}<\frac{p_{10}}{p_{20}}$ & $\frac{p_{21}}{p_{11}}$ & $<\theta_{10,21}<\frac{p_{10}}{p_{20}}$ \\
\hline$f_{X \mid Y}(\cdot \mid y=0)$ & $\theta_{00,11}<1$ & $\frac{p_{00}}{p_{10}}$ & $<\theta_{00,11}<\frac{p_{11}}{p_{01}}$ & $\theta_{00,11}<1$ & $\frac{p_{00}}{p_{10}}$ & $<\theta_{00,11}<\frac{p_{11}}{p_{01}}$ \\
\hline unimodal & $\theta_{10,21}>1$ & & $\theta_{10,21}>1$ & $\frac{p_{21}}{p_{11}}<\theta_{10,21}<\frac{p_{10}}{p_{20}}$ & $\frac{p_{21}}{p_{11}}$ & $<\theta_{10,21}<\frac{p_{10}}{p_{20}}$ \\
\hline$f_{X \mid Y}(\cdot \mid y=2)$ & $\theta_{11,22}=1$ & & $\theta_{11,22}<1$ & $\theta_{11,22}>1$ & & $\theta_{11,22}>1$ \\
\hline constante & $\theta_{01,12}=1$ & & $\theta_{01,12}<1$ & $\theta_{01,12}>1$ & & $\theta_{01,12}<1$ \\
\hline$f_{X \mid Y}(\cdot \mid y=2)$ & $\theta_{11,22}>1$ & $\frac{p_{11}}{p_{21}}$ & $<\theta_{11,22}<\frac{p_{22}}{p_{12}}$ & $\theta_{11,22}>1$ & & $\theta_{11,22}>1$ \\
\hline crescente & $\theta_{01,12}>1$ & $\frac{p_{01}}{p_{11}}$ & $<\theta_{01,12}<\frac{p_{12}}{p_{02}}$ & $\theta_{01,12}>1$ & $\frac{p_{01}}{p_{11}}$ & $<\theta_{01,12}<\frac{p_{12}}{p_{02}}$ \\
\hline$f_{X \mid Y}(\cdot \mid y=2)$ & $\theta_{11,22}<1$ & & $\theta_{11,22}<1$ & $\frac{p_{22}}{p_{12}}<\theta_{11,22}<\frac{p_{11}}{p_{11}}$ & $\frac{p_{22}}{p_{12}}$ & $<\theta_{11,22}<\frac{p_{11}}{p_{21}}$ \\
\hline decrescente & $\theta_{01,12}<1$ & & $\theta_{01,12}<1$ & $\frac{p_{12}}{p_{02}}<\theta_{01,12}<\frac{p_{01}}{p_{11}}$ & & $\theta_{01,12}<1$ \\
\hline$f_{X \mid Y}(\cdot \mid y=2)$ & $\theta_{11,22}<1$ & & $\theta_{11,22}<1$ & $\frac{p_{22}}{p_{12}}<\theta_{11,22}<\frac{p_{11}}{p_{21}}$ & $\frac{p_{22}}{p_{12}}$ & $<\theta_{11,22}<\frac{p_{11}}{p_{21}}$ \\
\hline unimodal & $\theta_{01,12}>1$ & $\frac{p_{01}}{p_{11}}$ & $<\theta_{01,12}<\frac{p_{12}}{p_{02}}$ & $\theta_{01,12}>1$ & $\frac{p_{01}}{p_{11}}$ & $<\theta_{01,12}<\frac{p_{12}}{p_{02}}$ \\
\hline
\end{tabular}

produtos cruzados elementares e os seus limitantes são

$$
\begin{aligned}
0,5 & =\frac{p_{11}}{p_{01}}<\theta_{00,11}=1<\frac{p_{00}}{p_{10}}=2 ; \quad 0,9=\frac{p_{21}}{p_{11}}<\theta_{10,21}=4,5<\frac{p_{10}}{p_{20}}=5 ; \\
0,75 & =\frac{p_{22}}{p_{12}}<\theta_{11,22}=0,83<\frac{p_{11}}{p_{21}}=1,1 ; \quad 1<\theta_{01,12}=5 .
\end{aligned}
$$

Os limitantes de $\theta_{00,11}, \theta_{10,21}, \theta_{11,22}$ e $\theta_{01,12}$ foram obtidos a partir da Tabela 6.2.4 usando que $f_{X \mid Y}(\cdot \mid y)$ é decrescente para $y \in\{0,1\}$ e unimodal para $y=2$.

Finalizamos notando que as Proposições 6.1.1, 6.1.2, 6.2.1 e 6.2.2, assim como a Tabela 6.1.3 e a Tabela 6.2.4, são resultados originais. 


\section{Capítulo 7}

\section{Conclusões e estudos futuros}

No presente trabalho mostramos que uma densidade bivariada contínua pode ser aproximada, num domínio limitado, pela exponencial de um polinômio. Logo, assumindo que é conhecido o comportamento geométrico das densidades marginais ou condicionais, determinamos os possíveis valores dos coeficientes quando a densidade é dada por

$$
h(x, y)=\exp \left(\lambda_{0}+\lambda_{10} x+\lambda_{01} y+\lambda_{11} x y+\lambda_{20} x^{2}+\lambda_{02} y^{2}\right), \quad(x, y) \in[a, b] \times[a, b]
$$

ou por

$$
h(x, y)=\exp \left(\lambda_{0}+\lambda_{10} x+\lambda_{01} y+\lambda_{11} x y+\lambda_{21} x^{2} y\right), \quad(x, y) \in[a, b] \times[a, b]
$$

sendo $[a, b] \subset \mathbb{R}$. Também estudamos as relações entre os coeficientes e algumas medidas de dependência. Finalizamos mostrando a influência do comportamento marginal e condicional sobre variáveis bivariadas discretas. Concluindo que a natureza geométrica das marginais, assim como das condicionais, define famílias de densidades bivariadas.

Acreditamos que a técnica aqui exposta, ou seja, a representação de uma densidade bivariada segundo o comportamento das marginais, pode ter muitas aplicações assim como diversas extensões. Os resultados apresentados podem ser ampliados, por exemplo, incluindo a informação dos primeiros momentos marginais e mistos. Também pode ser estudada a ordem de convergência da aproximação e o grau dos polinômios que aproximam a densidade conjunta.

Nos resultados expostos vimos que a informação das marginais define um conjunto de possíveis densidades, especificamente de possíveis coeficientes. Uma questão que surge naturalmente é escolher um dos elementos desse conjunto. Acerca desse tema já estamos pesquisando 
sobre a determinação de densidades bivariadas de máxima entropia segundo a natureza das marginais.

Finalizamos observando que a metodologia aqui apresentada pode ser estendida para densidades conjuntas de mais variáveis. Os resultados sobre a aproximação, exibidos no capítulo 2, continuam valendo para qualquer número de variáveis. Claramente, a complexidade de trabalhar em dimensões maiores é dada pelo incremento dos coeficientes a serem classificados. 


\section{Referências bibliográficas}

Ang, D. D., Gorenflo, R. e Trong, D. D. (1999). A multi-dimensional Hausdorff moment problem: regularization by finite moments. Journal for Analysis and its Applications 18(1): 13-25.

Arnold, B. C., Castillo, E. e Sarabia, J. M. (1999). Conditional Specification of Statistical Models. Springer Series in Statistics. Springer.

Barlow, R. C. e Proschan, F. (1975). Statistical Theory of Reliability and Life Testing: Probability Models. Holt, Reinhart and Winston Inc.

Barndorff-Nielsen, O. (1978). Information and Exponential Families in Statistical Theory. New York: Wiley.

Cuadras, C. M., Fortiana, J., Rodríguez-Lallena, J. (Eds.) (2002). Distributions With Given Marginals and Statistical Modelling. Kluwer Academic Publishers.

Fernández, M. e Kolev, N. (2006). Classifying a bivariate joint density. Program Book of the 69th Annual Meeting of the Institute of Mathematical Statistics and the 5th International Symposium on Probability and its Applications in conjuction with the X Brazilian School of Probability, 62.

Fernández, M. e Kolev, N. (2007a). Bivariate density approximation under the marginal and conditional information. Proceedings of the Third Brazilian Conference on Statistical Modelling in Insurance and Finance, (Eds. Fernandes, C., Schmidli, H. e Kolev, N.), 284-287. 
Fernández, M. e Kolev, N. (2007b). Bivariate density characterization by the geometry of marginals. Aceito para publicação em Journal of Economic Quality Control.

Holland, P. W. e Wang, Y. J. (1987). Dependence function for continuous bivariate densities. Communications in Statistics-Theory and Methods 16(3): 863-876.

Joag-Dev, K. e Proschan, F. (1983). Negative association of random variables, with applications. The Annals of Statistics. 11(1): 286-295.

Johnson, N. L. e Kotz, S. (1972). Distributions in Statistics: Continuous Multivariate Distributions. Wiley.

Jones, M. C. (1996). The local dependence function. Biometrika 83(4): 899-904.

Jones, M. C. (1998). Constant local dependence. Journal of Multivariate Analysis 64: $148-155$.

Knill, O. (1997) On Hausdorff's moment problem in higher dimensions. Preprint.

Kolev, N. e Fernández, M. (2006). Bivariate density classification by the geometry of marginals. Proceedings of the 10th International Congress on Insurance: Mathematics and Economics, 73-74.

Kotz, S. e van Dorp, R. (2002). A versatile bivariate distribution on a bounded domain: another look at the product moment correlation. Journal of Applied Statistics 29(8): 1165-1179.

Lages Lima, E. (2002). Curso de Análise. Volume 1, décima edição. Projeto Euclides.

Lages Lima, E. (2003). Espaços Métricos. Terceira edição. Projeto Euclides.

Lancaster, H. O. (1958). The structure of bivariate distributions. Ann. Math. Statist. 29: $719-736$.

Lehmann, E. L. (1966). Some concepts of dependence. Ann. Math. Statist. 43: 1137-1153.

Luenberger, D. G. (1989). Linear and Nonlinear Programming . Segunda edição. AddisonWesley. 
Nguyen, H. T. e Rogers, G. S. (1989). Fundamentals of Mathematical Statistics. Volume II: Statistical Inference. Springer.

Romano, Joseph P. and Siegel, Andrew F. (1986) Counterexamples in Probability and Statistics. Chapman \& Hall.

Rudin, W. (1976). Principles of Mathematical Analysis. International Series in Pure and Applied Mathematics. McGraw-Hill. Terceira edição.

Shohat, J. A. e Tamarkin, J. D. (1943). The Problem of Moments. American Mathematical Society.

Yule, G. U. e Kendall, M. G. (1950). An Introduction to the Theory of Statistics. New York: Hafner Publishing Company. 


\section{Apêndice A}

\section{Demonstrações de resultados auxiliares}

O presente capítulo do anexo é destinado a enunciar e provar alguns resultados gerais que são necessários em diferentes partes do trabalho.

Proposição A.1. A função $K: \mathbb{R} \mapsto \mathbb{R}$ definida por

$$
K(z) \stackrel{\text { def }}{=} \begin{cases}\frac{\exp (z)}{\exp (z)-1}-\frac{1}{z}, & \text { se } z \neq 0 \\ \frac{1}{2}, & \text { se } z=0\end{cases}
$$

é contínua e estritamente crescente.

Prova: Começamos provando a continuidade. Para $z \neq 0$ a função é contínua por ser soma e quociente de funções contínuas não nulas. Para $z=0$ provamos a continuidade calculando o correspondente limite, isto é $\lim _{z \rightarrow 0} K(z)=K(0)$. Como tal limite é do tipo $\frac{0}{0}$ aplicamos duas vezes a regra de L'Hospital e obtemos que 


$$
\begin{aligned}
\lim _{z \rightarrow 0} K(z) & =\lim _{z \rightarrow 0}\left(\frac{\exp (z)}{\exp (z)-1}-\frac{1}{z}\right)=\lim _{z \rightarrow 0} \frac{\exp (z)(z-1)+1}{z(\exp (z)-1)} \\
& =\lim _{z \rightarrow 0} \frac{\frac{d}{d z}(\exp (z)(z-1)+1)}{\frac{d}{d z}(z(\exp (z)-1))}=\lim _{z \rightarrow 0} \frac{\exp (z) z}{\exp (z)(1+z)-1} \\
& =\lim _{z \rightarrow 0} \frac{\frac{d}{d z}(\exp (z) z)}{\frac{d}{d z}(\exp (z)(1+z)-1)}=\lim _{z \rightarrow 0} \frac{1+z}{2+z} \\
& =\frac{1}{2} .
\end{aligned}
$$

Portanto podemos concluir que $K$ é uma função contínua para $z \in \mathbb{R}$.

Para demonstrar que é uma função crescente trabalhamos com a derivada de $K$. No ponto $z=0$ a derivada deve ser calculada pela própia definição como limite. Não apresentamos aqui os detalhes pois é uma conta similar à prova de continuidade, mas tendo que aplicar quatro vezes a regra de L'Hospital. Tal derivada está dada por

$$
\frac{d K}{d z}(z)= \begin{cases}\frac{1}{z^{2}}-\frac{\exp (z)}{(\exp (z)-1)^{2}}, & \text { se } z \neq 0 \\ \frac{1}{12}, & \text { se } z=0\end{cases}
$$

Veremos primeiro que a derivada da função $K$ é positiva no conjunto dos números positivos, ou seja, $\frac{d K}{d z}(z)>0$ para todo $\left.z \in\right] 0, \infty[$. A seguir apresentamos as operações algébricas que permitem provar o procurado. Seja $z \in] 0, \infty[$ e $i$ um número natural $(i \in \mathbb{N}) \operatorname{com} i \geq 3$, então temos que

$$
\begin{aligned}
2^{i-1} & >i \\
2^{i-1} \frac{z^{i-1}}{2^{i-1} i !} & >i \frac{z^{i-1}}{2^{i-1} i !} \\
\frac{z^{i-1}}{i !} & >\frac{\left(\frac{z}{2}\right)^{i-1}}{(i-1) !} .
\end{aligned}
$$


Logo, para todo $n \in \mathbb{N}$ tal que $n \geq 4$, vale que

$$
\begin{aligned}
& \sum_{i=4}^{n} \frac{z^{i-1}}{i !}>\sum_{i=3}^{n-1} \frac{\left(\frac{z}{2}\right)^{i}}{i !} \\
& \lim _{n \rightarrow \infty} \sum_{i=4}^{n} \frac{z^{i-1}}{i !} \geq \lim _{n \rightarrow \infty} \sum_{i=3}^{n-1} \frac{\left(\frac{z}{2}\right)^{i}}{i !} \\
& \frac{1}{z} \lim _{n \rightarrow \infty} \sum_{i=4}^{n} \frac{z^{i}}{i !} \geq \lim _{n \rightarrow \infty} \sum_{i=3}^{n-1} \frac{\left(\frac{z}{2}\right)^{i}}{i !}
\end{aligned}
$$

Aplicando a relação $1+\frac{z}{2}+\frac{z^{2}}{6}>1+\frac{z}{2}+\frac{z^{2}}{8}$ na desigualdade anterior obtemos que

$$
\begin{aligned}
1+\frac{z}{2}+\frac{z^{2}}{6}+\frac{1}{z} \lim _{n \rightarrow \infty} \sum_{i=4}^{n} \frac{z^{i}}{i !} & >1+\frac{z}{2}+\frac{z^{2}}{8}+\lim _{n \rightarrow \infty} \sum_{i=3}^{n-1} \frac{\left(\frac{z}{2}\right)^{i}}{i !} \\
\frac{1}{z} \lim _{n \rightarrow \infty} \sum_{i=1}^{n} \frac{z^{i}}{i !} & >\lim _{n \rightarrow \infty} \sum_{i=0}^{n-1} \frac{\left(\frac{z}{2}\right)^{i}}{i !} \\
\frac{1}{z}(\exp (z)-1) & >\exp \left(\frac{z}{2}\right) \\
\left(\frac{\exp (z)-1}{z}\right)^{2} & >\exp (z) \\
\frac{1}{z^{2}} & >\frac{\exp (z)}{(\exp (z)-1)^{2}} \\
\frac{1}{z^{2}}-\frac{\exp (z)}{(\exp (z)-1)^{2}} & >0
\end{aligned}
$$

Portanto provamos que a derivada da função $K$ é positiva para $z \in] 0, \infty[$. Agora precisamos obter o mesmo resultado para $z \in]-\infty, 0[$. Isto será realizado mostrando que a derivada de 
$K$ é uma função par, ou seja $\frac{d K}{d z}(z)=\frac{d K}{d z}(-z)$.

$$
\begin{aligned}
\exp (z)-2+\exp (-z) & =\exp (z)-2+\exp (-z) \\
\exp (z)(1-2 \exp (-z)+\exp (-2 z)) & =\exp (-z)(\exp (2 z)-2 \exp (z)+1) \\
\exp (z)(1-\exp (-z))^{2} & =\exp (-z)(\exp (z)-1)^{2} \\
\frac{\exp (z)}{(\exp (z)-1)^{2}} & =\frac{\exp (-z)}{(\exp (-z)-1)^{2}} \\
\frac{1}{z^{2}}-\frac{\exp (z)}{(\exp (z)-1)^{2}} & =\frac{1}{(-z)^{2}}-\frac{\exp (-z)}{(\exp (-z)-1)^{2}} \\
\frac{d K}{d z}(z) & =\frac{d K}{d z}(-z) .
\end{aligned}
$$

Portanto podemos concluir que a derivada de $K$ é positiva para todo número real $z, \log K$ é estritamente crescente.

Corolário A.2. A função $K_{c}: \mathbb{R} \mapsto \mathbb{R}$ definida por

$$
K_{c}(z) \stackrel{\text { def }}{=} \begin{cases}\frac{c \exp (c z)}{\exp (c z)-1}-\frac{1}{z}, & \text { se } z \neq 0 \\ \frac{c}{2}, & \text { se } z=0\end{cases}
$$

é contínua e estritamente crescente para $c \in \mathbb{R}$ e $c \neq 0$.

Prova: Seja $c \neq 0$. Para todo $z \in \mathbb{R}$ temos que $K_{c}(z)=c K(c z)$. Logo o corolário é conseqüência imediata da proposição anterior, Proposição A.1.

Corolário A.3. A função $K_{c}$ é limitada como segue

$$
0<K_{c}(z)<c \quad \text { para todo } \quad z \in \mathbb{R}
$$

Prova: Começamos observando que

$$
\lim _{z \rightarrow-\infty} K_{c}(z)=0 \quad \text { e } \quad \lim _{z \rightarrow \infty} K_{c}(z)=c .
$$

Por outro lado, como a função $K_{c}$ é estritamente crescente concluímos que

$$
\lim _{z \rightarrow-\infty} K_{c}(z)<K_{c}(z)<\lim _{z \rightarrow \infty} K_{c}(z)
$$


Juntando os dois resultados obtemos as desigualdades procuradas, isto é

$$
0<K_{c}(z)<c
$$

para qualquer $z$ número real.

Lema A.4. A função K possui primeira e segunda derivada contínuas.

Prova: Na prova da Proposição A.1 vimos que existe a primeira derivada de $K$ para todo $z \in \mathbb{R}$. Agora vamos provar que existe a segunda derivada e isto implica a continuidade da primeira derivada.

Como temos que

$$
\frac{d K}{d z}(z)= \begin{cases}\frac{1}{z^{2}}-\frac{\exp (z)}{(\exp (z)-1)^{2}}, & \text { se } z \neq 0 \\ \frac{1}{12}, & \text { se } z=0\end{cases}
$$

para $z \neq 0$ obtemos

$$
\frac{d^{2} K}{d z^{2}}(z)=\frac{2-2 \exp (3 z)+\exp (z)\left(-6+z^{3}\right)+\exp (2 z)\left(6+z^{3}\right)}{z^{3}(\exp (z)-1)^{3}},
$$

ou seja, para tudo $z \neq 0$ existe a segunda derivada da função $K$. Para calcular a segunda derivada na origem utilizamos a própria definição, obtendo assim que

$$
\begin{aligned}
\left.\frac{d^{2} K}{d z^{2}}(z)\right|_{z=0} & =\lim _{z \rightarrow 0} \frac{\frac{1}{z^{2}}-\frac{\exp (z)}{(\exp (z)-1)^{2}}-\frac{1}{12}}{z} \\
& =\lim _{z \rightarrow 0} \frac{(\exp (z)-1)^{2}\left(12-z^{2}\right)-12 \exp (z) z^{3}}{12 z^{3}(\exp (z)-1)^{2}} \\
& =0 .
\end{aligned}
$$

O limite acima foi calculado aplicando três vezes a regra de L'Hospital. Assim obtemos a expressão da segunda derivada em todo o domínio,

$$
\frac{d^{2} K}{d z^{2}}(z)= \begin{cases}\frac{2-2 \exp (3 z)+\exp (z)\left(-6+z^{3}\right)+\exp (2 z)\left(6+z^{3}\right)}{z^{3}(\exp (z)-1)^{3}}, & \text { se } z \neq 0 \\ 0, & \text { se } z=0\end{cases}
$$


A continuidade é imediata da expressão anterior para $z \neq 0$. Na origem temos que ver que o seguinte limite

$$
\lim _{z \rightarrow 0} \frac{2-2 \exp (3 z)+\exp (z)\left(-6+z^{3}\right)+\exp (2 z)\left(6+z^{3}\right)}{z^{3}(\exp (z)-1)^{3}}
$$

é zero. Isso pode ser obtido aplicando seis vezes a regra de L'Hospital. Portanto concluimos que a função $K$ possui primeira e segunda derivada contínuas.

Lema A.5. A segunda derivada da função $K$ é impar, isto é $\frac{d^{2} K}{d z^{2}}(z)=-\frac{d^{2} K}{d z^{2}}(-z)$, e somente é nula na origem.

Prova: Começamos analisando a propriedade da segunda derivada ser uma função ímpar. Na prova da Proposição A.1 vimos que a primeira derivada é par, ou seja, $\frac{d K}{d z}(z)=\frac{d K}{d z}(-z)$. Logo, é imediato ver que a segunda derivada satisfaz $\frac{d^{2} K}{d z^{2}}(z)=-\frac{d^{2} K}{d z^{2}}(-z)$.

Trabalhemos agora o fato de somente se anular no ponto $z=0$. Por ser uma função ímpar é suficiente provar que não é nula para $z \in] 0, \infty[$. Mais especificamente, vamos provar que $\frac{d^{2} K}{d z^{2}}(z)<0$ para $z$ positivo. Lembramos que

$$
\frac{d^{2} K}{d z^{2}}(z)=\frac{2-2 \exp (3 z)+\exp (z)\left(-6+z^{3}\right)+\exp (2 z)\left(6+z^{3}\right)}{z^{3}(\exp (z)-1)^{3}},
$$

e portanto basta provar que o numerador é negativo para $z>0$. Para isso definimos a função numerador como segue

$$
\operatorname{Num}_{0}(z) \stackrel{\text { def }}{=} 2-2 \exp (3 z)+\exp (z)\left(-6+z^{3}\right)+\exp (2 z)\left(6+z^{3}\right)
$$

para $z \geq 0$. A partir dela definimos as seguintes funções também para $z$ não negativo

$$
\begin{aligned}
& \operatorname{Num}_{1}(z) \stackrel{\text { def }}{=} \exp (-z) \frac{d N u m_{0}(z)}{d z}=-6-6 \exp (2 z)+3 z^{2}+z^{3}+\exp (z)\left(12+3 z^{2}+2 z^{3}\right), \\
& N_{4}(z) \stackrel{\text { def }}{=} \frac{d N u m_{1}(z)}{d z}=-12 \exp (2 z)+3 z(2+z)+\exp (z)\left(12+6 z+9 z^{2}+2 z^{3}\right), \\
& N_{u m}(z) \stackrel{\text { def }}{=} \frac{d N u m_{2}(z)}{d z}=-24 \exp (2 z)+6(1+z)+\exp (z)\left(18+24 z+15 z^{2}+2 z^{3}\right), \\
& \operatorname{Num}_{4}(z) \stackrel{\text { def }}{=} \frac{d N u m_{3}(z)}{d z}=6-48 \exp (2 z)+\exp (z)\left(42+54 z+21 z^{2}+2 z^{3}\right), \\
& \operatorname{Num}_{5}(z) \stackrel{\text { def }}{=} \exp (-z) \frac{d N u m_{4}(z)}{d z}=96+96 z+27 z^{2}+2 z^{3}-96 \exp (z) .
\end{aligned}
$$


Seja $z>0$, então temos que

$$
96 \exp (z)>96\left(1+z+\frac{z}{2}^{2}+\frac{z}{6}^{3}\right)>96+96 z+27 z^{2}+2 z^{3},
$$

quer dizer, $\mathrm{Num}_{5}$ é uma função negativa para $z$ positivo. Isto implica que a função $N u m_{4}$ é estritamente decrescente, obtendo assim que

$$
\operatorname{Num}_{4}(z)<N u m_{4}(0)=0 .
$$

Essa última relação, implica por sua vez que a função $N u m_{3}$ é estritamente decrescente. Aplicando o mesmo argumento obtemos que $\operatorname{Num}_{3}(z)<\operatorname{Num}_{3}(0)=0$. Continuando com o mesmo procedimento para $\operatorname{Num}_{2}(z), \quad \operatorname{Num}_{1}(z)$ e $\operatorname{Num}_{0}(z)$ chegamos à relação $\operatorname{Num}_{0}(z)<\operatorname{Num}_{0}(0)=0$ para $z>0$. Portanto obtuvimos o procurado, concluindo assim que a segunda derivada da função $K$ é negativa, em particular não nula, para $z>0$.

Corolário A.6. A derivada da função $K$ atinge seu máximo na origem, ou seja

$$
\frac{d K}{d z}(z) \leq \frac{1}{12}, \quad z \in \mathbb{R}
$$

Prova: Pelo lema anterior temos que a origem é o único ponto crítico de $K$. Pelo mesmo lema também concluímos que $K$ é crescente para $z<0$ e decrescente para $z>0$. Logo

$$
\frac{d K}{d z}(z) \leq\left.\frac{d K}{d z}(z)\right|_{z=0}=\frac{1}{12}
$$

Assim resulta provado o corolário. 


\section{Apêndice B}

\section{Demonstrações dos resultados do capítulo 2}

\section{Resultados da seção 2.1}

Prova da Proposição 2.1.2: Como a função exponencial é contínua, ela é uniformemente contínua num compacto. Logo, para cada $\epsilon>0$, obtemos que existe $\delta>0$ tal que

$$
|z-w|<\delta \Rightarrow|\exp (z)-\exp (w)|<\epsilon
$$

Por outro lado, dado que a função $h$ é contínua e positiva num compacto então a função logarítmo $\ln (h)$ está bem definida e também é contínua num compacto. Como conseqüência do Teorema de Stone-Weierstrass, ver Rudin (1976) ou Lages Lima (2003), temos que $\ln (h)$ pode ser aproximada uniformemente por polinômios nas variáveis $x$ e $y$. Isto implica que existe um polinômio $P_{n}(x, y)=\sum_{i, j=0}^{n} \lambda_{i j} x^{i} y^{j}$ tal que

$$
\left|\ln (h(x, y))-P_{n}(x, y)\right|<\delta \quad \text { para todo } \quad(x, y) \in[a, b] \times[a, b] .
$$

Portanto, juntando os dois resultados, obtemos que

$$
\left|h(x, y)-\exp \left(P_{n}(x, y)\right)\right|<\epsilon \quad \text { para todo } \quad(x, y) \in[a, b] \times[a, b] .
$$

Ou seja, a função $h$ pode ser uniformemente aproximada pela exponencial de polinômios nas variáveis $x$ e $y$. 
Prova do Lema 2.1.3: De acordo com a Proposição 2.1.2, existe uma seqüência $\left(\exp \left(P_{n}\right)\right)_{n \in \mathbb{N}}$ que aproxima uniformemente à densidade $h$. Para cada $n \in \mathbb{N}$, definimos a seqüência de constantes normalizadoras $\left(c_{n}\right)_{n \in \mathbb{N}}$ sendo

$$
c_{n}=\left(\int_{a}^{b} \int_{a}^{b} \exp \left(P_{n}(x, y)\right) d x d y\right)^{-1} .
$$

Dado que $h$ possui integral total 1 e a convergência é uniforme, concluímos que $c_{n} \stackrel{n \rightarrow+\infty}{\longrightarrow} 1$. Logo, a seqüência de densidades $\left(c_{n} \exp \left(P_{n}\right)\right)_{n \in \mathbb{N}}$ converge uniformemente para $h$.

\section{Resultados da seção 2.2}

A seguir enunciamos algumas propriedades que são necessárias para demonstrar os resultados apresentados em $\S 2.2$. Começamos enunciando o Teorema de Dini e o Teorema da Convergência Dominada. A demonstração do primeiro pode ser vista, por exemplo, em Rudin (1976), página 150, ou em Lages Lima (2003), página 211. Também em Rudin (1976), na página 321, é dada uma prova do Teorema da Convergência Dominada.

Teorema de Dini. Se uma seqüência de funções reais contínuas $\left(I_{n}\right)_{n \in \mathbb{N}}, I_{n}: S \mapsto \mathbb{R}$, definidas num espaço métrico compacto $S$, converge pontualmente para uma função contínua $I_{n}: S \mapsto \mathbb{R}$ $e$, além disso, tem-se $I_{1}(s) \leq I_{2}(s) \leq \cdots \leq I_{n}(s) \leq \cdots$ para todo $s \in S$ então a convergência $I_{n} \rightarrow I$ é uniforme em $S$.

Teorema da Convergência Dominanda. Seja $\left(I_{n}\right)_{n \in \mathbb{N}}$ uma seqüência de funções mensuráveis e $\mathcal{I}$ é uma função integrável. Se $I_{n} \rightarrow I$ em quase todo ponto e $\left|I_{n}\right| \leq \mathcal{I}$ em quase todo ponto para todo $n \in \mathbb{N}$, então $\lim _{n \rightarrow+\infty} \int I_{n}=\int I$.

Lema B.1. Sejam $S, b \in] 0,+\infty[$ e h uma função positiva e contínua em $[-S, S] \times[-b, b]$. A função

$$
\begin{aligned}
I & : \quad[-S, S] \mapsto \mathbb{R}^{+} \\
I(x) & =\int_{-b}^{b} h(x, y) d y
\end{aligned}
$$

é contínua. 
Prova: Seja $x_{0} \in[-S, S]$. Provaremos que para qualquer sequiência $\left(x_{n}\right)_{n \in \mathbb{N}} \subset[-S, S]$ que converge para $x_{0}$ obtemos que $I\left(x_{n}\right)$ tende para $I\left(x_{0}\right)$.

Pela continuidade de $h$ sabemos que $h\left(x_{n}, y\right) \stackrel{n \rightarrow+\infty}{\longrightarrow} h\left(x_{0}, y\right)$ para cada $y \in[-b, b]$. Também, pela continuidade de $h$, deve existir $\kappa \in] 0,+\infty[$ tal que

$$
\kappa=\max _{(x, y) \in[-S, S] \times[-b, b]} h(x, y) .
$$

Em particular $h\left(x_{n}, y\right) \leq \kappa$ para todo $n \in \mathbb{N}$ e, claramente, $\kappa$ possui integral finita em $[-S, S] \times[-b, b]$. Assim podemos aplicar o Teorema da Convergência Dominada e obter que

$$
\begin{aligned}
\lim _{n \rightarrow+\infty} \int_{-b}^{b} h\left(x_{n}, y\right) d y & =\int_{-b}^{b} h\left(x_{0}, y\right) d y \\
\lim _{n \rightarrow+\infty} I\left(x_{n}\right) & =I\left(x_{0}\right) .
\end{aligned}
$$

Concluímos que a função $I$ é contínua.

Corolário B.2. Sejam $S, b \in] 0,+\infty[$ e h uma função positiva e contínua em $[-S, S] \times[-b, b]$. A função

$$
\begin{aligned}
I & : \quad[-S, S] \mapsto \mathbb{R}^{+} \\
I(y) & =\int_{-b}^{b} h(x, y) d x
\end{aligned}
$$

é contínua.

Prova: A demonstração é conseqüência imediata do Lema B.1 já que a prova do lema não depende da variável de integração.

Prova do Lema 2.2.2: Começamos provando que para todo $n \in \mathbb{N}$

$$
\int_{-n}^{n} h(x, y) d y \leq f_{X}(x) .
$$

Dado que $h(x, y)>0$ para todo $(x, y) \in \mathbb{R} \times \mathbb{R}$, então

$$
\int_{-n}^{n} h(x, y) d y \leq \int_{-(n+j)}^{n+j} h(x, y) d y, \quad \text { para } j \in \mathbb{N} .
$$


Tomando o limite de $j$ tendendo para infinito obtemos

$$
\int_{-n}^{n} h(x, y) d y \leq \int_{-\infty}^{+\infty} h(x, y) d y=f_{X}(x) .
$$

Provamos a seguir o primeiro item do Lema. Como $h \in \mathcal{H}$, pela propriedade ii) da definição de $\mathcal{H}$, temos que para qualquer $\epsilon>0$, existe $S>0$ tal que se $|x|>S$ então $f_{X}(x)<\epsilon$.

Por outro lado, sabemos pela equação (B.1) e o Lema B.1, que $\int_{-n}^{n} h(x, y) d y$ define uma seqüência crescente de funções contínuas na variável $x$. Dado que tal seqüência converge pontualmente para $f_{X}$ se $|x| \leq S$, pelo Teorema de Dini, converge uniformemente em $x$ para $|x| \leq S$. Então

$$
\int_{-\infty}^{-n} h(x, y) d y+\int_{n}^{+\infty} h(x, y) d y
$$

converge uniformemente para zero quando $|x| \leq S$. Isto significa que existe $n_{0} \in \mathbb{N}$ tal que se $n \geq n_{0}$ então

$$
\int_{-\infty}^{-n} h(x, y) d y+\int_{n}^{+\infty} h(x, y) d y<\epsilon \quad \text { para todo } x \in[-S, S] .
$$

Agora consideramos $x$ tal que $|x|>S$ e $n \geq n_{0}$, obtemos que

$$
\int_{-\infty}^{-n} h(x, y) d y+\int_{n}^{+\infty} h(x, y) d y=f_{X}(x)-\int_{-n}^{n} h(x, y) d y<f_{X}(x)<\epsilon,
$$

a última desigualdade é conseqüência da definição de $S$. Portanto, fica provada a convergência uniforme em $x$ para $x \in \mathbb{R}$.

O segundo item é provado analogamente.

Prova da Proposição 2.2.3: Seja $\epsilon>0$. Provamos primeiramente a desigualdade envolvendo a densidade conjunta $h$.

Pela propriedade i) da definição de $\mathcal{H}$ existe $n_{1} \in \mathbb{N}$ tal que se $\|(x, y)\|>n_{1}$, então $h(x, y)<\frac{\epsilon}{2}$.

Seja $n \geq n_{1}$. Para $(x, y) \in[-n, n] \times[-n, n]$ obtemos

$$
\left|h(x, y)-h_{n}(x, y)\right|=|h(x, y)-h(x, y)|=0<\frac{\epsilon}{2} .
$$


Para $(x, y) \notin[-n, n] \times[-n, n]$ vale que $x^{2}>n^{2}$ ou $y^{2}>n^{2}$ então $x^{2}+y^{2}>n^{2}>n_{1}^{2}$, isto significa que $\|(x, y)\|>n_{1}$. Concluímos que

$$
\left|h(x, y)-h_{n}(x, y)\right|=h(x, y)<\frac{\epsilon}{2} .
$$

Portanto para $n \geq n_{1}$

$$
\sup _{(x, y) \in \mathbb{R} \times \mathbb{R}}\left|h(x, y)-h_{n}(x, y)\right| \leq \frac{\epsilon}{2}<\epsilon .
$$

A seguir trabalhamos com a desigualdade da densidade marginal $f_{X}$. Pelo primeiro item do Lema 2.2.2, sabemos que existe $n_{2} \in \mathbb{N}$ tal que se $n \geq n_{2}$, então

$$
\left|f_{X}(x)-\int_{-n}^{n} h(x, y) d y\right|=\left|\int_{-\infty}^{-n} h(x, y) d y+\int_{n}^{\infty} h(x, y) d y\right|<\frac{\epsilon}{2},
$$

para todo $x \in \mathbb{R}$. Por outro lado, pela propriedade ii) da definição de $\mathcal{H}$ existe $n_{3} \in \mathbb{N}$ tal que se $|x|>n_{3}$, então $f_{X}(x)<\frac{\epsilon}{2}$.

Definimos $n_{4}=\max \left\{n_{2}, n_{3}\right\}$ e consideramos $n \geq n_{4}$. Se $|x| \leq n$, obtemos

$$
\left|f_{X}(x)-f_{X}^{n}(x)\right|=\left|f_{X}(x)-\int_{-n}^{n} h(x, y) d y\right|<\frac{\epsilon}{2} .
$$

Se $|x|>n$, obtemos

$$
\left|f_{X}(x)-f_{X}^{n}(x)\right|=f_{X}(x)<\frac{\epsilon}{2} .
$$

Logo, para $n \geq n_{4}$

$$
\sup _{x \in \mathbb{R}}\left|f_{X}(x)-f_{X}^{n}(x)\right| \leq \frac{\epsilon}{2}<\epsilon .
$$

Para a marginal $g_{Y}$ procedemos analogamente obtendo um $n_{5} \in \mathbb{N}$ tal que para $n \geq n_{5}$ vale que

$$
\sup _{y \in \mathbb{R}}\left|g_{Y}(y)-g_{Y}^{n}(y)\right|<\epsilon .
$$

Finalmente, tomando $n_{0}=\max \left\{n_{1}, n_{4}, n_{5}\right\}$, a proposição resulta provada.

Prova do Teorema 2.2.6: Necessitamos provar que para todo $\epsilon>0$ existe $n_{0} \in \mathbb{N}$ tal que para $n \geq n_{0}$ obtemos

$$
\begin{aligned}
\sup _{(x, y) \in \mathbb{R} \times \mathbb{R}}\left|h(x, y)-\overline{\mathcal{E}}_{n}(x, y)\right| & <\epsilon, \\
\sup _{x \in \mathbb{R}}\left|f_{X}(x)-f_{X}^{\overline{\mathcal{E}}_{n}}(x)\right| & <\epsilon, \\
\sup _{y \in \mathbb{R}}\left|g_{Y}(y)-g_{Y}^{\overline{\mathcal{E}}_{n}}(y)\right| & <\epsilon .
\end{aligned}
$$


Seja $\epsilon>0$, pela Proposição 2.2.3 existe $n_{1} \in \mathbb{N}$ tal que para todo $n \geq n_{1}$ temos que

$$
\begin{aligned}
\sup _{(x, y) \in \mathbb{R} \times \mathbb{R}}\left|h(x, y)-h_{n}(x, y)\right| & <\frac{\epsilon}{4}, \\
\sup _{x \in \mathbb{R}} & \left|f_{X}(x)-f_{X}^{n}(x)\right|<\frac{\epsilon}{4}, \\
\sup _{y \in \mathbb{R}} & \left|g_{Y}(y)-g_{Y}^{n}(y)\right|<\frac{\epsilon}{4},
\end{aligned}
$$

sendo $\left(h_{n}\right)_{n \in \mathbb{N}},\left(f_{X}^{n}\right)_{n \in \mathbb{N}}$ e $\left(g_{Y}^{n}\right)_{n \in \mathbb{N}}$ a seqüência truncada de $h$.

Pela propriedade $i$ ) da definição de $\mathcal{H}$ existe $n_{2} \in \mathbb{N}$ tal que se $\|(x, y)\|>n_{2}$, então $h(x, y)<\frac{\epsilon}{2}$. Definimos $n_{3}=\max \left\{n_{1}, n_{2}, \frac{2}{\epsilon}\right\}$. Agora tomamos $n \geq n_{3}$, em particular obtemos $\frac{1}{n^{3}} \leq \frac{1}{n} \leq \frac{1}{n_{3}} \leq \frac{\epsilon}{2}$. Se $(x, y) \in[-n, n] \times[-n, n]$, então

$$
\left|h(x, y)-\overline{\mathcal{E}}_{n}(x, y)\right|=\left|h_{n}(x, y)-\overline{\mathcal{E}}_{n}(x, y)\right|<\frac{1}{n^{3}} \leq \frac{\epsilon}{2} .
$$

Observamos que o termo $1 / n^{3}$ é conseqüência da construção de $\overline{\mathcal{E}}_{n}$ no Lema 2.2.5.

Se $(x, y) \notin[-n, n] \times[-n, n]$, então $\|(x, y)\|>n \geq n_{3} \geq n_{2}$ e isto implica que

$$
\left|h(x, y)-\overline{\mathcal{E}}_{n}(x, y)\right|=h(x, y)<\frac{\epsilon}{2} .
$$

Portanto, para $n \geq n_{3}$ obtemos que $\sup _{(x, y) \in \mathbb{R} \times \mathbb{R}}\left|h(x, y)-\overline{\mathcal{E}}_{n}(x, y)\right|<\epsilon$.

Agora trabalhamos analogamente com a densidade marginal $f_{X}$. Pela propriedade ii) da definição de $\mathcal{H}$ existe $n_{4} \in \mathbb{N}$ tal que $|x|>n_{4}$ implica $f_{X}(x)<\frac{\epsilon}{2}$. Definimos $n_{5}=\max \left\{n_{4}, n_{1}, \frac{4}{\epsilon}\right\}$ e tomamos $n \geq n_{5}$, em particular $\frac{1}{n^{3}} \leq \frac{\epsilon}{4}$. Se $x \in[-n, n]$, então

$$
\left|f_{X}(x)-f_{X}^{\overline{\mathcal{E}}_{n}}(x)\right| \leq\left|f_{X}(x)-f_{X}^{n}(x)\right|+\left|f_{X}^{n}(x)-f_{X}^{\overline{\mathcal{E}}_{n}}(x)\right|<\frac{\epsilon}{4}+\frac{1}{n^{3}} \leq \frac{\epsilon}{2} .
$$

Se $x \notin[-n, n]$, então $|x|>n \geq n_{5} \geq n_{4}$ e isto implica que

$$
\left|f_{X}(x)-f_{X}^{\overline{\mathcal{E}}_{n}}(x)\right|=f_{X}(x)<\frac{\epsilon}{2} .
$$

Logo, para $n \geq n_{5}$ obtemos que $\sup _{x \in \mathbb{R}}\left|f_{X}(x)-f_{X}^{\overline{\mathcal{E}}_{n}}(x)\right|<\epsilon$. 
De forma análoga podemos definir $n_{6} \in \mathbb{N}$ tal que se $n \geq n_{6}$, obtemos que $\sup _{y \in \mathbb{R}}\left|g_{Y}(y)-g_{Y}^{\overline{\mathcal{E}}_{n}}(y)\right|<\epsilon$.

Finalmente, tomando $n_{0}=\max \left\{n_{3}, n_{5}, n_{6}\right\}$ as desigualdades (B.2), (B.3) e (B.4) resultam provadas.

Prova da Proposição 2.2.7: Provamos primeiro o item 1. Seja $a_{x}=-\infty$ e $b_{x}, a_{y}, b_{y} \in \mathbb{R}$. Tomamos $n \geq \max \left\{b_{x},-a_{y}, b_{y}\right\}$, então

$$
\begin{aligned}
\left|\mathbb{P}\left(a_{x}<X<b_{x}, a_{y}<Y<b_{y}\right)-\mathbb{P}_{\overline{\mathcal{E}}_{n}}\left(a_{x}<X<b_{x}, a_{y}<Y<b_{y}\right)\right| \\
\quad=\left|\int_{-\infty}^{b_{x}} \int_{a_{y}}^{b_{y}} h(x, y)-\overline{\mathcal{E}}_{n}(x, y) d y d x\right| \\
\leq\left|\int_{-\infty}^{-n} \int_{a_{y}}^{b_{y}} h(x, y) d y d x\right|+\left|\int_{-n}^{b_{x}} \int_{a_{y}}^{b_{y}} h(x, y)-\overline{\mathcal{E}}_{n}(x, y) d y d x\right| \\
\leq\left|\int_{-\infty}^{-n} \int_{-\infty}^{+\infty} h(x, y) d y d x\right|+\left|\int_{-n}^{b_{x}} \int_{a_{y}}^{b_{y}} h_{n}(x, y)-\overline{\mathcal{E}}_{n}(x, y) d y d x\right| \\
\leq \int_{-\infty}^{-n} f_{X}(x) d x+\frac{\left(b_{y}-a_{y}\right)\left(b_{x}+n\right)}{n^{3}} .
\end{aligned}
$$

Os termos acima convergem para zero quando $n \rightarrow+\infty$.

A seguir procedemos similarmente para provar o segundo item. Seja $a_{x}=a_{y}=-\infty \mathrm{e}$ $b_{x}, b_{y} \in \mathbb{R}$. Tomamos $n \geq \max \left\{b_{x}, b_{y}\right\}$, então 


$$
\begin{aligned}
&\left|\mathbb{P}\left(a_{x}<X<b_{x}, a_{y}<Y<b_{y}\right)-\mathbb{P}_{\overline{\mathcal{E}}_{n}}\left(a_{x}<X<b_{x}, a_{y}<Y<b_{y}\right)\right| \\
&=\left|\int_{-\infty}^{b_{x}} \int_{-\infty}^{b_{y}} h(x, y)-\overline{\mathcal{E}}_{n}(x, y) d y d x\right| \\
& \leq \mid\left|\int_{-\infty}^{-n} \int_{-\infty}^{b_{y}} h(x, y) d y d x\right|+\left|\int_{-n}^{b_{x}} \int_{-\infty}^{-n} h(x, y) d y d x\right| \\
&+\left|\int_{-n}^{b_{x}} \int_{-n}^{b_{y}} h(x, y)-\overline{\mathcal{E}}_{n}(x, y) d y d x\right| \\
& \leq\left|\int_{-\infty}^{-n} \int_{-\infty}^{+\infty} h(x, y) d y d x\right|+\left|\int_{-\infty}^{-n} \int_{-\infty}^{+\infty} h(x, y) d x d y\right| \\
&+\left|\int_{-n}^{b_{x}} \int_{-n}^{b_{y}} h_{n}(x, y)-\overline{\mathcal{E}}_{n}(x, y) d y d x\right| \\
& \leq \int_{-\infty}^{-n} f_{X}(x) d x+\int_{-\infty}^{-n} g_{Y}(y) d y+\frac{\left(b_{y}+n\right)\left(b_{x}+n\right)}{n^{3}} .
\end{aligned}
$$

Tomando o limite quando $n \rightarrow+\infty$, obtemos a convergência das probabilidades.

Finalmente estudamos o terceiro caso, $a_{x}=a_{y}=-\infty, b_{y}=+\infty$ e $b_{x} \in \mathbb{R}$. Consideramos $n \geq b_{x}$.

$$
\begin{aligned}
\left|\mathbb{P}\left(a_{x}<X<b_{x}, a_{y}<Y<b_{y}\right)-\mathbb{P}_{\overline{\mathcal{E}}_{n}}\left(a_{x}<X<b_{x}, a_{y}<Y<b_{y}\right)\right| \\
\quad=\left|\int_{-\infty}^{b_{x}} f_{X}(x)-f_{X}^{\overline{\mathcal{E}}_{n}}(x) d x\right| \\
\leq\left|\int_{-\infty}^{-n} f_{X}(x) d x\right|+\left|\int_{-n}^{b_{x}} f_{X}(x)-f_{X}^{\overline{\mathcal{E}}_{n}}(x) d x\right| \\
\leq\left|\int_{-\infty}^{-n} f_{X}(x) d x\right|+\left|\int_{-n}^{b_{x}} f_{X}(x)-f_{X}^{n}(x) d x\right|+\left|\int_{-n}^{b_{x}} f_{X}^{n}(x)-f_{X}^{\overline{\mathcal{E}}_{n}}(x) d x\right| \\
\leq \int_{-\infty}^{-n} f_{X}(x) d x+\int_{-n}^{b_{x}}\left(\int_{-\infty}^{+\infty} h(x, y) d y-\int_{-n}^{n} h(x, y) d y\right) d x+\frac{\left(b_{x}+n\right)}{n^{3}}
\end{aligned}
$$


Então

$$
\begin{aligned}
\mid \mathbb{P}\left(a_{x}\right. & \left.<X<b_{x}, a_{y}<Y<b_{y}\right)-\mathbb{P}_{\overline{\mathcal{E}}_{n}}\left(a_{x}<X<b_{x}, a_{y}<Y<b_{y}\right) \mid \\
& \leq \int_{-\infty}^{-n} f_{X}(x) d x+\int_{-n}^{b_{x}}\left(\int_{-\infty}^{-n} h(x, y) d y+\int_{n}^{+\infty} h(x, y) d y\right) d x+\frac{\left(b_{x}+n\right)}{n^{3}} \\
& \leq \int_{-\infty}^{-n} f_{X}(x) d x+\int_{-\infty}^{+\infty}\left(\int_{-\infty}^{-n} h(x, y) d y+\int_{n}^{+\infty} h(x, y) d y\right) d x+\frac{\left(b_{x}+n\right)}{n^{3}} \\
& =\int_{-\infty}^{-n} f_{X}(x) d x+\int_{-\infty}^{-n} g_{Y}(y) d y+\int_{n}^{+\infty} g(y) d y+\frac{\left(b_{x}+n\right)}{n^{3}} .
\end{aligned}
$$

Os quatros termos acima convergem para zero quando $n$ tende para infinito. Portanto a proposição resulta provada.

Prova da Proposição 2.2.9: Definimos

$$
R=\max \left\{r_{1}, r_{2}, \cdots, r_{M}\right\}
$$

e

$$
S=\max \left\{s_{1}, s_{2}, \cdots, s_{M}\right\}
$$

Construímos a seqüência $\left(\overline{\mathcal{E}}_{n}\right)_{n \in \mathbb{N}}$ como feito anteriormente no Lema 2.2.5, mas mudando as equações (2.1), (2.2) e (2.3) pelas seguintes

$$
\begin{aligned}
\sup _{(x, y) \in[-n, n] \times[-n, n]}\left|h_{n}(x, y)-\overline{\mathcal{E}}_{n}(x, y)\right| & <\frac{1}{n^{3+R+S}}, \\
\sup _{x \in[-n, n]}\left|f_{X}^{n}(x)-f_{X}^{\overline{\mathcal{E}}_{n}}(x)\right| & <\frac{1}{n^{3+R+S}}, \\
\sup _{y \in[-n, n]}\left|g_{Y}^{n}(y)-g_{Y}^{\overline{\mathcal{E}}_{n}}(y)\right| & <\frac{1}{n^{3+R+S}} .
\end{aligned}
$$

A convergência de $\left(\overline{\mathcal{E}}_{n}\right)_{n \in \mathbb{N}}$ e das correspondentes marginais pode ser mostrada similarmente ao feito na prova do Teorema 2.2.6. A seguir demonstramos a convergência dos momentos. Para 
qualquer $i \in\{1,2, \cdots, M\}$ temos que

$$
\begin{aligned}
\mid \mathbb{E} & {\left[X^{r_{i}} Y^{s_{i}}\right]-\mathbb{E}_{\overline{\mathcal{E}}_{n}}\left[X^{r_{i}} Y^{s_{i}}\right] \mid } \\
& =\left|\mathbb{E}\left[X^{r_{i}} Y^{s_{i}}\right]-\int_{-n}^{n} \int_{-n}^{n} x^{r_{i}} y^{s_{i}} \overline{\mathcal{E}}_{n}(x, y) d x d y\right| \\
& \leq\left|\mathbb{E}\left[X^{r_{i}} Y^{s_{i}}\right]-\int_{-n}^{n} \int_{-n}^{n} x^{r_{i}} y^{s_{i}} h(x, y) d x d y\right|+\left|\int_{-n}^{n} \int_{-n}^{n} x^{r_{i}} y^{s_{i}}\left(h(x, y)-\overline{\mathcal{E}}_{n}(x, y)\right) d x d y\right| \\
& =\left|\mathbb{E}\left[X^{r_{i}} Y^{s_{i}}\right]-\int_{-n}^{n} \int_{-n}^{n} x^{r_{i}} y^{s_{i}} h(x, y) d x d y\right|+\left|\int_{-n}^{n} \int_{-n}^{n} x^{r_{i}} y^{s_{i}}\left(h_{n}(x, y)-\overline{\mathcal{E}}_{n}(x, y)\right) d x d y\right| \\
& \leq\left|\mathbb{E}\left[X^{r_{i}} Y^{s_{i}}\right]-\int_{-n}^{n} \int_{-n}^{n} x^{r_{i}} y^{s_{i}} h(x, y) d x d y\right|+n^{r_{i}+s_{i}} \int_{-n}^{n} \int_{-n}^{n}\left|h_{n}(x, y)-\overline{\mathcal{E}}_{n}(x, y)\right| d x d y \\
& \leq\left|\mathbb{E}\left[X^{r_{i}} Y^{s_{i}}\right]-\int_{-n}^{n} \int_{-n}^{n} x^{r_{i}} y^{s_{i}} h(x, y) d x d y\right|+4 \frac{n^{2+r_{i}+s_{i}}}{n^{3+R+S}} \\
& \leq\left|\mathbb{E}\left[X^{r_{i}} Y^{s_{i}}\right]-\int_{-n}^{n} \int_{-n}^{n} x^{r_{i}} y^{s_{i}} h(x, y) d x d y\right|+\frac{4}{n} .
\end{aligned}
$$

Tomando o limite quando $n \rightarrow+\infty$ fica provada a proposição. 


\section{Apêndice $\mathrm{C}$}

\section{Demonstrações dos resultados do capítulo 3}

\section{Resultados da seção 3.2.1}

Proposição C.1. Seja $f_{X}$ definida por

$f_{X}(x)= \begin{cases}\exp \left(\lambda_{0}+\lambda_{10} x\right) \exp \left(\left(\lambda_{01}+\lambda_{11} x\right) a\right)\left(\frac{\exp \left(\left(\lambda_{01}+\lambda_{11} x\right)(b-a)\right)-1}{\lambda_{01}+\lambda_{11} x}\right), & \text { se } \lambda_{01}+\lambda_{11} x \neq 0 \\ \exp \left(\lambda_{0}+\lambda_{10} x\right)(b-a), & \text { se } \lambda_{01}+\lambda_{11} x=0 .\end{cases}$

Então

$$
\frac{d f_{X}}{d x}(x)=f_{X}(x)\left(\lambda_{11} \psi\left(\lambda_{01}+\lambda_{11} x\right)+\lambda_{10}+a \lambda_{11}\right),
$$

sendo

$$
\psi(z) \stackrel{\text { def }}{=} \begin{cases}\frac{(b-a) \exp ((b-a) z)}{\exp ((b-a) z)-1}-\frac{1}{z}, & \text { se } z \neq 0 \\ \frac{b-a}{2}, & \text { se } z=0\end{cases}
$$


Prova: A prova consiste em simplesmente derivar,

$$
\begin{aligned}
\frac{d f_{X}}{d x}(x)= & \lambda_{10} f_{X}(x)-\frac{\lambda_{11}}{\lambda_{01}+\lambda_{11} x} f_{X}(x) \\
& +a \lambda_{11} f_{X}(x)+(b-a) \lambda_{11} \exp \left(\lambda_{0}+\lambda_{10} x+\left(\lambda_{01}+\lambda_{11} x\right) a\right) \frac{\exp \left(\left(\lambda_{01}+\lambda_{11} x\right)(b-a)\right)}{\lambda_{01}+\lambda_{11} x} \\
= & f_{X}(x)\left(\lambda_{10}+a \lambda_{11}+(b-a) \lambda_{11}+\frac{(b-a) \lambda_{11}}{\exp \left(\left(\lambda_{01}+\lambda_{11} x\right)(b-a)\right)-1}-\frac{\lambda_{11}}{\lambda_{01}+\lambda_{11} x}\right) \\
= & f_{X}(x)\left(\lambda_{10}+a \lambda_{11}+\frac{(b-a) \lambda_{11} \exp \left(\left(\lambda_{01}+\lambda_{11} x\right)(b-a)\right)}{\exp \left(\left(\lambda_{01}+\lambda_{11} x\right)(b-a)\right)-1}-\frac{\lambda_{11}}{\lambda_{01}+\lambda_{11} x}\right) \\
= & f_{X}(x)\left(\lambda_{10}+a \lambda_{11}+\lambda_{11} \psi\left(\lambda_{01}+\lambda_{11} x\right)\right) .
\end{aligned}
$$

Prova da Proposição 3.2.2: A prova da continuidade e dos limitantes é conseqüência imediata da definição de $L\left(z, \lambda ; \lambda_{11}\right)$ e das propriedades de $\psi$ enunciadas na Proposição 3.2.1. Para analisar o comportamento monotônico calculamos cada derivada parcial.

No caso da variavel $z$ obtemos que

$$
\frac{\partial L}{\partial z}\left(z, \lambda ; \lambda_{11}\right)=-\left.\left(\lambda_{11}\right)^{2} \frac{d \psi}{d w}(w)\right|_{w=\lambda+z \lambda_{11}} .
$$

Como a função $\psi$ é estritamente crescente, a derivada é estritamente positiva e portanto $\frac{\partial L}{\partial z}\left(z, \lambda ; \lambda_{11}\right)$ é negativa, ou seja, $L\left(z, \lambda ; \lambda_{11}\right)$ é decrescente na variavel $z$.

Estudemos agora a variável $\lambda$.

$$
\frac{\partial L}{\partial \lambda}\left(z, \lambda ; \lambda_{11}\right)=-\left.\lambda_{11} \frac{d \psi}{d w}(w)\right|_{w=\lambda+z \lambda_{11}} .
$$

A expressão acima é positiva para valores negativos de $\lambda_{11}$ e negativa caso contrário. Isto implica que $L\left(z, \lambda ; \lambda_{11}\right)$ é crescente na variável $\lambda$ quando $\lambda_{11}<0$ e decrescente quando $\lambda_{11}>0$.

Prova da Proposição 3.2.3: A prova da convexidade consiste em ver que as segundas derivadas são funções positivas para quiasquer que sejam os valores de $\lambda_{10}, \lambda_{01}$ e $\lambda_{11}$. Começamos calculando a segunda derivada da função $f_{X}$. 


$$
\begin{aligned}
\frac{d^{2} f_{X}}{d x^{2}}(x) & =\frac{d}{d x}\left[f_{X}(x)\left(\lambda_{11} \psi\left(\lambda_{01}+\lambda_{11} x\right)+\lambda_{10}+a \lambda_{11}\right)\right] \\
& =\frac{d f_{X}}{d x}(x)\left(\lambda_{11} \psi\left(\lambda_{01}+\lambda_{11} x\right)+\lambda_{10}+a \lambda_{11}\right)+\lambda_{11}^{2} f_{X}(x) \frac{d \psi}{d x}\left(\lambda_{01}+\lambda_{11} x\right) \\
& =f_{X}(x)\left(\lambda_{11} \psi\left(\lambda_{01}+\lambda_{11} x\right)+\lambda_{10}+a \lambda_{11}\right)^{2}+\left.\lambda_{11}^{2} f_{X}(x) \frac{d \psi}{d w}(w)\right|_{w=\lambda_{01}+\lambda_{11} x}
\end{aligned}
$$

A expressão acima é positiva pois $\lambda_{11} \neq 0$ e a função $\psi$ possui primeira derivada positiva.

A prova para a marginal $g_{Y}$ é similar já que a segunda derivada está dada por

$$
\frac{d^{2} g_{Y}}{d y^{2}}(y)=g_{Y}(y)\left[\left(\lambda_{11} \psi\left(\lambda_{10}+\lambda_{11} y\right)+\lambda_{01}+a \lambda_{11}\right)^{2}+\left.\lambda_{11}^{2} \frac{d \psi}{d w}(w)\right|_{w=\lambda_{10}+\lambda_{11} y}\right] \text {. }
$$

Concluímos assim que ambas marginais são estritamente convexas.

Prova da Proposição 3.2.4: Analisamos cada item.

1. Seja $\lambda_{11}>0$.

(a) Consideremos $-a \lambda_{11} \leq \lambda_{10}$. Pela Proposição 3.2 .2 sabemos que $L\left(a, \lambda_{01} ; \lambda_{11}\right)<-a \lambda_{11}$. Então, juntando ambas desigualdades, obtemos $L\left(a, \lambda_{01} ; \lambda_{11}\right)<\lambda_{10}$, ou seja, $f_{X}$ é uma função crescente segundo a Tabela 3.2.1.

(b) Se $-b \lambda_{11} \geq \lambda_{10}$, usamos a relação $L\left(b, \lambda_{01} ; \lambda_{11}\right)>-b \lambda_{11}$ para obter $L\left(b, \lambda_{01} ; \lambda_{11}\right)>\lambda_{10}$ e assim concluir que $f_{X}$ é decrescente, ver Tabela 3.2.1.

Os itens (c) e (d) podem ser provados de forma análoga substituindo $\lambda_{10}$ por $\lambda_{01}$ e viceversa.

2. Seja $\lambda_{11}<0$. Neste caso a demonstração de cada item é similar a dos casos anteriores mas usando as desigualdades correspondentes da Proposição 3.2.2, isto é $-a \lambda_{11}<L\left(z, \lambda ; \lambda_{11}\right)<-b \lambda_{11}$.

Prova da Proposição 3.2.5: Analisamos cada item.

1. Se $\left.x_{0} \in\right] a, b\left[\right.$ é um minimizador de $f_{X}(x)$ então

$$
\left.\frac{d f_{X}(x)}{d x}\right|_{x=x_{0}}=0
$$

ou, equivalentemente,

$$
f_{X}(x)\left[\lambda_{11} \psi\left(\lambda_{01}+x_{0} \lambda_{11}\right)+\lambda_{10}+a \lambda_{11}\right]=0 .
$$


Isto implica que

$$
L\left(x_{0}, \lambda_{01} ; \lambda_{11}\right)=\lambda_{10} .
$$

Reciprocamente, se a igualdade acima for satisfeita, então a derivada de $f_{X}$ em $x_{0}$ é nula. Como $f_{X}$ é estritamente convexa, $x_{0}$ é um minimizador.

2. A prova para a densidade marginal $g_{Y}$ é similar, simplesmente substituindo $\lambda_{10}$ por $\lambda_{01}$ e $\lambda_{01}$ por $\lambda_{10}$.

Prova da Proposição 3.2.6: Analisamos cada item.

1. A prova do primeiro item é imediata, pois

$$
L\left(a, \lambda+(b-a) \lambda_{11} ; \lambda_{11}\right)=-\lambda_{11}\left(\psi\left(\lambda+(b-a) \lambda_{11}+a \lambda_{11}\right)+a\right)=-\lambda_{11}\left(\psi\left(\lambda+b \lambda_{11}\right)+a\right) .
$$

O último termo é por definição $L\left(b, \lambda ; \lambda_{11}\right)$.

2. Provamos a seguir o segundo item. Começamos com a existência de $\lambda_{a}^{0}$. Consideramos primeiro o caso $\lambda_{11}>0$. Pelos limitantes dados na Proposição 3.2.2 temos que

$$
L\left(a,-a \lambda_{11} ; \lambda_{11}\right)<-a \lambda_{11} \quad \text { e } \quad L\left(a,-b \lambda_{11} ; \lambda_{11}\right)>-b \lambda_{11} .
$$

Como a função $L\left(a, \lambda ; \lambda_{11}\right)-\lambda$ é contínua em $\lambda$, podemos afirmar, pelo Teorema do valor intermediário, que existe um $\lambda_{a}^{0}$ tal que $-b \lambda_{11}<\lambda_{a}^{0}<-a \lambda_{11}$ e $L\left(a, \lambda_{a}^{0} ; \lambda_{11}\right)-\lambda_{a}^{0}=0$, ou seja $L\left(a, \lambda_{a}^{0} ; \lambda_{11}\right)=\lambda_{a}^{0}$.

Neste caso em que $\lambda_{11}>0$ a unicidade é trivial pois a função $L\left(a, \lambda ; \lambda_{11}\right)$ é estritamente decrescente em $\lambda$ e portanto pode cortar uma única vez a reta $r(\lambda)=\lambda$, que é crescente.

Para $\lambda_{11}<0$ a demonstração da existência é análoga ao caso $\lambda_{11}>0$ mas utilizando as desigualdades

$$
L\left(a,-a \lambda_{11} ; \lambda_{11}\right)>-a \lambda_{11} \quad \text { e } \quad L\left(a,-b \lambda_{11} ; \lambda_{11}\right)<-b \lambda_{11} .
$$

Para provar a unicidade precisamos conhecer as seguintes derivadas

$$
\frac{\partial L}{\partial \lambda}\left(a, \lambda ; \lambda_{11}\right)=-\left.\lambda_{11} \frac{d \psi}{d w}(w)\right|_{w=a \lambda_{11}+\lambda}=-\left.\lambda_{11}(b-a)^{2} \frac{d K}{d w}(w)\right|_{w=(b-a)\left(a \lambda_{11}+\lambda\right)}
$$

e

$$
\frac{\partial^{2} L}{\partial \lambda^{2}}\left(a, \lambda ; \lambda_{11}\right)=-\left.\lambda_{11} \frac{d^{2} \psi}{d w^{2}}(w)\right|_{w=a \lambda_{11}+\lambda}=-\left.\lambda_{11}(b-a)^{3} \frac{d^{2} K}{d w^{2}}(w)\right|_{w=(b-a)\left(a \lambda_{11}+\lambda\right)} .
$$


Pelo Lema A.5 ambas derivadas são contínuas. Raciocinando pelo absurdo, assumimos que existem pelo menos dois pontos $\lambda_{a}^{0}<\tilde{\lambda}_{a}^{0}$ tais que $L\left(a, \lambda_{a}^{0} ; \lambda_{11}\right)-\lambda_{a}^{0}=0=L\left(a, \tilde{\lambda}_{a}^{0} ; \lambda_{11}\right)-\tilde{\lambda}_{a}^{0}$. Pela continuidade na variável $\lambda$ da função $L\left(a, \lambda ; \lambda_{11}\right)$ e pelas desigualdades

$$
-a \lambda_{11}<L\left(a, \lambda ; \lambda_{11}\right)<-b \lambda_{11}
$$

somente podemos ter as duas situações esquematizadas na Figura C.1.1. Em qualquer uma delas deve existir um ponto $\alpha$ tal que $-a \lambda_{11}<\alpha<-b \lambda_{11}$ e no qual a função $L\left(a, \lambda ; \lambda_{11}\right)$ muda de concavidade, ou seja, $\left.\frac{\partial^{2} L}{\partial \lambda^{2}}\left(a, \lambda ; \lambda_{11}\right)\right|_{\lambda=\alpha}=0$. Isto é conseqüência do Teorema do valor intermediário e da continuidade da primeira e da segunda derivada em $\lambda$ da função $L$. Da expressão da segunda derivada $\frac{\partial^{2} L}{\partial \lambda^{2}}$ e do Lema A.5 concluímos que $\alpha=-a \lambda_{11}$, o qual é um absurdo já que deve ser estritamente maior do que $-a \lambda_{11}$. Portanto existe um único $\lambda_{a}^{0}$ tal que $L\left(a, \lambda_{a}^{0} ; \lambda_{11}\right)=\lambda_{a}^{0}$.

Figura C.1.1

Situações possíveis no caso de não unicidade de $\lambda_{a}^{0}$ para $\lambda_{11}<0$.

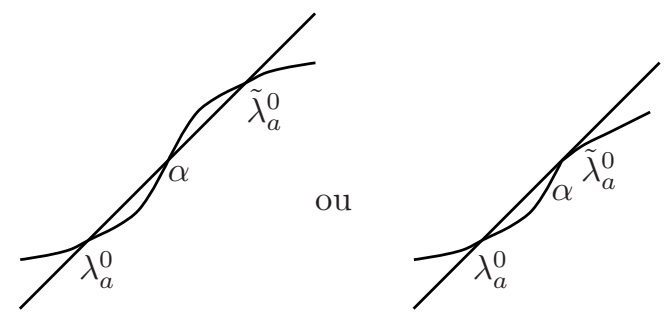

A prova da existência e unicidade de $\lambda_{b}^{0}$ pode ser realizada aplicando o mesmo procedimento para a função $L\left(b, \lambda ; \lambda_{11}\right)-\lambda$.

3. Demonstramos agora o terceiro item. Vamos provar um problema equivalente que é a existência e unicidade de um $\lambda$ tal que $L\left(b, L\left(a, \lambda ; \lambda_{11}\right) ; \lambda_{11}\right)=\lambda$. Usamos o mesmo argumento do item anterior. Assumimos $\lambda_{11}>0$, então

$$
L\left(b, L\left(a,-a \lambda_{11} ; \lambda_{11}\right) ; \lambda_{11}\right)<-a \lambda_{11} \quad \text { e } \quad L\left(b, L\left(a,-b \lambda_{11} ; \lambda_{11}\right) ; \lambda_{11}\right)>-b \lambda_{11} .
$$

Logo, pela continuidade da função $L\left(b, L\left(a, \lambda ; \lambda_{11}\right) ; \lambda_{11}\right)-\lambda$ temos que existe $\lambda$ tal que $-b \lambda_{11}<\lambda<-a \lambda_{11}$ e $L\left(b, L\left(a, \lambda ; \lambda_{11}\right) ; \lambda_{11}\right)=\lambda$. Definimos $\lambda_{b}^{*}=\lambda$ e $\lambda_{a}^{*}=L\left(a, \lambda_{b}^{*} ; \lambda_{11}\right)$. 
Assim fica provada a existência de tais valores para $\lambda_{11}>0$. Para o caso $\lambda_{11}<0$ procedemos da mesma maneira mas usando as desigualdades

$$
L\left(b, L\left(a,-a \lambda_{11} ; \lambda_{11}\right) ; \lambda_{11}\right)>-a \lambda_{11} \quad \text { e } \quad L\left(b, L\left(a,-b \lambda_{11} ; \lambda_{11}\right) ; \lambda_{11}\right)<-b \lambda_{11} .
$$

A prova da unicidade é feita como no item anterior. Assumimos que existe mais de um ponto $\lambda$ satisfazendo $L\left(b, L\left(a, \lambda ; \lambda_{11}\right) ; \lambda_{11}\right)=\lambda$ e obtemos uma contradição. Novamente a contradição é dada pela existência de um ponto diferente da origem que anula a segunda derivada da função $K$. Neste caso a segunda derivada está dada por

$$
\frac{\partial^{2}}{\partial \lambda^{2}} L\left(b, L\left(a, \lambda ; \lambda_{11}\right) ; \lambda_{11}\right)=\left(-\lambda_{11}\right)^{2}(b-a)^{5}(A+B)
$$

sendo

$$
A=-\left.\lambda_{11}(b-a)^{2}\left(\left.\frac{d K}{d w}(w)\right|_{w=(b-a)\left(a \lambda_{11}+\lambda\right)}\right)^{2} \frac{d^{2} K}{d w^{2}}(w)\right|_{w=(b-a)\left(b \lambda_{11}+L\left(a, \lambda ; \lambda_{11}\right)\right.}
$$

e

$$
B=\left.\left.\frac{d K}{d w}(w)\right|_{w=(b-a)\left(b \lambda_{11}+L\left(a, \lambda ; \lambda_{11}\right)\right.} \frac{d^{2} K}{d w^{2}}(w)\right|_{w=(b-a)\left(a \lambda_{11}+\lambda\right)} .
$$

Se $\lambda_{11}>0$, então $w=(b-a)\left(b \lambda_{11}+L\left(a, \lambda ; \lambda_{11}\right)\right)>0$ e isto implica que $\frac{d^{2} K}{d w^{2}}(w)<0$ (ver prova do Lema A.5). Logo o termo $A$ é positivo. O termo $B$ também é positivo para $\lambda_{11}>0$, pois a primeira derivada da função $K$ é sempre positiva pela Proposição A.1 e a segunda derivada é positiva para $a \lambda_{11}+\lambda<0$ (ver prova do Lema A.5). Logo, para que a segunda derivada em $\lambda$ da função $L$, dada na equação (C.2), seja nula, os dois termos $A$ e $B$ devem ser nulos. Isto implica que deve ser

$$
\left.\frac{d^{2} K}{d w^{2}}(w)\right|_{w=(b-a)\left(b \lambda_{11}+L\left(a, \lambda ; \lambda_{11}\right)\right)}=0 \quad \text { e }\left.\quad \frac{d^{2} K}{d w^{2}}(w)\right|_{w=(b-a)\left(a \lambda_{11}+\lambda\right)}=0 .
$$

Novamente, pelo Lema A.5 concluímos que $\lambda=-a \lambda_{11}$ e $L\left(a, \lambda ; \lambda_{11}\right)=-b \lambda_{11}$. Ambas igualdades são absurdas e portanto concluímos que existe um único $\lambda$ tal que $L\left(b, L\left(a, \lambda ; \lambda_{11}\right) ; \lambda_{11}\right)=\lambda$. Isto implica imediatamente a unicidade de $\lambda_{a}^{*}$ e $\lambda_{b}^{*}$.

Agora somente falta provar a unicidade para o caso em que $\lambda_{11}$ é negativo. Neste caso precisamos de um raciocínio diferente. Começamos definindo a função

$$
\begin{aligned}
\left.L^{-1}\left(b, \cdot ; \lambda_{11}\right): \quad\right]-a \lambda_{11},-b \lambda_{11}[\mapsto & \mathbb{R} \\
L^{-1}\left(b, \lambda ; \lambda_{11}\right)=\beta & \leftrightarrow\left(b, \beta ; \lambda_{11}\right)=\lambda .
\end{aligned}
$$


Sabemos que existem $\lambda_{a}^{*}$ e $\lambda_{b}^{*}$ tais que $-a \lambda_{11}<\lambda_{b}^{*}<-b \lambda_{11},-a \lambda_{11}<\lambda_{a}^{*}<-b \lambda_{11}, L\left(b, \lambda_{a}^{*} ; \lambda_{11}\right)=$ $\lambda_{b}^{*}$ e $L\left(a, \lambda_{b}^{*} ; \lambda_{11}\right)=\lambda_{a}^{*}$, ou seja, $L\left(a, \lambda_{b}^{*} ; \lambda_{11}\right)=L^{-1}\left(b, \lambda_{b}^{*} ; \lambda_{11}\right)$. Provaremos que $\lambda_{b}^{*}$ é o único $\lambda$ que satisfaz a igualdade $L\left(a, \lambda ; \lambda_{11}\right)=L^{-1}\left(b, \lambda ; \lambda_{11}\right)$.

Se $\lambda$ é tal que $L\left(a, \lambda ; \lambda_{11}\right)=L^{-1}\left(b, \lambda ; \lambda_{11}\right)$, então $\lambda<L\left(b,-b \lambda_{11} ; \lambda_{11}\right)$. Logo, é suficiente provar a unicidade no intervalo $]-a \lambda_{11}, L\left(b,-b \lambda_{11} ; \lambda_{11}\right)[$. Por outro lado, temos que $0<\lambda_{a}^{*}-\lambda_{b}^{*}<L\left(b,-b \lambda_{11} ; \lambda_{11}\right)+a \lambda_{11}=L\left(a,-a \lambda_{11} ; \lambda_{11}\right)+a \lambda_{11}$. A positividade anterior é provada no Corolário 3.2.8, (salientamos que na prova dessa desigualdade do corolário não é utilizada a presente proposição).

Seja $m(\lambda)=\lambda+\lambda_{a}^{*}-\lambda_{b}^{*}$. Se $m(\lambda)$ corta em mais de um ponto a curva $L\left(a, \lambda ; \lambda_{11}\right)$ para $\lambda>-a \lambda_{11}$, então deve ser $-a \lambda_{11}+\lambda_{a}^{*}-\lambda_{b}^{*} \geq L\left(a-a \lambda_{11} ; \lambda_{11}\right)$. Isto implica que $\lambda_{a}^{*}-\lambda_{b}^{*} \geq$ $L\left(a,-a \lambda_{11} ; \lambda_{11}\right)+a \lambda_{11}$, o qual é um absurdo. Portanto a reta $m(\lambda)$ corta a curva $L\left(a, \lambda ; \lambda_{11}\right)$ unicamente em $\lambda=\lambda_{b}^{*}$. Obtemos assim que

$$
\begin{aligned}
& m(\lambda)<L\left(a, \lambda ; \lambda_{11}\right), \text { se }-a \lambda_{11}<\lambda<\lambda_{b}^{*} \\
& m(\lambda)>L\left(a, \lambda ; \lambda_{11}\right), \text { se }-a \lambda_{11}<\lambda<\lambda_{b}^{*} .
\end{aligned}
$$

Por outro lado, se $m(\lambda)$ corta duas vezes a curva $L^{-1}\left(b, \lambda ; \lambda_{11}\right)$ para $-a \lambda_{11}<\lambda<L\left(b,-b \lambda_{11} ; \lambda_{11}\right)$, então $m\left(L\left(b,-b \lambda_{11}, \lambda_{11}\right) \geq L^{-1}\left(b, L\left(b,-b \lambda_{11} ; \lambda_{11}\right) ; \lambda_{11}\right)\right.$. Assim obtemos que $-b \lambda_{11}=$ $2 L\left(b,-b \lambda_{11} ; \lambda_{11}\right)+a \lambda_{11}>-b \lambda_{11}$, o qual é um absurdo, portanto a reta $m(\lambda)$ corta a curva $L^{-1}\left(b, \lambda ; \lambda_{11}\right)$ unicamente em $\lambda=\lambda_{b}^{*}$. Obtemos assim que

$$
\begin{aligned}
& m(\lambda)>L^{-1}\left(b, \lambda ; \lambda_{11}\right), \text { se }-a \lambda_{11}<\lambda<\lambda_{b}^{*} \\
& m(\lambda)<L^{-1}\left(b, \lambda ; \lambda_{11}\right) \text {, se }-a \lambda_{11}<\lambda<\lambda_{b}^{*} .
\end{aligned}
$$

Das desigualdades (C.3), (C.4), (C.5) e (C.6), concluímos que $L\left(a, \lambda ; \lambda_{11}\right)$ e $L^{-1}\left(b, \lambda ; \lambda_{11}\right)$ se cortam unicamente em $\lambda=\lambda_{b}^{*}$.

Prova do Corolário 3.2.8: Começamos provando as desigualdades para o caso $\lambda_{11}>0$. Lembramos que nesse caso a função $L\left(z, \lambda ; \lambda_{11}\right)$ é decrescente na variável $z$ e na variavél $\lambda$. Como

$$
\begin{aligned}
L\left(b, L\left(a,-b \lambda_{11} ; \lambda_{11}\right) ; \lambda_{11}\right)-\left(-b \lambda_{11}\right) & >-b \lambda_{11}-\left(-b \lambda_{11}\right)=0, \\
L\left(b, L\left(a, \lambda_{b}^{0} ; \lambda_{11}\right) ; \lambda_{11}\right)-\lambda_{b}^{0} & <L\left(b, L\left(b, \lambda_{b}^{0} ; \lambda_{11}\right) ; \lambda_{11}\right)-\lambda_{b}^{0}=0
\end{aligned}
$$


e a função $L$ é contínua, existe um $\lambda$ tal que $-b \lambda_{11}<\lambda<\lambda_{b}^{0}$ e $L\left(b, L\left(a, \lambda ; \lambda_{11}\right) ; \lambda_{11}\right)=\lambda$. Pela prova da Proposição 3.2.6 temos que $\lambda=\lambda_{b}^{*}$, $\log 0-b \lambda_{11}<\lambda_{b}^{*}<\lambda_{b}^{0}$.

Provemos a seguir que $\lambda_{b}^{0}<\lambda_{a}^{0}$, para isso assumimos, raciocinando pelo absurdo, que $\lambda_{b}^{0} \geq \lambda_{a}^{0}$ então temos que

$$
\lambda_{b}^{0}=L\left(b, \lambda_{b}^{0} ; \lambda_{11}\right)<L\left(a, \lambda_{b}^{0} ; \lambda_{11}\right) \leq L\left(a, \lambda_{a}^{0} ; \lambda_{11}\right)=\lambda_{a}^{0} .
$$

Logo temos uma contradição com o nosso suposto de $\lambda_{b}^{0} \geq \lambda_{a}^{0}$, portanto deve ser $\lambda_{b}^{0}<\lambda_{a}^{0}$. Finalizamos este caso provando que $\lambda_{a}^{0}<\lambda_{a}^{*}<-a \lambda_{11}$. Temos que

$$
\begin{aligned}
L\left(a, L\left(b, \lambda_{a}^{0} ; \lambda_{11}\right) ; \lambda_{11}\right)-\lambda_{a}^{0} & >L\left(a, L\left(a, \lambda_{a}^{0} ; \lambda_{11}\right) ; \lambda_{11}\right)-\lambda_{a}^{0}=0 \\
L\left(a, L\left(b,-a \lambda_{11} ; \lambda_{11}\right) ; \lambda_{11}\right)-\left(-a \lambda_{11}\right) & <-a \lambda_{11}-\left(-a \lambda_{11}\right)=0 .
\end{aligned}
$$

Novamente pela continuidade da função $L$, deve existir $\lambda$ tal que $\lambda_{a}^{0}<\lambda<-a \lambda_{11}$ e $L\left(a, L\left(b, \lambda ; \lambda_{11}\right) ; \lambda_{11}\right)=\lambda$. Pela prova da Proposição 3.2 .6 temos que $\lambda=\lambda_{a}^{*}$, logo $\lambda_{a}^{0}<\lambda_{a}^{*}<-a \lambda_{11}$.

Agora vamos provar as correspondentes desigualdades para $\lambda_{11}<0$. Neste caso a função $L\left(z, \lambda ; \lambda_{11}\right)$ é decrescente na variável $z$ e crescente na variável $\lambda$. Por outro lado, como consequiência da prova da Proposição 3.2.6, temos a seguinte relação

$$
\lambda<\lambda_{b}^{*}<\alpha \Rightarrow L\left(b, L\left(a, \lambda ; \lambda_{11}\right) ; \lambda_{11}\right)>\lambda \quad \text { e } \quad L\left(b, L\left(a, \alpha ; \lambda_{11}\right) ; \lambda_{11}\right)<\alpha .
$$

Começamos provando que $\lambda_{b}^{0}<\lambda_{b}^{*}$, realizamos isto pelo absurdo, supondo que $\lambda_{b}^{0} \geq \lambda_{b}^{*}$, pela relação (C.7) temos que

$$
L\left(b, L\left(a, \lambda_{b}^{0} ; \lambda_{11}\right) ; \lambda_{11}\right) \leq \lambda_{b}^{0}=L\left(b, \lambda_{b}^{0} ; \lambda_{11}\right) .
$$

Por ser $L$ crescente na variável $\lambda$ obtemos que

$$
L\left(a, \lambda_{b}^{0} ; \lambda_{11}\right) \leq \lambda_{b}^{0}=L\left(b, \lambda_{b}^{0} ; \lambda_{11}\right) .
$$

Esta última relação contradiz o fato da função $L\left(z, \lambda ; \lambda_{11}\right)$ ser decrescente na variável $z$. Logo deve ser $\lambda_{b}^{0}<\lambda_{b}^{*}$. Da mesma forma, trabalhando com a função $L\left(a, L\left(b, \lambda_{a}^{*} ; \lambda_{11}\right) ; \lambda_{11}\right)$, provamos que $\lambda_{a}^{*}<\lambda_{a}^{0}$. 
Agora somente falta ver que $\lambda_{b}^{*}<\lambda_{a}^{*}$. Novamente trabalhamos assumindo o contrário, ou seja, $\lambda_{b}^{*} \geq \lambda_{a}^{*}$ então

$$
\begin{array}{r}
L\left(a, \lambda_{b}^{*} ; \lambda_{11}\right) \geq L\left(a, \lambda_{a}^{*} ; \lambda_{11}\right)>L\left(b, \lambda_{a}^{*} ; \lambda_{11}\right) \\
\lambda_{a}^{*} \geq L\left(a, \lambda_{a}^{*} ; \lambda_{11}\right)>\lambda_{b}^{*} .
\end{array}
$$

Ou seja, partimos de $\lambda_{b}^{*} \geq \lambda_{a}^{*}$ e obtemos $\lambda_{b}^{*}<\lambda_{a}^{*}$. A contradição obtida prova que $\lambda_{b}^{*}<\lambda_{a}^{*}$.

\section{Resultados da seção 3.2.2}

Prova da Proposição 3.2.9: Começamos aplicando a regra de Leibniz obtendo assim que

$$
\frac{d f_{X}}{d x}(x)=\int_{a}^{b} h(x, y)\left(\lambda_{10}+\lambda_{11} y+2 \lambda_{20} x\right) d y=\int_{a}^{b} h(x, y) P_{f}(x, y) d y .
$$

Observemos primeiramente que o minimizador é um dos vértices do retângulo $[a, b] \times[a, b]$.

Começamos assumindo que $\min _{(x, y) \in[a, b] \times[a, b]} P_{f}(x, y) \geq 0$ e $\lambda_{11} \neq 0$. Então, para cada $\left.x \in\right] a, b[$ existe $\delta_{x}>0$ tal que $P_{f}(x, y) \geq \delta_{x}$ para $y \in[a, b]$. Por outro lado, como $h(x, y)$ é positiva podemos afirmar que

$$
\int_{a}^{b} h(x, y) P_{f}(x, y) d y \geq \delta_{x}>0
$$

ou seja, a derivada de $f_{X}$ é positiva para todo $\left.x \in\right] a, b[$.

Agora analisamos o caso $\lambda_{11}=0$, ou seja $P_{f}(x, y)=\lambda_{10}+2 \lambda_{20} x$. Neste caso é imediato ver que a derivada de $f_{X}$ é positiva para todo $\left.x \in\right] a, b[$.

O segundo item é provado com um raciocínio análogo, obtendo que a derivada de $f_{X}$ é negativa, $\log f_{X}$ é decrescente. 
Prova do Corolário 3.2.10: Cada item deste corolário mostra o minimizador de $P_{f}$ segundo a positividade dos coeficientes $\lambda_{20}$ e $\lambda_{11}$. Para $x$ fixo, $P_{f}$ define uma reta em $y$ de inclinação $\lambda_{11}$. Por outro lado, se fixarmos $y, P_{f}$ define uma reta em $x$ de inclinação $2 \lambda_{20}$. Com estas observações, e o fato do minimizador de $P_{f}$ ser um dos pontos $\{(a, a),(a, b),(b, b),(b, a)\}$, é imediato concluir que

1. $\lambda_{20}>0$ e $\lambda_{11} \geq 0$ implicam que $\min _{(x, y) \in[a, b] \times[a, b]} P_{f}(x, y)=P_{f}(a, a)$;

2. $\lambda_{20}>0$ e $\lambda_{11}<0$ implicam que $\min _{(x, y) \in[a, b] \times[a, b]} P_{f}(x, y)=P_{f}(a, b)$;

3. $\lambda_{20}<0$ e $\lambda_{11} \geq 0$ implicam que $\min _{(x, y) \in[a, b] \times[a, b]} P_{f}(x, y)=P_{f}(b, a)$;

4. $\lambda_{20}<0$ e $\lambda_{11}<0$ implicam que $\min _{(x, y) \in[a, b] \times[a, b]} P_{f}(x, y)=P_{f}(b, b)$.

Concluímos a demonstração aplicando a Proposição 3.2.9.

Prova da Proposição 3.2.12: Para provar que $x_{0}$ é um ponto crítico temos que ver que anula a derivada de $f_{X}$. Como $\lambda_{11}=0$ tal derivada resulta ser

$$
\left.\frac{d f_{X}}{d x}(x)\right|_{x=x_{0}}=\left.f_{X}(x)\left(\lambda_{10}+2 \lambda_{20} x\right)\right|_{x=-\frac{\lambda_{10}}{2 \lambda_{20}}}=0 .
$$

Assim concluímos que $x_{0}$ é um ponto crítico de $f_{X}$. A unicidade é imediata da expressão da derivada.

Prova da Proposição 3.2.13: Pela Proposição 3.2.12 sabemos que $x_{0}=-\frac{\lambda_{10}}{2 \lambda_{20}}$ é o único ponto crítico de $f_{X}$. Logo, para ver que $x_{0}$ é moda é suficiente provar que a segunda derivada de $f_{X}$ em $x_{0}$ é negativa. Sendo $\lambda_{11}=0$ obtemos que

$$
\left.\frac{d^{2} f_{X}}{d x^{2}}(x)\right|_{x=x_{0}}=f_{X}\left(x_{0}\right)\left(\left(\lambda_{10}-2 \lambda_{20} \frac{\lambda_{10}}{2 \lambda_{20}}\right)^{2}+2 \lambda_{20}\right)=2 \lambda_{20} .
$$

Como $\lambda_{20}<0$, concluímos que $\left.\frac{d^{2} f_{X}}{d x^{2}}(x)\right|_{x=x_{0}}$ e, portanto, $x_{0}$ é um maximizador de $f_{X}$. 
Prova da Proposição 3.2.14: Quando $\lambda_{11}=0$ a densidade marginal $f_{X}$ está dada por

$$
f_{X}(x)= \begin{cases}\exp \left(\lambda_{0}+\lambda_{10} x+\lambda_{20} x^{2}\right)\left(\frac{\exp \left(\lambda_{01} b\right)-\exp \left(\lambda_{01} a\right)}{\lambda_{01}}\right), & \text { se } \lambda_{01} \neq 0 \\ \exp \left(\lambda_{0}+\lambda_{10} x+\lambda_{20} x^{2}\right) \frac{(b-a)}{2}, & \text { se } \lambda_{01}=0\end{cases}
$$

Por outro lado, temos que o vértice da parábola $\lambda_{0}+\lambda_{10} x+\lambda_{20} x^{2}$ é o ponto médio do intervalo $[a, b]$, logo é imediato ver que $f_{X}$ é simétrica, ou seja,

$$
f_{X}\left(\frac{b+a}{2}+x\right)=f_{X}\left(\frac{b+a}{2}-x\right)
$$

para todo $x \in\left[0, \frac{b-a}{2}\right]$.

Prova do Lema 3.2.15: Começamos a demonstração calculando a derivada de $f_{X}$.

$$
\begin{aligned}
\frac{d f_{X}}{d x}(x)= & f_{X}(x)\left(\lambda_{10}+2 \lambda_{20} x+a \lambda_{11}+\lambda_{11}(b-a) \frac{\exp \left((b-a)\left(\lambda_{01}+\lambda_{11} x\right)\right)}{\exp \left((b-a)\left(\lambda_{01}+\lambda_{11} x\right)\right)-1}\right. \\
& \left.-\frac{\lambda_{11}}{\lambda_{01}+\lambda_{11} x}\right) \\
= & f_{X}(x)\left(\lambda_{10}+2 \lambda_{20} x+a \lambda_{11}+\lambda_{11} \psi\left(\lambda_{01}+\lambda_{11} x\right)\right) \\
= & f_{X}(x)\left(\lambda_{10}+2 \lambda_{20} x-L\left(x, \lambda_{01} ; \lambda_{11}\right)\right) .
\end{aligned}
$$

Logo a segunda derivada é

$$
\begin{aligned}
\frac{d^{2} f_{X}}{d x^{2}}(x) & =f_{X}(x)\left(\left(\lambda_{10}+2 \lambda_{20} x-L\left(x, \lambda_{01} ; \lambda_{11}\right)\right)^{2}+2 \lambda_{20}-\frac{\partial L}{\partial x}\left(x, \lambda_{01} ; \lambda_{11}\right)\right) \\
& =f_{X}(x)\left(\left(\lambda_{10}+2 \lambda_{20} x-L\left(x, \lambda_{01} ; \lambda_{11}\right)\right)^{2}+2 \lambda_{20}+\left.\lambda_{11}^{2} \frac{d \psi}{d w}\right|_{w=\lambda_{01}+\lambda_{11} x}\right) .
\end{aligned}
$$

Como $\lambda_{20}>0$ e $\psi$ possui derivada positiva, concluímos que $f_{X}$ é convexa. 
Prova da Proposição 3.2.16: Na demonstração do Lema 3.2 .15 vimos que

$$
\frac{d f_{X}}{d x}(x)=f_{X}(x)\left(\lambda_{10}+2 \lambda_{20} x-L\left(x, \lambda_{01} ; \lambda_{11}\right)\right) .
$$

Logo, $f_{X}$ possui derivada positiva se, e somente se, $\lambda_{10}+2 \lambda_{20} x-L\left(x, \lambda_{01} ; \lambda_{11}\right)>0$ para todo $x \in] a, b\left[\right.$. E $f_{X}$ possui derivada negativa se, e somente se, $\lambda_{10}+2 \lambda_{20} x-L\left(x, \lambda_{01} ; \lambda_{11}\right)<0$ para todo $x \in] a, b\left[\right.$. Como $\lambda_{20}>0$ e $L$ é decrescente na variável $x$ concluímos que

$$
f_{X}^{i} \Leftrightarrow \lambda_{10} \geq-2 \lambda_{20} a+L\left(a, \lambda_{01} ; \lambda_{11}\right)
$$

$\mathrm{e}$

$$
f_{X}^{d} \Leftrightarrow \lambda_{10} \leq-2 \lambda_{20} b+L\left(b, \lambda_{01} ; \lambda_{11}\right)
$$

Quando $-2 \lambda_{20} b+L\left(b, \lambda_{01} ; \lambda_{11}\right)<\lambda_{10}<\lambda_{20} a+L\left(a, \lambda_{01} ; \lambda_{11}\right)$, dado que a derivada de $f_{X}$ é contínua, obtemos que existe $x \in] a, b[$ com derivada nula, ou seja, com ponto crítico. Esse ponto é o único ponto crítico e é um minimizador pelo Lema 3.2.15. Assim fica provada a proposição.

Prova do Corolário 3.2.18: Começamos provando o primeiro item, assumindo que $\lambda_{11}>0$, $\lambda_{01}+\lambda_{11} a>0$ e $-2 \lambda_{20}-\lambda_{11}\left(\frac{b+a}{2}\right) \leq \lambda_{10}$. Como $\psi$ é crescente obtemos que

$$
\begin{aligned}
\psi\left(\lambda_{01}+\lambda_{11} a\right) & >\psi(0)=\frac{b-a}{2} \\
-\lambda_{11} \psi\left(\lambda_{01}+\lambda_{11} a\right)-\lambda_{11} a & <-\lambda_{11} \frac{b+a}{2} \\
-2 \lambda_{20} a+L\left(a, \lambda_{01} ; \lambda_{11}\right) & <-\lambda_{11} \frac{b+a}{2}-2 \lambda_{20} .
\end{aligned}
$$

Pela hipóteses, o termo da direita da expressão acima é menor ou igual do que $\lambda_{10} \mathrm{e}$, pela Proposição 3.2.16, isto implica que $f_{X}$ é crescente. De forma análoga podem ser provados os outros casos. 
Prova da Proposição 3.2.19: Provamos unicamente o caso $\lambda_{11}>0$, pois a demonstração para quando $\lambda_{11}<0$ é simétrica.

Primeiro assumimos que $a \geq \frac{-\lambda_{11} b-\lambda_{10}}{2 \lambda_{20}}$, ou seja $2 \lambda_{20} a+\lambda_{10} \leq-\lambda_{11} b$. Por outro lado $-\lambda_{11} b<L\left(x, \lambda_{01} ; \lambda_{11}\right)$. Assim obtemos que para $x>a$ valem as seguintes desigualdades

$$
\begin{aligned}
\frac{d f_{X}}{d x}(x) & =f_{X}(x)\left(\lambda_{10}+2 \lambda_{20} x-L\left(x, \lambda_{01} ; \lambda_{11}\right)\right) \\
& <f_{X}(x)\left(\lambda_{10}+2 \lambda_{20} a-L\left(x, \lambda_{01} ; \lambda_{11}\right)\right) \\
& \leq f_{X}(x)\left(-\lambda_{11} b-L\left(x, \lambda_{01} ; \lambda_{11}\right)\right) \\
& <0
\end{aligned}
$$

para $x \in] a, b\left[\right.$. Portanto $f_{X}$ é decrescente.

De forma análoga, mas utilizando a relação $-\lambda_{11} a>L\left(x, \lambda_{01} ; \lambda_{11}\right)$, prova-se o ítem 1.(c).

Finalizamos com a demonstração do caso $a<\frac{-\lambda_{11} a-\lambda_{10}}{2 \lambda_{20}}<\frac{-\lambda_{11} b-\lambda_{10}}{2 \lambda_{20}}<b$. Ou seja, $2 \lambda_{20} a+\lambda_{10}>-\lambda_{11} a>-\lambda_{11} b>2 \lambda_{20} b+\lambda_{10}$. Então obtemos que

$$
\lambda_{10}+2 \lambda_{20} b-L\left(b, \lambda_{01} ; \lambda_{11}\right)<0<\lambda_{10}+2 \lambda_{20} a-L\left(a, \lambda_{01} ; \lambda_{11}\right) .
$$

Pela continuidade da função $L\left(\cdot, \lambda_{01} ; \lambda_{11}\right)$ concluímos que existe $\left.x_{0} \in\right] a, b[$ tal que

$$
\lambda_{10}+2 \lambda_{20} x_{0}-L\left(x_{0}, \lambda_{01} ; \lambda_{11}\right)=0 .
$$

E portanto $\left.\frac{d f_{X}}{d x}(x)\right|_{x=x_{0}}=0$, ou seja, $x_{0}$ é um ponto crítico de $f_{X}$. Com isto resulta provada a proposição.

Prova da Proposição 3.2.20: A primeira derivada da função $f_{X}$ está dada por

$$
\frac{d f_{X}}{d x}(x)=f_{X}(x)\left(\lambda_{10}+2 \lambda_{20} x-L\left(x, \lambda_{01} ; \lambda_{11}\right)\right) .
$$

A seguir analisamos a derivada de $\mathcal{C}(x)=\lambda_{10}+2 \lambda_{20} x-L\left(x, \lambda_{01} ; \lambda_{11}\right)$

$$
\begin{aligned}
\frac{d \mathcal{C}}{d x}(x) & =2 \lambda_{20}-\frac{\partial L}{\partial x}\left(x, \lambda_{01} ; \lambda_{11}\right) \\
& =2 \lambda_{20}+\left.\lambda_{11}^{2} \frac{d \psi}{d w}\right|_{w=\lambda_{01}+\lambda_{11} x} \\
& =2 \lambda_{20}+\left.\lambda_{11}^{2}(b-a)^{2} \frac{d K}{d w}\right|_{w=(b-a)\left(\lambda_{01}+\lambda_{11} x\right)} .
\end{aligned}
$$


Aplicando o Corolário A.6, obtemos que

$$
-\left.\lambda_{11}^{2}(b-a)^{2} \frac{d K}{d w}\right|_{w=(b-a)\left(\lambda_{01}+\lambda_{11} x\right)} \geq-\lambda_{11}^{2} \frac{(b-a)^{2}}{12} .
$$

Por hipóteses temos que $\lambda_{20}<-\lambda_{11}^{2} \frac{(b-a)^{2}}{24}$, assim podemos concluir que a função $\mathcal{C}$ é decrescente. Isto implica que

$$
\left.f_{X}(x)\left(\lambda_{10}+2 \lambda_{20} b-L\left(b, \lambda_{01} ; \lambda_{11}\right)\right)<\frac{d f_{X}}{d x}(x)<f_{X}(x)\left(\lambda_{10}+2 \lambda_{20} a-L\left(a, \lambda_{01} ; \lambda_{11}\right)\right), \quad x \in\right] a, b[.
$$

Os dois primeiros itens da proposição são consequência inmediata das desigualdades acima. Para concluir o terceiro item observamos que, por ser $\mathcal{C}$ decrescente, $f_{X}$ possui no máximo um ponto crítico. Nesse ponto crítico a segunda derivada resulta ser negativa, ver equação (C.8). Portanto, nas hipóteses do terceiro item, existe um ponto crítico de $f_{X}$ em $] a, b[$ e é um maximizador.

Prova da Proposição 3.2.21: Aplicamos a regra de Leibniz e obtemos

$$
\frac{d g_{Y}}{d y}(y)=\int_{a}^{b} h(x, y)\left(\lambda_{01}+\lambda_{11} x\right) d x=\int_{a}^{b} h(x, y) P_{g}(x, 0) d x .
$$

Se $P_{g}(x, 0)>0$ para todo $x \in[a, b]$ então a expressão acima é positiva. Se $\min _{x \in[a, b]} P_{g}(x, 0)=0$ então $P_{g}(a, 0)=0$ ou $P_{g}(b, 0)=0$, segundo o signal de $\lambda_{11}$. Em qualquer um dos casos temos que

$$
\begin{aligned}
& \int_{a}^{b} h(x, y) P_{g}(x, 0) d x= \\
& \quad \int_{a}^{\frac{b+a}{4}} h(x, y) P_{g}(x, 0) d x+\int_{\frac{b+a}{4}}^{\frac{3(b+a)}{4}} h(x, y) P_{g}(x, 0) d x+\int_{\frac{3(b+a)}{4}}^{b} h(x, y) P_{g}(x, 0) d x .
\end{aligned}
$$

Como o segundo termo é positivo é o primeiro e terceiro são não negativos, concluimos que $\frac{d g_{Y}}{d y}(y)>0$, ou seja, $g_{Y}$ é crescente.

Analogamente provamos o caso decrescente. 
Prova da Proposição 3.2.24: Para ver que a função é convexa basta provar que a segunda derivada é não negativa, no caso de convexidade estrita, a segunda derivada deve ser positiva. Neste caso tal derivada é dada por

$$
\frac{d^{2} g_{Y}}{d y^{2}}=\int_{a}^{b} h(x, y)\left(\lambda_{01}+\lambda_{11} x\right)^{2} d x
$$

Logo, é imediata a prova da proposição.

\section{Resultados da seção 3.2.3}

Prova do Corolário 3.2.29: Dado que $\lambda_{20}=\lambda_{02}$ temos as seguintes relações

$$
\begin{aligned}
\min _{(x, y) \in[a, b] \times[a, b]}\left(P_{g}(x, y)-\lambda_{01}\right) & =\min _{(x, y) \in[a, b] \times[a, b]}\left(\lambda_{11} x+\lambda_{02} y\right) \\
& =\min _{(x, y) \in[a, b] \times[a, b]}\left(\lambda_{11} y+\lambda_{20} x\right) \\
& =\min _{(x, y) \in[a, b] \times[a, b]}\left(P_{f}(x, y)-\lambda_{10}\right)
\end{aligned}
$$

e, analogamente,

$$
\max _{(x, y) \in[a, b] \times[a, b]}\left(P_{g}(x, y)-\lambda_{01}\right)=\max _{(x, y) \in[a, b] \times[a, b]}\left(P_{f}(x, y)-\lambda_{10}\right) .
$$

Assim podemos afirmar que $\lambda_{10} \geq \lambda_{01}$ implica que $\min _{(x, y) \in[a, b] \times[a, b]} P_{g}(x, y) \leq \min _{(x, y) \in[a, b] \times[a, b]} P_{f}(x, y)$. Como $P_{g}$ é não negativo em $[a, b] \times[a, b]$ obtemos que $P_{f}$ também é. Aplicando as Proposições 3.2 .9 e 3.2 .26 resulta provado o primeiro item.

Os três itens restantes podem ser provados de forma similar.

Prova da Proposição 3.2.30: Começamos provando que $f_{X}$ é simétrica, neste caso isso é $f_{X}(x)=f_{X}(-x)$. Pela definição da densidade e a nulidade dos coeficientes $\lambda_{10}$ e $\lambda_{01}$ obtemos 
que

$$
\begin{aligned}
f_{X}(-x) & =\exp \left(\lambda_{0}+\lambda_{20}(-x)^{2}\right) \int_{-b}^{b} \exp \left(\lambda_{11}(-x) y+\lambda_{02} y^{2}\right) d y \\
& =\exp \left(\lambda_{0}+\lambda_{20} x^{2}\right)\left(-\int_{b}^{-b} \exp \left(\lambda_{11}(-x)(-y)+\lambda_{02}(-y)^{2}\right) d y\right) \\
& =\exp \left(\lambda_{0}+\lambda_{20} x^{2}\right) \int_{-b}^{b} \exp \left(\lambda_{11} x y+\lambda_{02} y^{2}\right) d y \\
& =f_{X}(x) .
\end{aligned}
$$

Assim resulta provado que $f_{X}(x)=f_{X}(-x)$.

A demonstração da simetría de $g_{Y}$ é análoga.

Prova da Proposição 3.2.31: Provamos unicamente o primeiro item pois o segundo consiste em simplesmente trocar $\lambda_{20}$ por $\lambda_{02}$.

A simetría das duas densidades é conseqüência da Proposição 3.2.30. Por sua vez, o fato de ser $f_{X}$ simétrica e não constante em $[a, b]=[-b, b]$, implica que $x_{0}=0$ é um ponto crítico de $f_{X}$. Vamos provar agora que $x_{0}$ é um maximizador demonstrando que $\left.\frac{d^{2} f_{X}}{d x^{2}}(x)\right|_{x=x_{0}}<0$. Mais ainda, provaremos que a derivada segunda é negativa para todo $x \in] a, b[$. Isto, por sua vez, implica que $x_{0}=0$ é o único maximizador, pois se existir outro então deve existir um minimizador e portanto um ponto em $] a, b[$ com segunda derivada positiva. Tal derivada está dada pela seguinte expressão

$$
\frac{d^{2} f_{X}}{d x^{2}}(x)=\int_{a}^{b} h(x, y)\left(\left(2 \lambda_{20} x+\lambda_{11} y\right)^{2}+2 \lambda_{20}\right) d y
$$

Como

$$
\max _{(x, y) \in[a, b] \times[a, b]}\left(\left(2 \lambda_{20} x+\lambda_{11} y\right)^{2}+2 \lambda_{20}\right)=2 \lambda_{20}+\left(-2 \lambda_{20} b+\operatorname{sgn}\left(\lambda_{11}\right) \lambda_{11} b\right)^{2}<0,
$$

obtemos que a derivada segunda é negativa em $] a, b[$. 


\section{Resultados da seção 3.3}

Prova do Corolário 3.3.2: Pretendemos mostrar que em cada um dos itens estamos dando o valor mínimo do polinômio $P_{f}(x, y)$. Primeiro observemos que o minimizador está num dos vértices do retângulo $[a, b] \times[a, b]$ pois a matriz hessiana

$$
\left(\begin{array}{ll}
\frac{\partial^{2} P_{f}}{\partial x^{2}} & \frac{\partial^{2} P_{f}}{\partial x \partial y} \\
\frac{\partial^{2} P_{f}}{\partial y \partial x} & \frac{\partial^{2} P_{f}}{\partial y^{2}}
\end{array}\right)=2 \lambda_{21}\left(\begin{array}{ll}
0 & 1 \\
1 & 0
\end{array}\right)
$$

é indefinida e $P_{f}(x, a), P_{f}(x, b), P_{f}(a, y)$ e $P_{f}(b, y)$ são retas.

A seguir calculamos o polinômio $P_{f}(x, y)$ nas diagonais de $[a, b] \times[a, b]$.

$$
\begin{aligned}
& y=x \quad \rightarrow P_{f}(x, x)=\lambda_{10}+\lambda_{11} x+2 \lambda_{21} x^{2}, \\
& y=b+a-x \rightarrow P_{f}(x, b+a-x)=\lambda_{10}+\lambda_{11}(a+b)+\left(-\lambda_{11}+2 \lambda_{21}(a+b)\right) x-2 \lambda_{21} x^{2} .
\end{aligned}
$$

Obtendo em ambos casos parábolas. A parábola $P_{f}(x, x)$ tem como vértice $x_{1}$ e a parábola $P_{f}(x, a+b-x)$ possui como vértice $x_{2}$. Por outro lado, se $\lambda_{21}>0$, então $P_{f}(x, x)$ é uma parábola convexa e $P_{f}(x, a+b-x)$ é uma parábola côncava. Como ambas parábolas possuem um ponto em comum, $\left(\frac{a+b}{2}, \frac{a+b}{2}\right)$, o minimizador de $P_{f}(x, y)$ é $(a, b)$ ou $(b, a)$. Simetricamente, se $\lambda_{21}<0$, então $P_{f}(x, x)$ é uma parábola côncava e $P_{f}(x, a+b-x)$ é uma parábola convexa, consequentemente o minimizador de $P_{f}(x, y)$ é $(a, a)$ ou $(b, b)$. Logo, pelas características conhecidas das parábolas, podemos afirmar que

1. se $\lambda_{21}>0, x_{2} \leq \frac{a+b}{2}$ então $\min _{(x, y) \in[a, b] \times[a, b]} P_{f}(x, y)=P_{f}(b, a)$;

2. se $\lambda_{21}>0, x_{2}>\frac{a+b}{2}$ então $\min _{(x, y) \in[a, b] \times[a, b]} P_{f}(x, y)=P_{f}(a, b)$;

3. se $\lambda_{21}<0, x_{1} \leq \frac{a+b}{2}$ então $\min _{(x, y) \in[a, b] \times[a, b]} P_{f}(x, y)=P_{f}(b, b)$;

4. se $\lambda_{21}<0, x_{1}>\frac{a+b}{2}$ então $\min _{(x, y) \in[a, b] \times[a, b]} P_{f}(x, y)=P_{f}(a, a)$.

Finalmente aplicamos a Proposição 3.3.1, e obtemos as condições do corolário.

Prova do Corolário 3.3.3: Demonstração análoga a prova do corolário anterior, neste caso procuramos sempre o vértice que é maximizador das parábolas definidas por $P_{f}(x, x)$ e $P_{f}(x, a+b-x)$. 
Prova da Proposição 3.3.4: Como $\lambda_{10}=0$ temos que

$$
f_{X}(x)= \begin{cases}\frac{\exp \left(\lambda_{0}\right)\left(\exp \left(\left(\lambda_{01}+\lambda_{11} x+\lambda_{21} x^{2}\right) b\right)-\exp \left(\left(\lambda_{01}+\lambda_{11} x+\lambda_{21} x^{2}\right) a\right)\right)}{\lambda_{01}+\lambda_{11} x+\lambda_{21} x^{2}}, & \text { se } \lambda_{01}+\lambda_{11} x+\lambda_{21} x^{2} \neq 0 \\ \exp \left(\lambda_{0}\right)(b-a), & \text { se } \lambda_{01}+\lambda_{11} x+\lambda_{21} x^{2}=0\end{cases}
$$

Por outro lado, como o ponto médio do intervalo $[a, b]$ é o vértice da parábola definida por $\lambda_{01}+\lambda_{11} x+\lambda_{21} x^{2}$ temos que

$$
\lambda_{01}+\lambda_{11}\left(\frac{a+b}{2}+x\right)+\lambda_{21}\left(\frac{a+b}{2}+x\right)^{2}=\lambda_{01}+\lambda_{11}\left(\frac{a+b}{2}-x\right)+\lambda_{21}\left(\frac{a+b}{2}-x\right)^{2},
$$

para qualquer $x \in\left[0, \frac{b-a}{2}\right]$. Com isso concluímos que $f_{X}$ é simétrica em $[a, b]$.

Prova da Proposição 3.3.5: Para ver que $x_{0}$ é ponto crítico de $f_{X}$ provamos que a derivada de $f_{X}$ é nula em $x_{0}$.

Se $\lambda_{10}=0$ e $\left.x_{0}=\frac{-\lambda_{11}}{2 \lambda_{21}} \in\right] a, b[$, então

$$
\begin{aligned}
\left.\frac{d f_{X}(x)}{d x}\right|_{x=x_{0}} & =\int_{a}^{b} h\left(x_{0}, y\right) P_{f}\left(x_{0}, y\right) d y \\
& =\int_{a}^{b} h\left(x_{0}, y\right)\left(\lambda_{11}+2 \lambda_{21} x_{0}\right) y d y=0 .
\end{aligned}
$$

Logo, $x_{0}$ é um ponto crítico de $f_{X}$.

Para provar a unicidade no caso $a \geq 0$ calculamos explicitamente a primeira derivada de $f_{X}$. Obtemos

$$
\frac{d f_{X}(x)}{d x}=f_{X}(x)\left(\lambda_{11}+2 \lambda_{21} x\right)\left(\psi\left(\lambda_{01}+\lambda_{11} x+\lambda_{21} x^{2}\right)+a\right),
$$

sendo $\psi$ a função definida na equação (3.3).

Precisamos provar que $\frac{d f_{X}}{d x}(x) \neq 0$ para todo $x \neq x_{0}$. Para isto é suficiente ver que $\psi\left(\lambda_{01}+\lambda_{11} x+\lambda_{21} x^{2}\right)+a>0$. Pela Proposição 3.2.1 temos que $\psi$ é sempre uma função positiva, por ser $a$ não negativo podemos concluir que $\psi\left(\lambda_{01}+\lambda_{11} x+\lambda_{21} x^{2}\right)+a>0$. Logo a derivada de $f_{X}$ só é nula em $x=x_{0}$. 
Prova da Proposição 3.3.6: Pela Proposição 3.3.5 sabemos que $f_{X}$ possui um único ponto crítico em $x_{0}$, somente falta provar que é a moda, ou seja, que $x_{0}$ é um maximizador. Isto é feito mostrando que a derivada segunda de $f_{X}$ no ponto $x=x_{0}$ é negativa.

$$
\begin{aligned}
\left.\frac{d^{2} f_{X}}{d x^{2}}(x)\right|_{x=x_{0}}= & f_{X}(x)\left[\left(\lambda_{11}+2 \lambda_{21} x\right)^{2}\left(\psi\left(\lambda_{01}+\lambda_{11} x+\lambda_{21} x^{2}\right)+a\right)^{2}\right. \\
& +2 \lambda_{21}\left(\psi\left(\lambda_{01}+\lambda_{11} x+\lambda_{21} x^{2}\right)+a\right) \\
& \left.+\left(\lambda_{11}+2 \lambda_{21} x\right)\left(\left.\left(\lambda_{11}+2 \lambda_{21} x\right) \frac{d \psi}{d w}(w)\right|_{w=\lambda_{01}+\lambda_{11} x+\lambda_{21} x^{2}}\right)\right]\left.\right|_{x=x_{0}} \\
= & 2 f_{X}(x) \lambda_{21}\left(\psi\left(\lambda_{01}+\lambda_{11} x+\lambda_{21} x^{2}\right)+a\right) \\
< & 0,
\end{aligned}
$$

a última desigualdade é consequiência de serem $\lambda_{21}<0, a \geq 0$ e $\psi$ uma função positiva.

Prova da Proposição 3.3.7: Precisamos mostrar que a derivada segunda de $f_{X}$ é não negativa para todo $x \in] a, b[$. Com tal fim, aplicamos duas vezes a regra de Leibniz obtendo assim que

$$
\frac{d^{2} f_{X}}{d x^{2}}(x)=\int_{a}^{b} h(x, y)\left(P_{f}(x, y)\right)^{2} d y+2 \lambda_{21} \int_{a}^{b} y h(x, y) d y .
$$

Tanto a hipótese $\lambda_{21}>0$ e $a \geq 0$ quanto a hipótese $\lambda_{21}<0$ e $b \leq 0$ implicam que a segunda derivada é não negativa.

Prova do Corolário 3.3.9: Começamos provando os três primeiros itens, ou seja $\lambda_{21}>0$, neste caso $P_{g}(x, 0)$ é uma parábola convexa. Logo, caso o vértice esteja no intervalo $[a, b]$, ele é o minimizador isto é

$$
\lambda_{21}>0, \quad a \leq-\frac{\lambda_{11}}{2 \lambda_{21}} \leq b \quad \Rightarrow \min _{x \in[a, b]} P_{g}(x, 0)=P_{g}\left(\frac{-\lambda_{11}}{2 \lambda_{21}}, 0\right) .
$$

Como $P_{g}\left(-\frac{\lambda_{11}}{2 \lambda_{21}}, 0\right) \geq 0$, pela Proposição 3.3 .8 fica provado o primeiro item. Se o vértice da parábola não estiver no intervalo $[a, b]$ então consideramos as duas possíveis situações. Quando o vértice estiver à esquerda do extremo $a$, ou seja $\frac{-\lambda_{11}}{2 \lambda_{21}}<a$, o mínimo de $P_{g}(x, 0)$ é $P_{g}(a, 0)$; quando o vértice estiver à direita do extremo $b$, ou seja $\frac{-\lambda_{11}}{2 \lambda_{21}}>b$, o mínimo de $P_{g}(x, 0)$ é $P_{g}(b, 0)$. Portanto, usando a Proposição 3.3.8 fica provado o item segundo e o terceiro. 
Agora analisamos o caso $\lambda_{21}<0$. Aqui a função $P_{g}(x, 0)$ é uma parábola côncava e portanto o minimizador é um dos extremos do domínio $[a, b]$. Se o vértice estiver mais perto do extremo inferior, ou seja $\frac{-\lambda_{11}}{2 \lambda_{21}} \leq \frac{a+b}{2}$, então o mínimo de $P_{g}(x, 0)$ é $P_{g}(b, 0)$, caso contrário o mínimo é $P_{g}(a, 0)$. Finalizamos a prova do quarto e quinto item utilizando novamente a Proposição 3.3.8.

Prova do Corolário 3.3.10: A demonstração é análoga à prova do corolário anterior, mas neste caso procuramos o maximizador de $P_{g}$.

Prova da Proposição 3.3.11: Aplicando duas vezes a regra de Leibniz obtemos $\frac{d^{2} g_{Y}}{d y^{2}}=\int_{a}^{b} h(x, y)\left(\lambda_{01}+\lambda_{11} x+\lambda_{21} x^{2}\right)^{2} d x$. Logo, a deriva segunda de $g_{Y}$ é não negativa. 


\section{Apêndice D}

\section{Demonstrações dos resultados do capítulo 4}

\section{Resultados da seção 4.1.1}

Prova da Proposição 4.1.5: Começamos lembrando que $\left(x_{0}, y_{0}\right)$ é um ponto de sela de $h$ se o gradiente $\left.\nabla h(x, y)\right|_{\left(x_{0}, y_{0}\right)}=\left.\left(\frac{\partial h}{\partial x}(x, y), \frac{\partial h}{\partial y}(x, y)\right)\right|_{\left(x_{0}, y_{0}\right)}$ for nulo e se a matriz hessiana

$$
\left.\nabla^{2} h(x, y)\right|_{\left(x_{0}, y_{0}\right)}=\left.\left(\begin{array}{cc}
\frac{\partial^{2} h}{\partial x^{2}}(x, y) & \frac{\partial^{2} h}{\partial x \partial y}(x, y) \\
\frac{\partial^{2} h}{\partial x \partial y}(x, y) & \frac{\partial^{2} h}{\partial y^{2}}(x, y)
\end{array}\right)\right|_{\left(x_{0}, y_{0}\right)}
$$

for indefinida. Como $h(x, y)=\exp \left(\lambda_{0}+\lambda_{10} x+\lambda_{01} y+\lambda_{11} x y\right)$ então obtemos que

$$
\left.\nabla h(x, y)\right|_{\left(x_{0}, y_{0}\right)}=\left(\lambda_{10}+\lambda_{11} y_{0}, \lambda_{01}+\lambda_{11} x_{0}\right)
$$

e que

$$
\left.\nabla^{2} h(x, y)\right|_{\left(x_{0}, y_{0}\right)}=h\left(x_{0}, y_{0}\right)\left(\begin{array}{cc}
0 & \lambda_{11} \\
\lambda_{11} & 0
\end{array}\right)
$$

Logo, $\left(x_{0}, y_{0}\right)=\left(-\frac{\lambda_{01}}{\lambda_{11}},-\frac{\lambda_{10}}{\lambda_{11}}\right)$ é ponto de sela de $h$. Portanto, somente precisamos provar que $\left.\left(x_{0}, y_{0}\right) \in\right] a, b[\times] a, b[$.

Se $f_{X}$ e $g_{Y}$ possuem um ponto mínimo em $x_{1}$ e $y_{1}$ respectivamente, então, pela Proposição 3.2 .5 , temos que

$$
L\left(x_{1}, \lambda_{01} ; \lambda_{11}\right)=\lambda_{10} \quad \text { e } \quad L\left(y_{1}, \lambda_{10} ; \lambda_{11}\right)=\lambda_{01}
$$


Pela definição da função $L$ obtemos

$$
\psi\left(x_{1} \lambda_{11}+\lambda_{01}\right)+a=-\frac{\lambda_{10}}{\lambda_{11}} \quad \text { e } \quad \psi\left(y_{1} \lambda_{11}+\lambda_{10}\right)+a=-\frac{\lambda_{01}}{\lambda_{11}} .
$$

Como a função $\psi$ é limitada,

$$
0<\psi(z)<b-a \text { para todo } z \in \mathbb{R}
$$

em particular concluímos que $\left.-\frac{\lambda_{01}}{\lambda_{11}} \mathrm{e}-\frac{\lambda_{10}}{\lambda_{11}} \in\right] a, b[$.

Se $f_{X \mid Y}$ e $g_{Y \mid X}$ são constantes para algum $x$ e $y$, então, pela Proposição 4.1.1, temos que $x=-\frac{\lambda_{01}}{\lambda_{11}}$ e $y=-\frac{\lambda_{10}}{\lambda_{11}}$ estão no intervalo $] a, b[$.

\section{Resultados da seção 4.1.2}

Prova de Lema 4.1.7: Calculamos a derivada

$$
\frac{\partial f_{X \mid Y}}{\partial x}(x \mid y)=f_{X \mid Y}(x \mid y)\left(\lambda_{10}+\lambda_{11} y+2 \lambda_{20} x\right) .
$$

A expressão acima pode ser nula apenas para $x=-\frac{\left(\lambda_{10}+\lambda_{11} y\right)}{2 \lambda_{20}}$. Isto prova a unicidade do ponto crítico.

Prova da Proposição 4.1.8: Pela expressão

$$
\frac{\partial f_{X \mid Y}}{\partial x}(x \mid y)=f_{X \mid Y}(x \mid y)\left(\lambda_{10}+\lambda_{11} y+2 \lambda_{20} x\right),
$$

concluímos que $\frac{\partial f_{X \mid Y}}{\partial x}(x \mid y)>0$ se, e somente se, $\lambda_{10}+\lambda_{11} y+2 \lambda_{20} x>0$. Então, se $\lambda_{20}>0$,

$$
\min _{x \in[a, b]} \lambda_{10}+\lambda_{11} y+2 \lambda_{20} x=\lambda_{10}+\lambda_{11} y+2 \lambda_{20} a .
$$

Logo, $\lambda_{10}+\lambda_{11} y+2 \lambda_{20} a \geq 0$ implica que a derivada é positiva para todo $\left.x \in\right] a, b[$. Quando $\lambda_{20}<0$,

$$
\min _{x \in[a, b]} \lambda_{10}+\lambda_{11} y+2 \lambda_{20} x=\lambda_{10}+\lambda_{11} y+2 \lambda_{20} b .
$$

Então $\lambda_{10}+\lambda_{11} y+2 \lambda_{20} b \geq 0$ faz com que a derivada seja positiva. 
Equivalentemente provamos o caso decrescente, mas estudando o máximo de $\lambda_{10}+\lambda_{11} y+2 \lambda_{20} x$.

Prova do Lema 4.1.9: Precisamos demonstrar que o sistema de equações

$$
\left\{\begin{array}{l}
c=\frac{-\lambda_{10}-2 \lambda_{20} a}{\lambda_{11}} \\
d=\frac{-\lambda_{10}-2 \lambda_{20} b}{\lambda_{11}}
\end{array}\right.
$$

possui solução nas variáveis $\lambda_{10}, \lambda_{11}$ e $\lambda_{20}$. Isto é equivalente a ver que existem $\lambda_{10}, \lambda_{11}$ e $\lambda_{20}$ tais que

$$
\left\{\begin{array}{l}
\lambda_{11} \neq 0 \\
\lambda_{11} c=-\lambda_{10}-2 \lambda_{20} a \\
(c-d) \lambda_{11}=2 \lambda_{20}(b-a) .
\end{array}\right.
$$

É imediato ver que o sistema acima possui infinitas soluções para quaisquer valores de $c$ e $d$.

Prova da Proposição 4.1.10: Primeiramente assumimos que $f_{X \mid Y}(\cdot \mid y)$ é crescente, implicando que $\lambda_{10}+\lambda_{11} y+2 \lambda_{20} x>0$ para todo $\left.x \in\right] a, b\left[\right.$. Como $\lambda_{20}<0$, então $\min _{x \in[a, b]}\left(\lambda_{10}+\lambda_{11} y+2 \lambda_{20} x\right)^{2}=$ $\left(\lambda_{10}+\lambda_{11} y+2 \lambda_{20} b\right)^{2}$. Logo, se $-2 \lambda_{20} \leq\left(\lambda_{10}+\lambda_{11} y+2 \lambda_{20} b\right)^{2}$, então, pela equação (4.6), concluímos que $f_{X \mid Y}(\cdot \mid y)$ possui segunda derivada positiva, o que significa que é uma função convexa. Reciprocamente, se a segunda derivada em $x$ de $f_{X \mid Y}(x \mid y)$ for não negativa para todo $x \in] a, b\left[\right.$, então $\left(\lambda_{10}+\lambda_{11} y+2 \lambda_{20} x\right)^{2}+2 \lambda_{20} \geq 0$. Tomando o limite de $x$ tendendo para $b$ obtemos a desigualdade $\left(\lambda_{10}+\lambda_{11} y+2 \lambda_{20} b\right)^{2}+2 \lambda_{20} \geq 0$.

Similarmente obtemos que $\max _{x \in[a, b]}\left(\lambda_{10}+\lambda_{11} y+2 \lambda_{20} x\right)^{2}=\left(\lambda_{10}+\lambda_{11} y+2 \lambda_{20} a\right)^{2}$ e $-2 \lambda_{20} \geq\left(\lambda_{10}+\lambda_{11} y+2 \lambda_{20} a\right)^{2}$ implica segunda derivada negativa.

Agora consideramos $f_{X \mid Y}(\cdot \mid y)$ decrescente, então $\lambda_{10}+\lambda_{11} y+2 \lambda_{20} x<0$ para todo $x \in] a, b\left[\right.$. Para este caso $\min _{x \in[a, b]}\left(\lambda_{10}+\lambda_{11} y+2 \lambda_{20} x\right)^{2}=\left(\lambda_{10}+\lambda_{11} y+2 \lambda_{20} a\right)^{2}$ e $\max _{x \in[a, b]}\left(\lambda_{10}+\lambda_{11} y+2 \lambda_{20} x\right)^{2}=\left(\lambda_{10}+\lambda_{11} y+2 \lambda_{20} b\right)^{2}$. Concluímos a demonstração analogamente ao caso crescente. 
Prova do Corolário 4.1.11: A demonstração é conseqüência da continuidade da segunda derivada e da Proposição 4.1.10.

Raciocinando pelo absurdo, assumimos que existem $y_{1}$ e $y_{2}, a<y_{1}<y_{2}<b$ tais que $f_{X \mid Y}(\cdot \mid y)$ é côncava para $y \leq y_{1}$ e $f_{X \mid Y}(\cdot \mid y)$ é convexa para $y_{1}<y \leq y_{2}$, ou seja

$$
\left.a \leq y \leq y_{1} \Rightarrow\left(\lambda_{10}+\lambda_{11} y+2 \lambda_{20} x\right)^{2}+2 \lambda_{20} \leq 0, \quad \text { para todo } x \in\right] a, b[
$$

e

$$
\left.y_{1}<y \leq y_{2} \Rightarrow\left(\lambda_{10}+\lambda_{11} y+2 \lambda_{20} x\right)^{2}+2 \lambda_{20} \geq 0, \quad \text { para todo } x \in\right] a, b[.
$$

Tomando o limite nas desigualdades acima para quando $y$ tende para $y_{1}$ pela direita e pela esquerda, concluímos que

$$
\left.\left(\lambda_{10}+\lambda_{11} y_{1}+2 \lambda_{20} x\right)^{2}+2 \lambda_{20}=0 \quad \text { para todo } x \in\right] a, b[.
$$

A relação anterior é absurda pois $\lambda_{20} \neq 0$. Portanto está demonstrado o corolário.

Prova da Proposição 4.1.12: Começamos calculando o gradiente da densidade $h(x, y)=\exp \left(\lambda_{0}+\lambda_{10} x+\lambda_{01} y+\lambda_{11} x y+\lambda_{20} x^{2}\right)$,

$$
\nabla h(x, y)=h(x, y)\left(\lambda_{10}+\lambda_{11} y+2 \lambda_{20} x, \lambda_{01}+\lambda_{11} x\right) .
$$

Logo, é imediato ver que existe um único ponto $\left(x_{0}, y_{0}\right)$ que anula o gradiente, sendo $\left(x_{0}, y_{0}\right)=\left(-\frac{\lambda_{01}}{\lambda_{11}}, \frac{2 \lambda_{20} \lambda_{01}}{\lambda_{11}^{2}}-\frac{\lambda_{10}}{\lambda_{11}}\right)$.

Por outro lado, a matriz hessiana no ponto crítico $\left(x_{0}, y_{0}\right)$ é

$$
\left.\nabla^{2} h(x, y)\right|_{\left(x_{0}, y_{0}\right)}=h\left(x_{0}, y_{0}\right)\left(\begin{array}{cc}
2 \lambda_{20} & \lambda_{11} \\
\lambda_{11} & 0
\end{array}\right) \text {. }
$$

A matriz anterior é indefinida e portanto o ponto crítico é ponto de sela. 


\section{Resultados da seção 4.1 .3}

Prova da Proposição 4.1.13: A demonstração é conseqüência da expressão do gradiente dada a seguir

$$
\nabla h(x, y)=h(x, y)\left(\lambda_{10}+\lambda_{11} y+2 \lambda_{20} x, \lambda_{01}+\lambda_{11} x+2 \lambda_{02} y\right) .
$$

Se $4 \lambda_{02} \lambda_{20}-\lambda_{11}^{2} \neq 0$, então existe uma única solução do sistema $\left(\lambda_{11} y+2 \lambda_{20} x, \lambda_{11} x+2 \lambda_{02} y\right)$, e portanto um único ponto crítico. Pelo contrário, se $4 \lambda_{02} \lambda_{20}-\lambda_{11}^{2}=0$, pode ser visto que não existe ponto crítico.

Prova da Proposição 4.1.14: Para determinar se o ponto crítico de $h$ é um ponto de sela, um ponto minimizador ou um ponto maximizador, temos que ver se a matriz

$$
\left.\nabla^{2} h(x, y)\right|_{\left(x_{0}, y_{0}\right)}=h\left(x_{0}, y_{0}\right)\left(\begin{array}{cc}
2 \lambda_{20} & \lambda_{11} \\
\lambda_{11} & 2 \lambda_{02}
\end{array}\right)
$$

é indefinida, definida positiva ou definida negativa, respectivamente. Como a matriz $\nabla^{2} h$ é simétrica, ver que é definida positiva é equivalente a ver que os autovalores são positivos, analogamente, definida negativa é equivalente a ter autovalores negativos e a propriedade de indefinida é equivalente a ter autovalores com diferente sinal. Uma exposição mais detalhada de tais equivalências é apresentada, por exemplo, em Luenberger (1989).

Os autovalores da matriz são

$$
\begin{aligned}
& \alpha_{1}=\lambda_{20}+\lambda_{02}+\sqrt{\lambda_{11}^{2}+\left(\lambda_{20}-\lambda_{02}\right)^{2}}, \\
& \alpha_{2}=\lambda_{20}+\lambda_{02}-\sqrt{\lambda_{11}^{2}+\left(\lambda_{20}-\lambda_{02}\right)^{2}} .
\end{aligned}
$$

Se $\lambda_{20}+\lambda_{02}=0$ é imediato ver que $\alpha_{1}>0>\alpha_{2}$, ou seja, a matriz é indefinida.

Se $\lambda_{11}^{2}-4 \lambda_{02} \lambda_{20}<0<\lambda_{20}+\lambda_{02}$, então

$$
\begin{aligned}
\lambda_{11}^{2}-2 \lambda_{20} \lambda_{02} & <2 \lambda_{20} \lambda_{02} \\
\lambda_{11}^{2}+\left(\lambda_{20}-\lambda_{02}\right)^{2} & <\left(\lambda_{20}+\lambda_{02}\right)^{2} \\
\sqrt{\lambda_{11}^{2}+\left(\lambda_{20}-\lambda_{02}\right)^{2}} & <\lambda_{20}+\lambda_{02},
\end{aligned}
$$


$\log \alpha_{2}>0$. O autovalor $\alpha_{1}$ resulta ser positivo por ser soma de dois termos positivos. Portanto a matriz é definida positiva.

Se $\lambda_{11}^{2}-4 \lambda_{02} \lambda_{20}<0$ e $0>\lambda_{20}+\lambda_{02}$, então $\alpha_{2}<0$ por ser soma de dois valores negativos. Por outro lado, trabalhando analogamente ao caso anterior, obtemos que

$$
\begin{aligned}
\lambda_{11}^{2}+\left(\lambda_{20}-\lambda_{02}\right)^{2} & <\left(\lambda_{20}+\lambda_{02}\right)^{2} \\
\sqrt{\lambda_{11}^{2}+\left(\lambda_{20}-\lambda_{02}\right)^{2}} & <\left|\lambda_{20}+\lambda_{02}\right|=-\left(\lambda_{20}+\lambda_{02}\right),
\end{aligned}
$$

ou seja, $\alpha_{1}<0$. A matriz é definida negativa.

Finalmente, se $\lambda_{11}^{2}-4 \lambda_{02} \lambda_{20}>0$, então

$$
\begin{aligned}
\lambda_{11}^{2}+\left(\lambda_{20}-\lambda_{02}\right)^{2} & >\left(\lambda_{20}+\lambda_{02}\right)^{2} \\
\sqrt{\lambda_{11}^{2}+\left(\lambda_{20}-\lambda_{02}\right)^{2}} & >\left|\lambda_{20}+\lambda_{02}\right| .
\end{aligned}
$$

Como $\left|\lambda_{20}+\lambda_{02}\right| \geq \pm\left(\lambda_{20}+\lambda_{02}\right)$, conluímos que $\alpha_{2}<0$ e que $\alpha_{1}>0$, ou seja, a matriz é indefinida.

\section{Resultados da seção 4.2}

Prova de Proposição 4.2.4: Começamos calculando a primeira e segunda derivada de $f_{X \mid Y}(x \mid y)$ na variável $x$,

$$
\frac{\partial f_{X \mid Y}}{\partial x}(x \mid y)=f_{X \mid Y}(x \mid y)\left(\lambda_{10}+\lambda_{11} y+2 \lambda_{21} y x\right)
$$

e

$$
\frac{\partial^{2} f_{X \mid Y}}{\partial x^{2}}(x \mid y)=f_{X \mid Y}(x \mid y)\left(\left(\lambda_{10}+\lambda_{11} y+2 \lambda_{21} y x\right)^{2}+2 \lambda_{21} y\right) .
$$

Da expressão da primeira derivada resultam imediatos os itens 1, 2 (a), 2 (b), 3 (a) e 3 (c). Acerca dos resultados dos pontos críticos, também da primeira derivada vemos que o único ponto crítico de $f_{X \mid Y}(\cdot \mid y)$ é $x_{0}=\frac{-\lambda_{10}-\lambda_{11} y}{2 \lambda_{21}}$. A segunda derivada em $x_{0}$ é

$$
\left.\frac{\partial^{2} f_{X \mid Y}}{\partial x^{2}}(x \mid y)\right|_{x=x_{0}}=2 f_{X \mid Y}\left(x_{0} \mid y\right) \lambda_{21} y
$$


Logo, se $\lambda_{21} y>0$, então o ponto crítico é um minimizador. Se, ao contrário, $\lambda_{21} y<0$, então $x_{0}$ é um minimizador. Concluímos assim a demonstração.

Prova da Proposição 4.2.10: O gradiente de $h$ está dado por

$$
\nabla h(x, y)=h(x, y)\left(\lambda_{10}+\lambda_{11} y+2 \lambda_{21} x y, \lambda_{01}+\lambda_{11} x+\lambda_{21} x^{2}\right) .
$$

Logo, existe solução para $\nabla h=(0,0)$ se $\lambda_{11}^{2}-4 \lambda_{21} \lambda_{01}>0$. Nesse caso são duas soluções,

$$
\begin{aligned}
& \left(x_{1}, y_{1}\right)=\left(\frac{-\lambda_{11}+\sqrt{\lambda_{11}^{2}-4 \lambda_{21} \lambda_{01}}}{2 \lambda_{21}},-\frac{\lambda_{10} \sqrt{\lambda_{11}^{2}-4 \lambda_{21} \lambda_{01}}}{\lambda_{11}^{2}-4 \lambda_{21} \lambda_{01}}\right), \\
& \left(x_{2}, y_{2}\right)=\left(\frac{-\lambda_{11}-\sqrt{\lambda_{11}^{2}-4 \lambda_{21} \lambda_{01}}}{2 \lambda_{21}}, \frac{\lambda_{10} \sqrt{\lambda_{11}^{2}-4 \lambda_{21} \lambda_{01}}}{\lambda_{11}^{2}-4 \lambda_{21} \lambda_{01}}\right) .
\end{aligned}
$$

Para ver que os pontos críticos são pontos de sela, basta ver que a matriz hessiana em tais pontos é indefinida. Sendo

$$
\left.\nabla^{2} h(x, y)\right|_{\left(x_{1}, y_{1}\right)}=h\left(x_{1}, y_{1}\right)\left(\begin{array}{cc}
2 \lambda_{21} y_{1} & \lambda_{11}+2 \lambda_{21} x_{1} \\
\lambda_{11}+2 \lambda_{21} x_{1} & 0
\end{array}\right)
$$

e

$$
\left.\nabla^{2} h(x, y)\right|_{\left(x_{2}, y_{2}\right)}=h\left(x_{2}, y_{2}\right)\left(\begin{array}{cc}
2 \lambda_{21} y_{2} & \lambda_{11}+2 \lambda_{21} x_{2} \\
\lambda_{11}+2 \lambda_{21} x_{2} & 0
\end{array}\right),
$$

resulta imediato ver que são matrizes indefinidas. Portanto fica provada a proposição. 